\title{
MINIMUM ++
}




\section{Minimum ++}

a 120 point thesis submitted to the Victoria University of Wellington in partial fulfilment of the requirements for the degree of Masters of Architecture (Professional)

\section{Lu Cheng}

Victoria University of Wellington, 2015

School of Architecture

Word Count: 10,412 


\section{ACKNOWLEDGMENTS}

I am using this opportunity to express my gratitude to everyone who supported me throughout the course of Master of Architecture (Professional). I am thankful for their aspiring guidance, invaluably constructive criticism and friendly advice during the design research thesis work. I am sincerely grateful to them for sharing their truthful and illuminating views on a number of issues related to the project.

I would like to express my special thanks of the gratitude to my supervisors, Mark Southcombe and Tane Moleta, who guided me through the year of work.

I would also like to thank Mike Cole from Archaus Architects, Bernadette Chong from Te Puni Village, and all the people who provided me with the facilities and information being required for my research.

At last but not the least I wish to thank my parents for their undivided support and interest who inspired me and encouraged me to pursue my own path, without whom I would be unable to complete my master thesis. 


\section{ABSTRACT}

Living quality and experience in medium or higher density student housing in Wellington is suffering from the inconsiderate internal spaces and underutilised common areas. The aim of this design-led research thesis is to provide design solutions that would be able to improve the quality of life in local student housing in architectural aspects. Several precedents were documented and analysed in each design phase to state the design directions and goals. Findings from each proposed design scheme was taken as base to expand and develop the next iteration. The three design phases have covered iterations of different project scales. Started from the designs of individual units to a block-wide student village, the iterations have addressed the research question by suggesting three design strategies, which were hyper efficiency, transitional shared space and blurred boundary. The design proposals illustrated how these strategies would be applied in different scales and how they would make positive impacts in terms of improving the quality of life in compact living environment. 


\section{TABLE OF CONTENTS}

\begin{tabular}{|c|c|}
\hline Acknowledgments & ii \\
\hline Abstract & iii \\
\hline List of Figures & $v$ \\
\hline 1. INTRODUCTION & 6 \\
\hline 1.1 SCOPE OF RESEARCH & 8 \\
\hline 2. METHODS \& STRUCTURE. & 9 \\
\hline 3. COMPACT HOUSING CASE STUUDIES & 11 \\
\hline 3.1 INTERNATIONAL CASE STUDIES: INDIVIDUAL UNITS & 12 \\
\hline 3.1.1 The Egg House & 12 \\
\hline 3.1.2 The Container House & 14 \\
\hline 3.1.3 The Cube Project QB1 & 16 \\
\hline 3.1.4 The Artist Studio & 18 \\
\hline 3.2 LOCAL CASE STUDIES: APARTMENT UNITS & 20 \\
\hline 3.2.1 DUEL on Vivian & 20 \\
\hline 3.2.2 SOHO Apartments & 22 \\
\hline
\end{tabular}

3.2.3 TE PUNI Village
3.3 FINDINGS AND REFLECTIONS
4. DESIGN PHASE I:
HYPER EFFICIENCY
4.1 COMPACT SINGLE UNIT: MAXIMISING SPACE
4.1.1 Space Transforming: Multi-Functional Components
4.1.2 Space Defining: Separation and Privacy
4.2 COMPACT TWO-BEDROOM UNIT: EXPANDING SPACE
4.3 FINDINGS AND REFLECTIONS
5. DESITIGN PHASE II:
SHARED SPACE
5.1 COMPA................................................
5.1.1 Site Analysis
5.1.2 Public Sharing: Introducing Community
5.2 SHARED HOUSE: POSITIVE SHARING SPACE

5.2.1 New Precedents: Japanese Share House $\quad 54$ 5.2.2 Positive Sharing: Internal Shared Space 56 5.3 SHARED HOUSE II: TRANSITIONAL SPACE 58 5.3.1 Private within Public: Continuous Circulation Loop 58 5.4 FINDINGS AND REFLECTIONS

\section{DESIGN PHASE III:}

BLURRING THE BOUNDARY

66

6.1 TE ARO VILLAGE: SHARING IN PUBLIC

6.1.1 Site Extension

6.1.2 Recessed Boundary

6.1.3 Negative Space

6.1.4 Design Proposal

6.2 FINDINGS AND REFLECTIONS

7. CONCLUSION

Bibliography
58

67

68

70

72

74

91

92

94 


\section{LIST OF FIGURES}

Figure 1. Tomlinson, S. (2013, February 22). Hong Kong's human battery hens. Daily Mail Online. Retrieved from: http:// www.dailymail.co.uk/news/article-2282764/Hong-Kongshuman-battery-hens-Claustrophobic-images-slum-familiessqueeze-lives-tiniest-apartments.htm

Figure 3. Ruan, Y. (2011, February 20). Construction of the Egg House [Web log message]. Retrieved from: http://www. ruanyifeng.com/blog/2011/02/building_an_egg-shaped_house. html

Figure 5-7. Huanglong. (2013). How's a $7 \mathrm{~m}^{2}$ house look like? Retrieved from: http://cq.cqnews.net/html/2013-04/10/ content_25497636.htm

Figure 9-11. The Cube Project. (2009). QB1: a history in pictures. Retrieved from: http://www.cubeproject.org.uk/gallery

Figure 13-16. IGNANT. (2014). Artist studio by Raanan Stern. Retrieved from: http://www.ignant.de/2014/03/17/artiststudio-by-raanan-stern/

Figure 18-19. Klauster Properties Limited. (2010). Furnished Studio Apartment in Vivian St, Wellington City. Retrieved from: http://klausterproperties.info/rentals/142vst.htm

Figure 21. Blair, 0. (2012). Spatial Urban Digital Intensified Radical Design Research Project. Retrieved from: http://www. spatialdesign.info/obr/soho-epiphyte/\#

Figure 23-25. ArchDaily. (2011). Te Puni Village / Architectus. Retrieved from: http://www.archdaily.com/140692/te-punivillage-architectus/

Figure 27. ArchDaily (2013). Studentboende: Student Unit/ Tengbom. Retrieved from: http://www.archdaily.com/430047/ studentboende-student-unit-tengbom/

Figure 48. Griffiths, A. (2013, August). Share House LT Josai by Naruse Inokuma Architects. dezeen magazine. Retrieved from http://www.dezeen.com/2013/08/29/share-house-by-naruseinokuma-architects/ 


\section{INTRODUCTION}

"energy-efficiency is something most people are learning to get their minds around, the idea that we might be able to live with both less consumption and less built space is striking some as a reasonable corollary to the conversation about using and creating energy wisely" (p. 3).

---- Richardson (2011)

Quality of life in medium or higher density housing has certainly been one of the enduring issues of the contemporary built environment. Many compact living solutions have been experimented with, especially in countries with overwhelming populations, but most provide a relatively low living standard. This thesis was motivated by a photo of a typical compact apartment in Hong Kong, where all the families' activities were being squeezed in a tiny space. Although New Zealand or Wellington more specifically is not as populated as Hong Kong, there are still some issues that

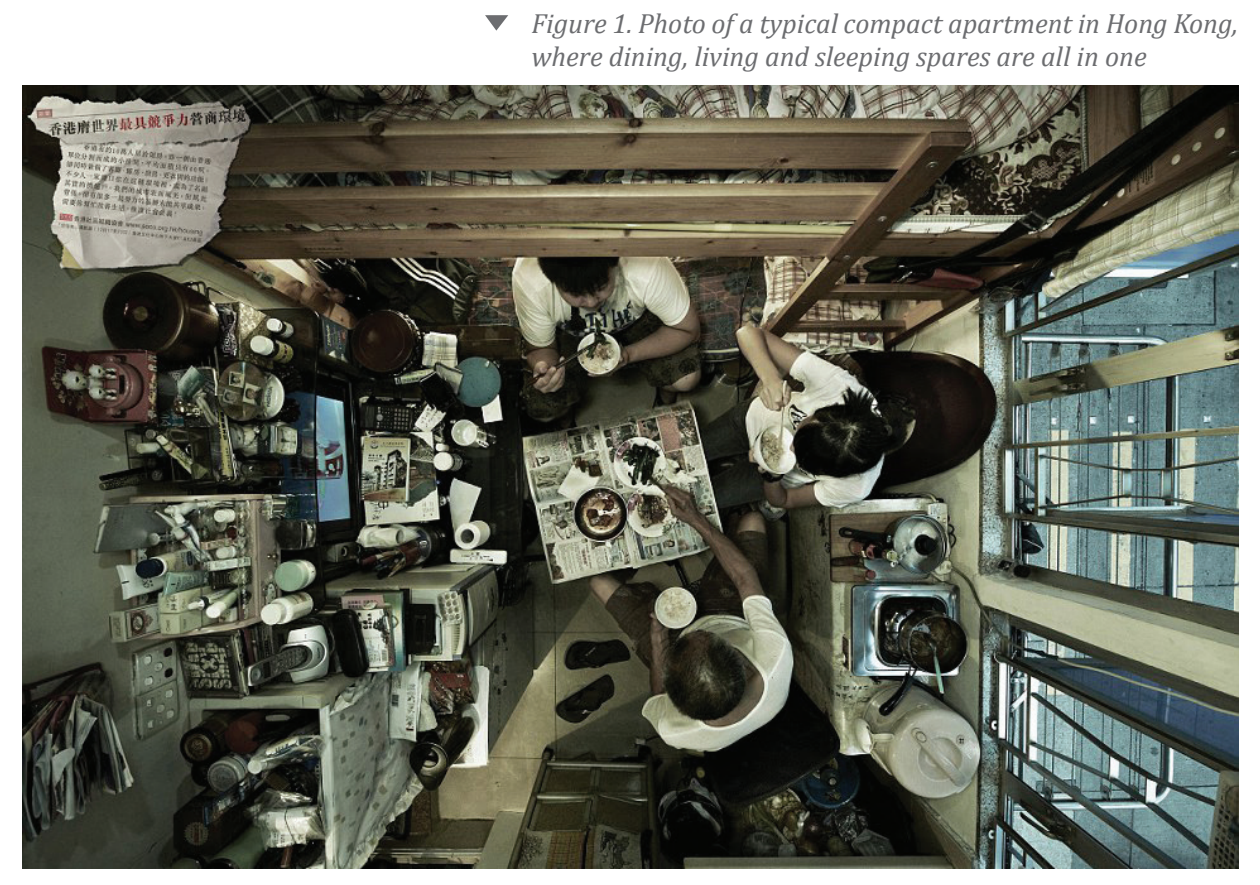


can be seen in the densely built environment, especially in inner city student housing or apartments targeted at students. Problems such as unfriendly entrances, inefficient spaces and underutilised common areas have had a negative impact on student living experience in Central Wellington. In order to change and improve the current situation, this design thesis has investigated quality compact living solutions of different scales, from individual housing to a block-wide urban student village. The covered design investigations aimed to address the question: "how can quality of life in medium or higher density student housing in Wellington be improved?"
Spatial efficiency is certainly one of the strategies that will make a positive impact in terms of quality of life in minimum dwellings. In the book 'Small Moves: architectural strategies for the unit in social housing', Andrea (2012) introduced a concept of hyper efficiency:

"hyper efficiency is a strategy that seeks to maximise the use and functionality of space inside the unit. In a hyper efficient unit, no space is unused or under-use; all possible space has dual, triple, or even quadruple purposes" (p. 54).

Other than the internal efficiency, quality of the surrounded external space is another important factor that affects the living standards. Suggested by Goodchild (1997) in his book 'Housing and the Urban Environment':

"most discussion of quality in housing design concentrated on its socio-cultural aspects. They focus on how people experience the environment around them; how they interact with that environment; and how they judge its suitability in relation to their daily routines and their expectations for the future" (p.32).

The initial research components of this thesis began by studying four international precedents and three local ones, in order to analyse and critique the current local situation and learn from overseas examples of possible solutions. 
The research then moved through three design phases which investigated and proposed design solutions with positive impact on improving the quality of life in high density student housing in Wellington. Design Phase I has tested the possible ways of achieving internal hyper efficiency in individual houses as a way to extend the amenity within compact sutdent living. Design Phase II looked at how the idea of hyper efficiency could be extended into common areas within an apartment as shared spaces. The final design phase carried the shared space concept through and extended it to external aspects. This phase tested the surrounded shared public space at a larger scale. All proposed solutions were developed based on the findings of the previous phases and aguments by additional research.
They have covered the factors that affect quality of life in compact living environment at different scales, as well as suggested new typologies that could improve local compact student living standards.

\subsection{SCOPE OF RESEARCH}

As "quality of life" refers to many aspects, the scope of this thesis was focused on spatial efficiency and the balance between private and public spaces in medium or high density housing. The design iterations were targeted at internal layout variations, ways of utilising transformative spaces, as well as the significance of the "third space" shared in between. Other possible aspects that would affect quality of life such as construction methods including material uses and affordability were not covered in depth by this thesis. 


\section{METHODS \& STRUCTURE}

This is a design-led research thesis which has been developed through multiple iterations over three design phases. An initial overview and critique of the current situation of student targeted accommodations in Wellington stated the problem and the motivation of the research. Precedent studies were introduced from the beginning of each design phases and influenced the key design goals at each stage. The three phases have provided design solutions to improve living quality for different scales, which are individual unit, single apartment block and student village with a group of apartments. Each phase has been developed and expanded from the findings and reflections from the previous. The project has been designed specifically for a site and its context in Te Aro, Wellington, however the findings and design strategies are relevant to any inner city medium or higher density student housing projects throughout New Zealand. 


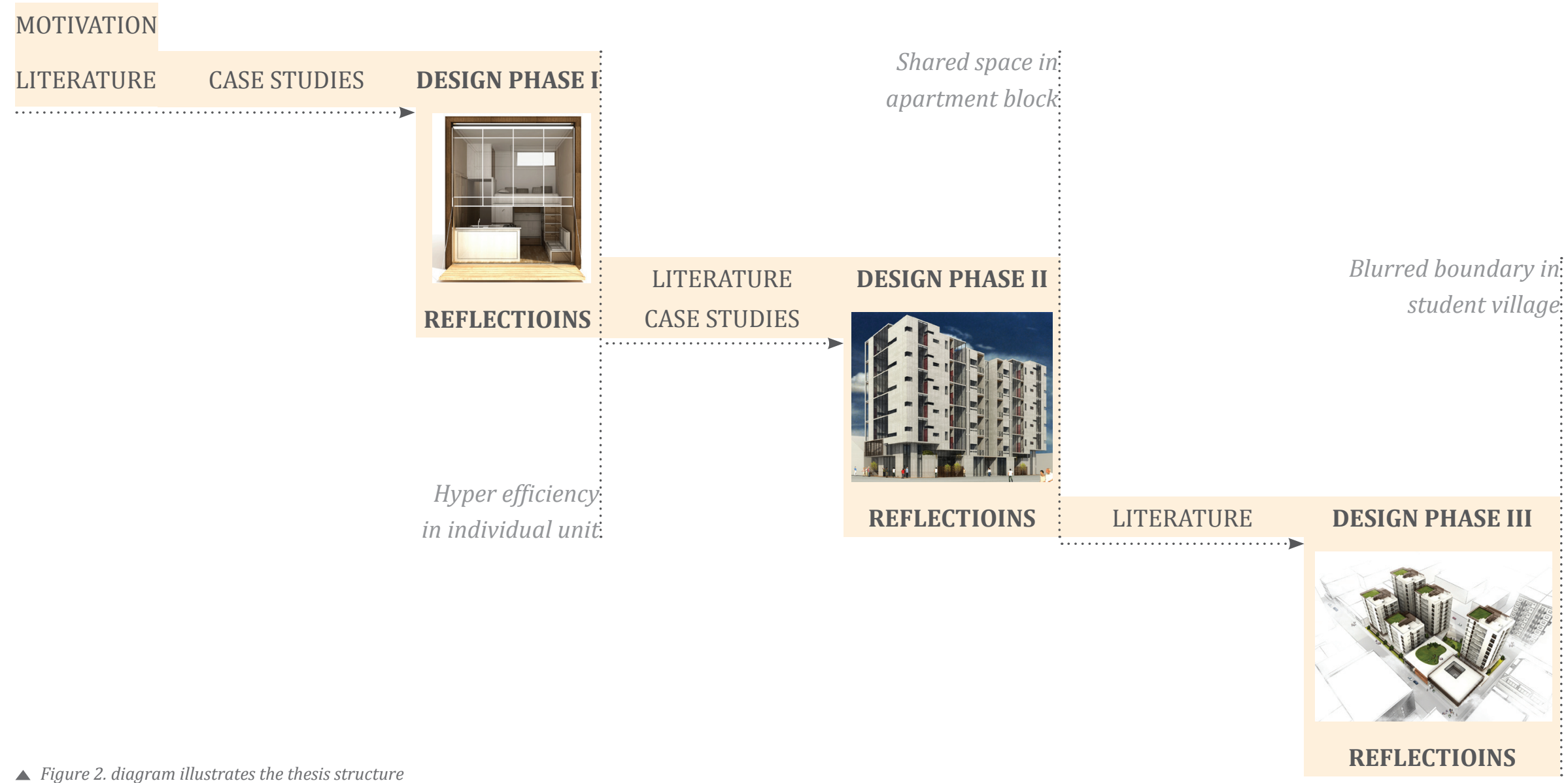




\section{COMPACT HOUSING CASE STUDIES}

In order to understand the current problems and solutions in compact living environments, investigation began by studying both international and local precedents. Four international, and three local precedents were documented of varying sizes and scales. Analysis of Wellington's problems and reflection on possible international solutions set the framework for the later design investigation. 


\subsection{INTERNATIONAL CASE STUDIES:}

\section{INDIVIDUAL UNITS}

\subsubsection{The Egg House by Haifei Dai}

\section{- Building area: $5 \mathrm{~m}^{2}$}

Floor area: $5 \mathrm{~m}^{2}$

- Ceiling height: $2.3 \mathrm{~m}$ (wheels $0.4 \mathrm{~m}+$ internal $1.9 \mathrm{~m}$ )

The egg house ${ }^{[1]}$ was designed and constructed by a Chinese architectural graduate, Haifei Dai, who found the rental was extremely high in Beijing and tried to look for more affordable living solutions for himself. The egg house was constructed of steel framing, screwed fixtures and covered by seed bags as cladding. The furniture was custom made to be fit and maximised the use of the tiny space. Mobility and affordability are two of the main strengths that achieved in the design of the egg house. With a construction cost of approximately $\$ 1,000$ USD, the egg house can be easily transported and settled anywhere on the street. In terms of living quality, the egg house is considered below the standard as it has no kitchen and toilet facilities. Safety is another concern as there is no protected boundaries to define the private space. Despite its limits in providing a quality living environment, the egg house is certainly a good example on how space can be used in minimum housing. With a five square meter internal area, it provides an efficient space which enough for one person to work and sleep in.

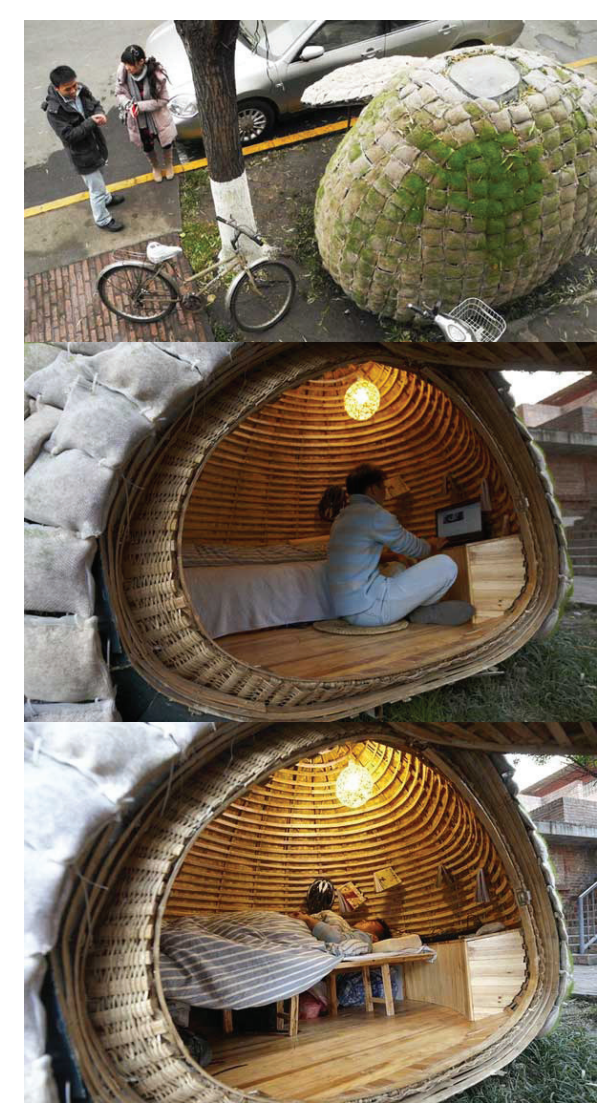

1 Ruan, Y. (2011, February 20). Construction of the Egg House. 


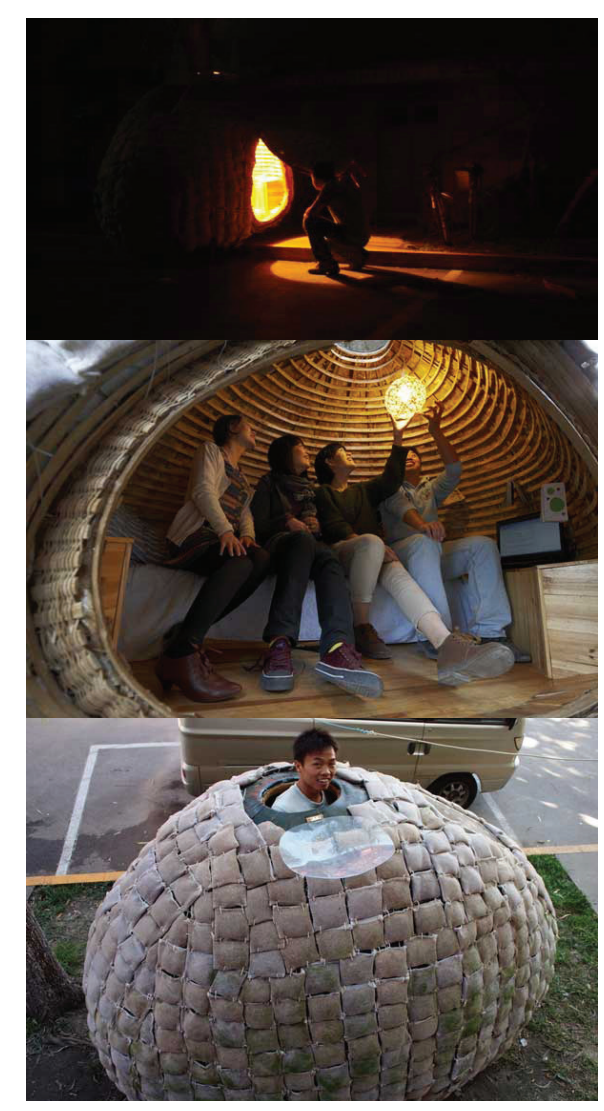

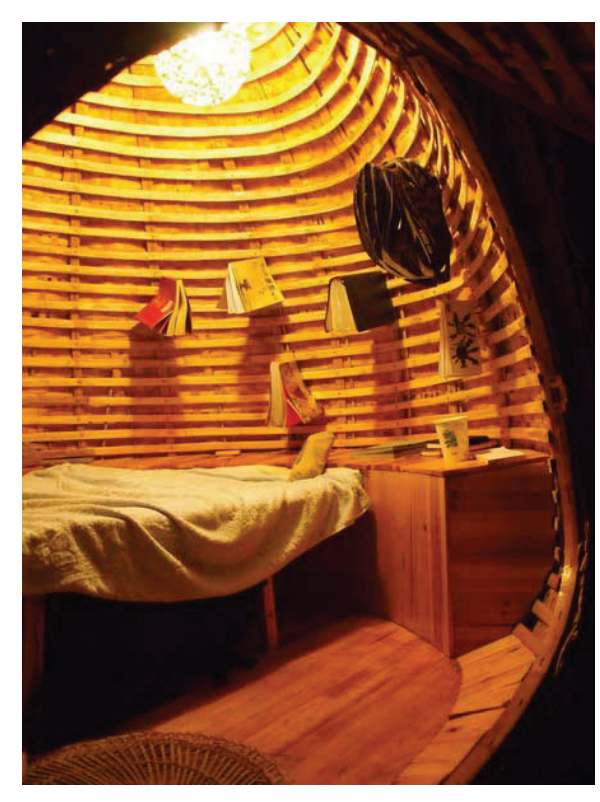

4 Figure 3. Photos of the egg house shown how it was being used in different time of a day

- Figure 4. Plan and section that

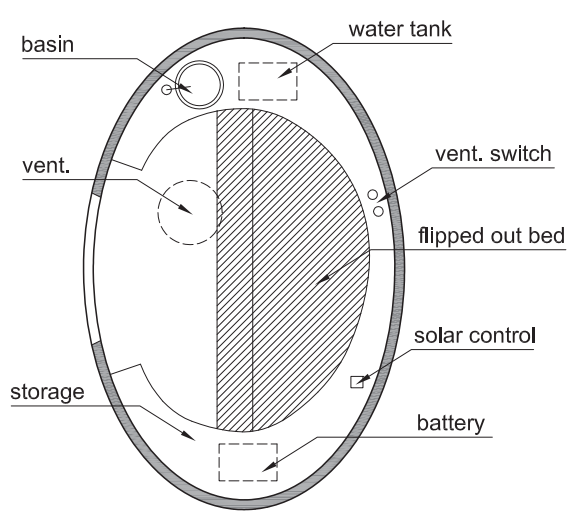

grass seed
bag cladding

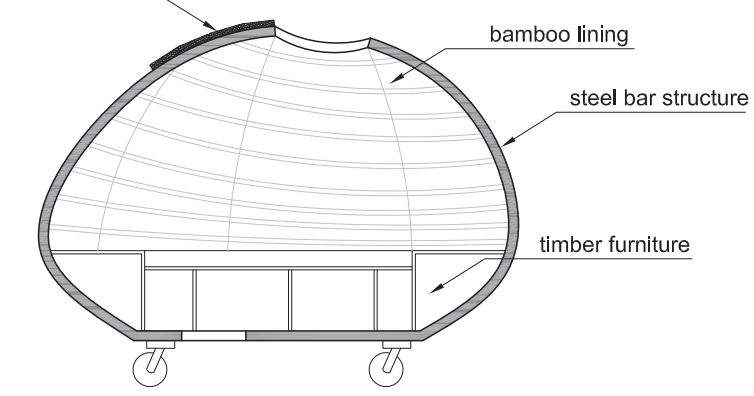


3.1.2 The Container House by Chongqing University

- Building area: $7 \mathrm{~m}^{2}$

Floor area: $12 \mathrm{~m}^{2}$

- Ceiling height: $3.6 \mathrm{~m}$

The container house ${ }^{[1]}$ was designed and constructed by several students from the school of architecture, Chongqing University. Within a space of $3.3 \times 2.4$ meters, they have created a comfortable one bedroom mezzanine studio that includes a kitchen, bathroom and a transformative dining and study area. It was an experimental project which tried to address the overwhelming population and extremely expansive real estate market in the major cities of China. By creating space with double duty, the idea of spatial efficiency started to take place. In this case the dining table and book shelves were hidden in the raised floor platform, which allows the space to be transformed from a flat, visually spacious living area, into a dining and study space. This idea of double duty space has really provided a way of utilising space efficiently in compact living environments.

- Figure 5. Photo of transformative dining and living area with raised table and book shelves

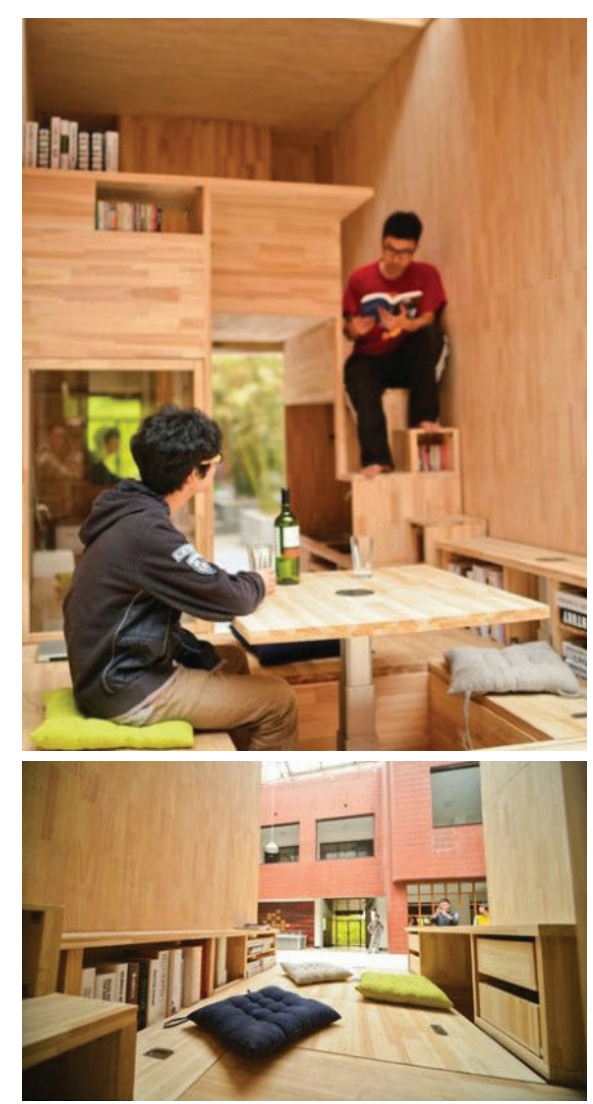

1 Huanglong. (2013). How's a $7 m^{2}$ house look like? 

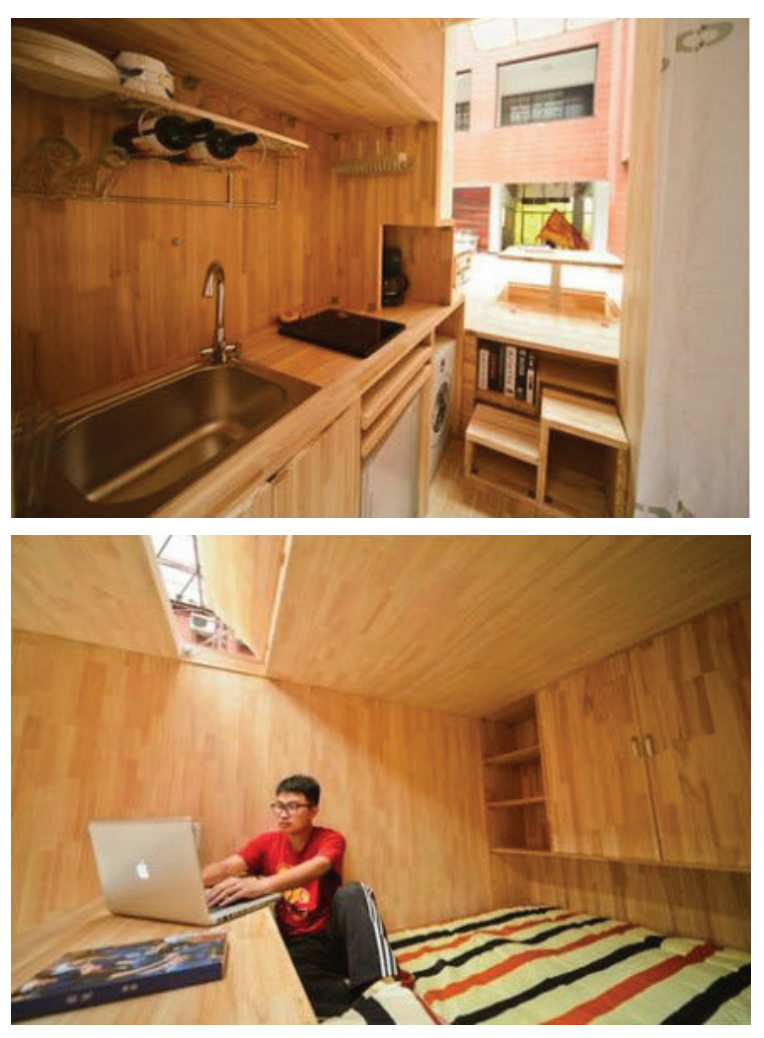

- Figure 6. Corridor in between kitchen and toilet

- Figure 7. Sleeping area on mezzanine level

- Figure 8. Container House floor plans
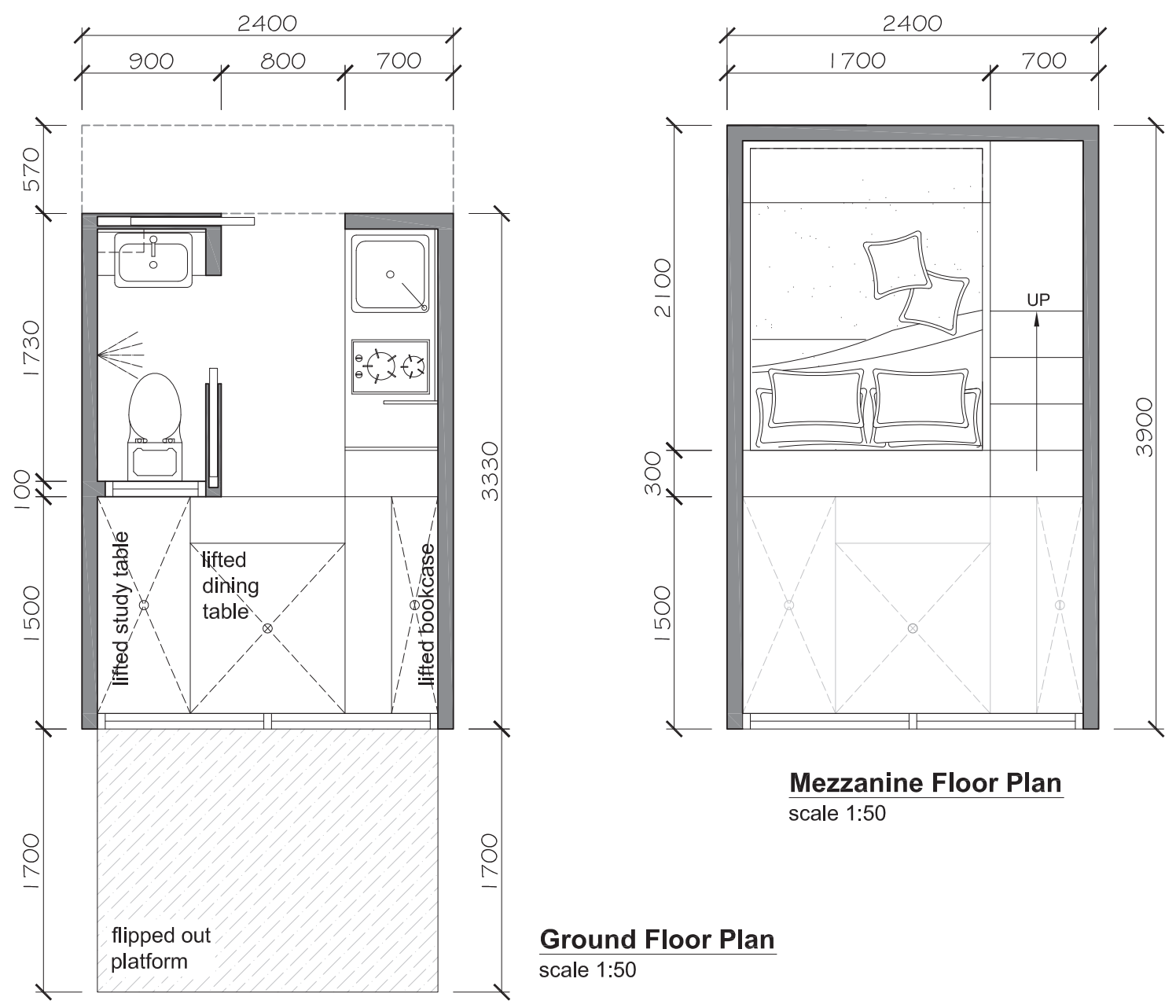

Mezzanine Floor Plan scale 1:50
Ground Floor Plan scale 1:50 
- Building area: $10 \mathrm{~m}^{2}$

- Floor area: $15 \mathrm{~m}^{2}$

- Ceiling height: $3 \mathrm{~m}$ ( $2 \mathrm{~m}$ on each level)

The Cube Project ${ }^{[1]}$ is a good example of quality minimum housing. Designed by Dr. Mike Page from the University of Herfordshire, the Cube Project is a studio unit based on $3 \times 3 \times 3$ metre cube. Including full facilities and sustainable features, the Cube presents a successful way of living in compact space. The internal space is divided into three overlapping split levels, each with a two metre ceiling height. Dr. Mike Page claimed that no space was wasted under such restricted ceiling height. A sliding table and separate toilet and shower allow the Cube to

1 The Cube Project. (2009). QB1: a history in pictures. efficiently occupy by a couple. Major achievement with the Cube, in comparison to the previous case studies is the separation of space by split levels. It draws lines between different functional areas to give a sense of separated rooms in tiny studio space. The cleverly designed sliding furniture provides a comfortable space for one person and enough space for two by rearranging its configuration. Windows are nicely planned on each level so the tiny space can be easily lit naturally.

- Figure 9. Photo of $3 m^{3}$ unit placed on site with an attached rooftop that contains sustainable solar heating system

- Figure 10. View from the staircase to ground floor living area with the sliding table
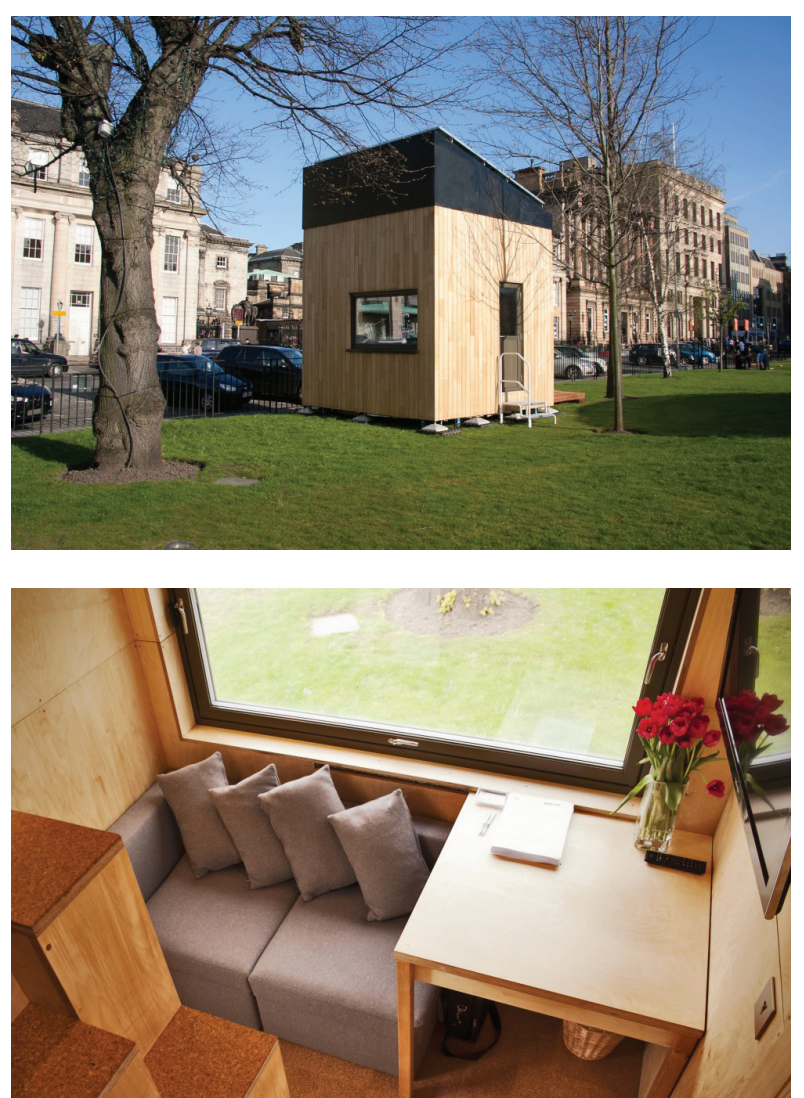


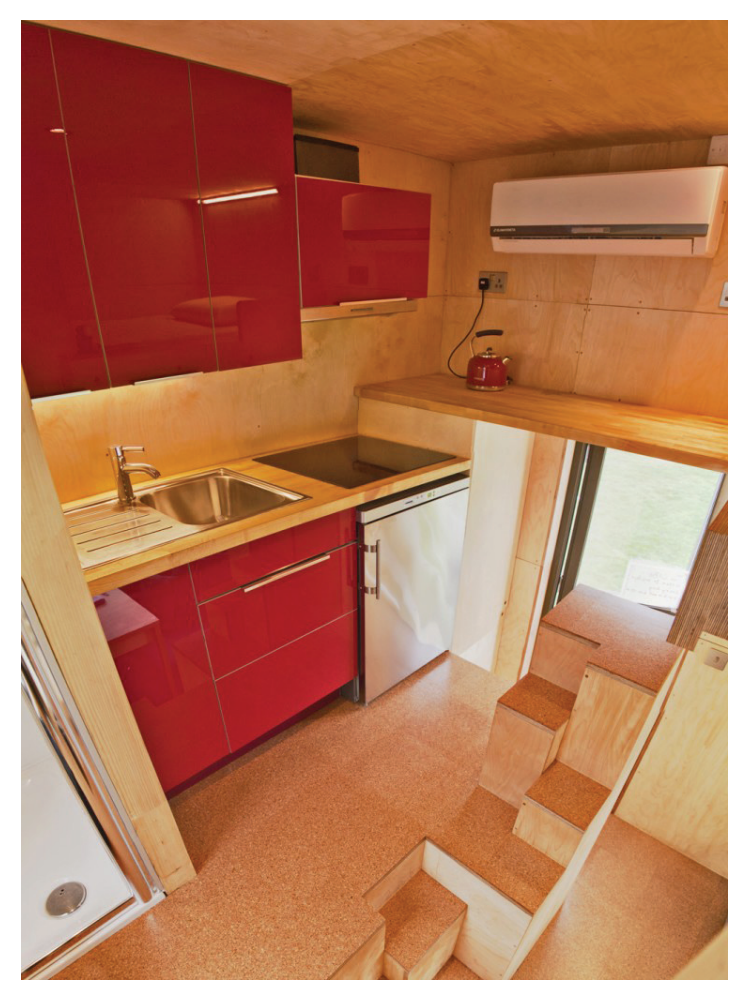

- Figure 11. View from staircase look into the kitchen on mezzanine level with visual opening to the main entrance

- Figure 12. The Cube floor plans

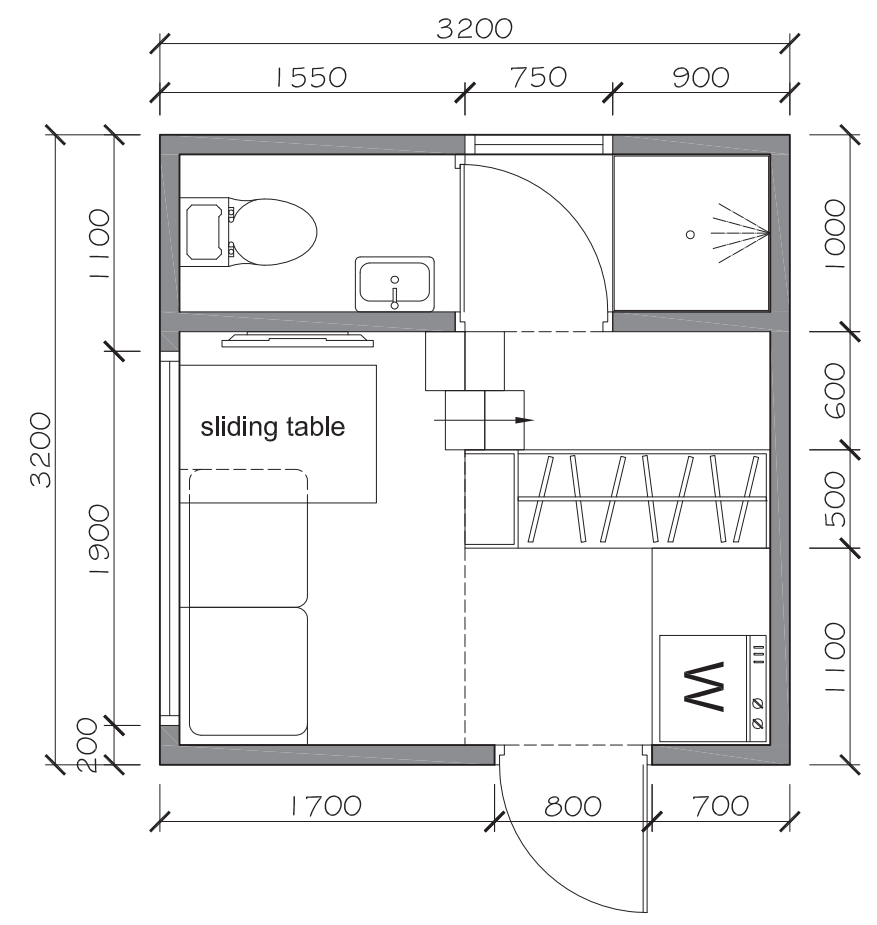

Ground Floor Plan scale 1:50

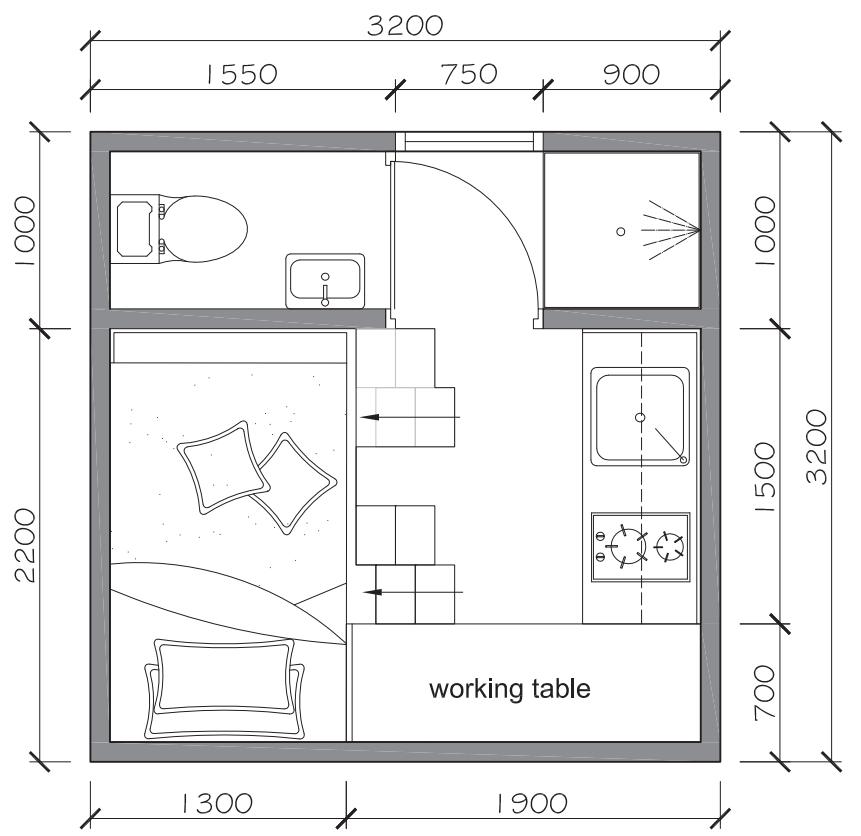

Mezzanine Floor Plan scale 1:50 


\subsubsection{The Artist Studio by Raanan Stern}

Building area: $18 \mathrm{~m}^{2}$

Floor area: $18 \mathrm{~m}^{2}$

Ceiling height: $2.6 \mathrm{~m}$

This artist studio ${ }^{[1]}$ was designed to fit the specific needs of an artist as a working and resting place or even a guest bedroom. Storage cabinets were clever planned as it was one of the crucial issues to keep the space tiny enough for resting. A fold out single bed is hidden behind a well-designed sliding panel that also acts as a peg board to display artworks. A large drafting table on wheels can be easily moved aside to provide more space for some large artworks to

1 IGNANT. (2014). Artist studio by Raanan Stern. be done on the floor. The main idea of the design of this studio was to provide spaces that could keep everything stored nicely and so achieve a sense of visual spacious in a tiny space.

- Figure 13. Well planned storage configuration that specifically suits the artist's needs

Figure 14. A single bed can be fold out from behind the sliding peg board to create a temporary sleeping area

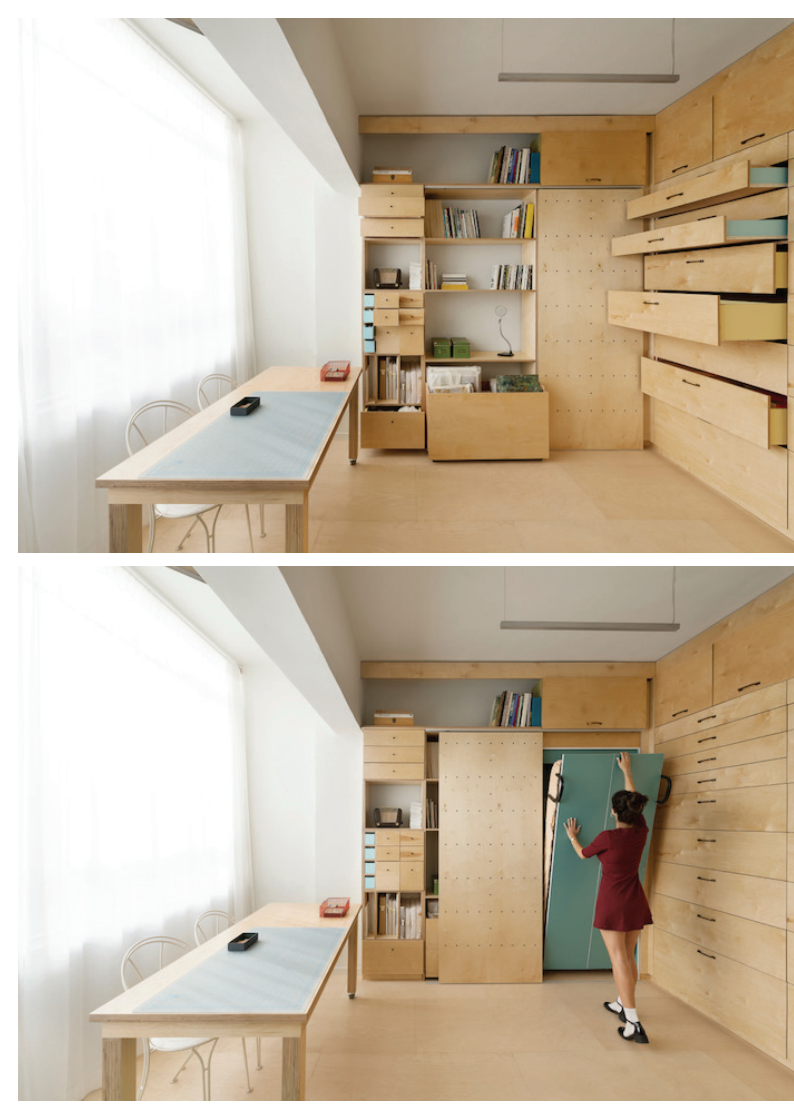




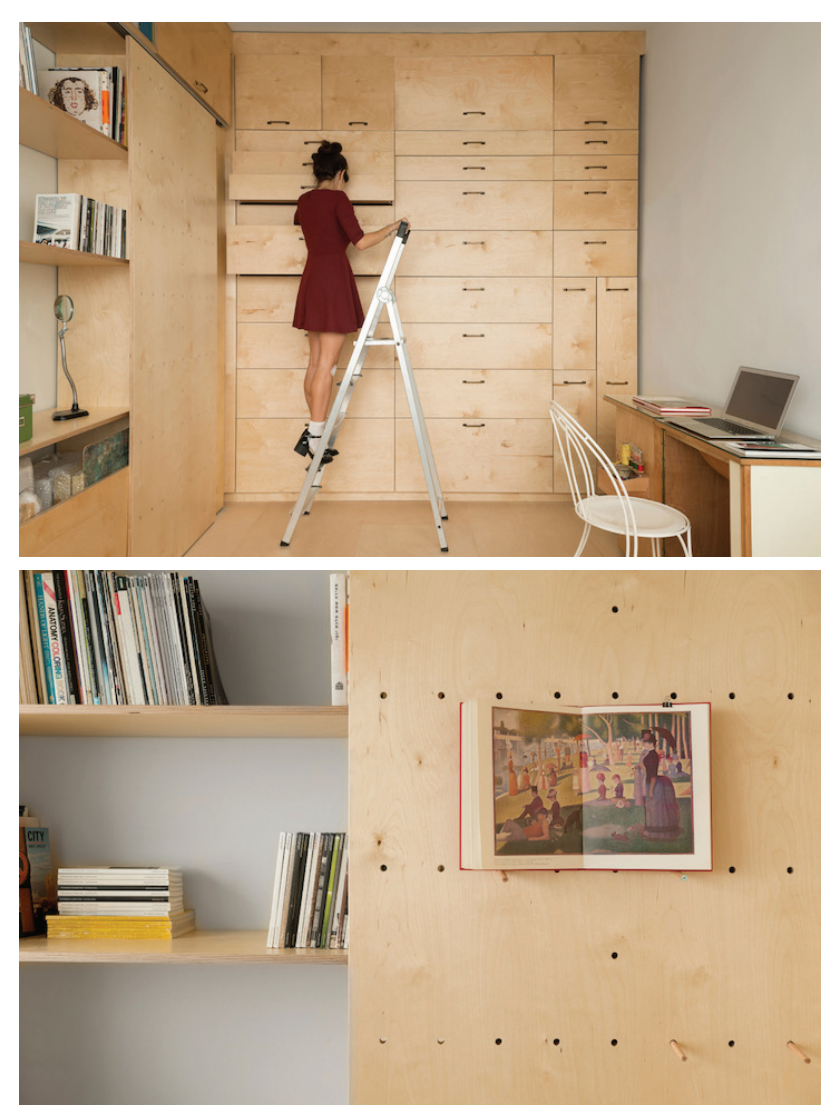

- Figure 15. Drawers are varying in sizes to suit the specific need of storing method

\ Figure 16. The multi-duty peg board serves as an artwork display board, as well as a sliding panel that covers either the display shelf or the fold out bed

- Figure 17. Artist Studio floor plan

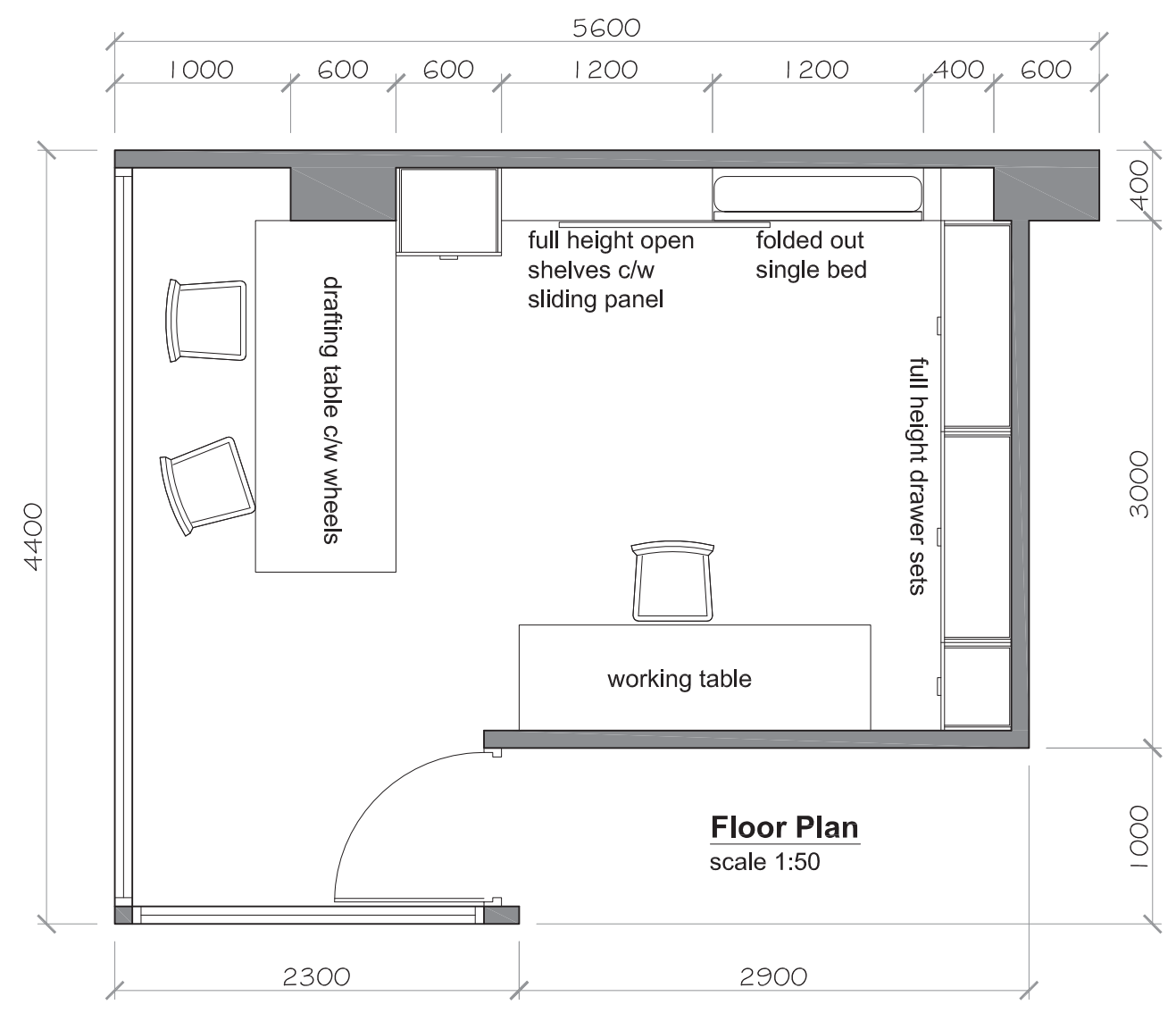




\subsection{LOCAL CASE STUDIES: APARTMENT UNITS}

\subsubsection{DUEL on Vivian}

\section{Floor area: $24.5 \mathrm{~m}^{2}$}

Balcony area: $3.6 \mathrm{~m}^{2}$

Ceiling height: $2.8 \mathrm{~m}$

One central Wellington apartment building targeting students is the DUEL apartments on Vivian Street, opposite Victoria University of Wellington Te Aro Campus. Unit types vary from studios to two bedroom apartments in this five storey building. In terms of quality living, all the rooms are fully furnished with private kitchens and bathrooms, and balconies overlooking the street. The floor plan shows that storage space in the studio unit is very limited in relation to its
$25 \mathrm{~m}^{2}$ floor area. Entering from a narrow corridor straight into the kitchen is another drawback on spatial experience. Due to the fact that the bed is the focus of the room, it makes the unit look no difference to a conventional hotel room.

- Figure 18. DUEL on Vivian street view

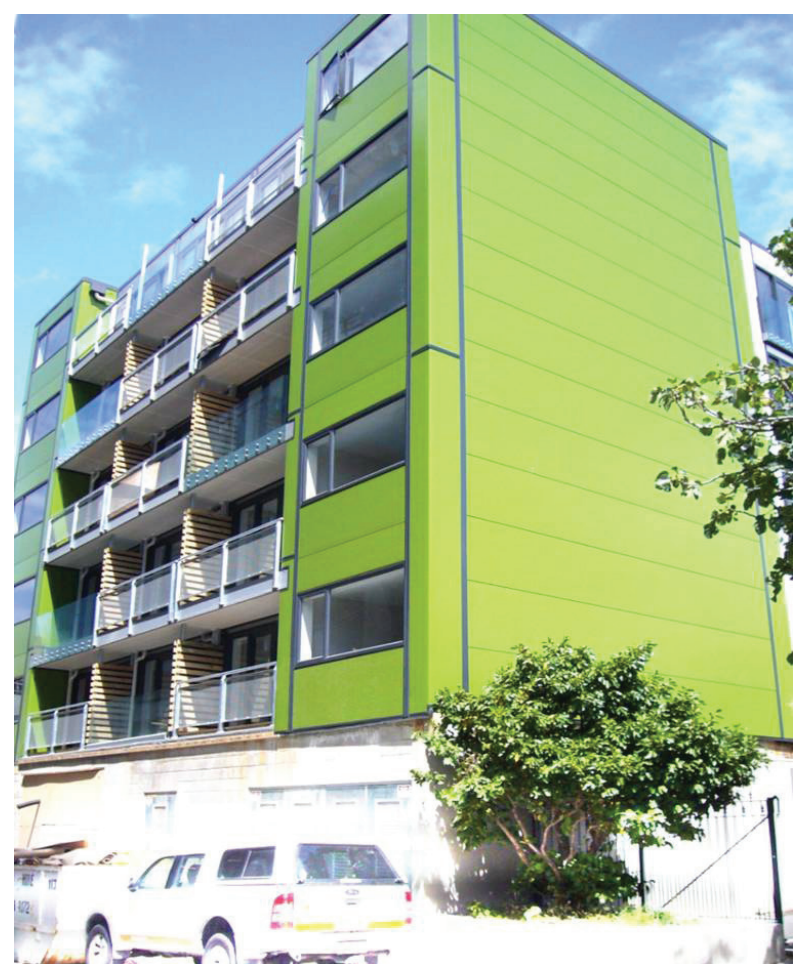



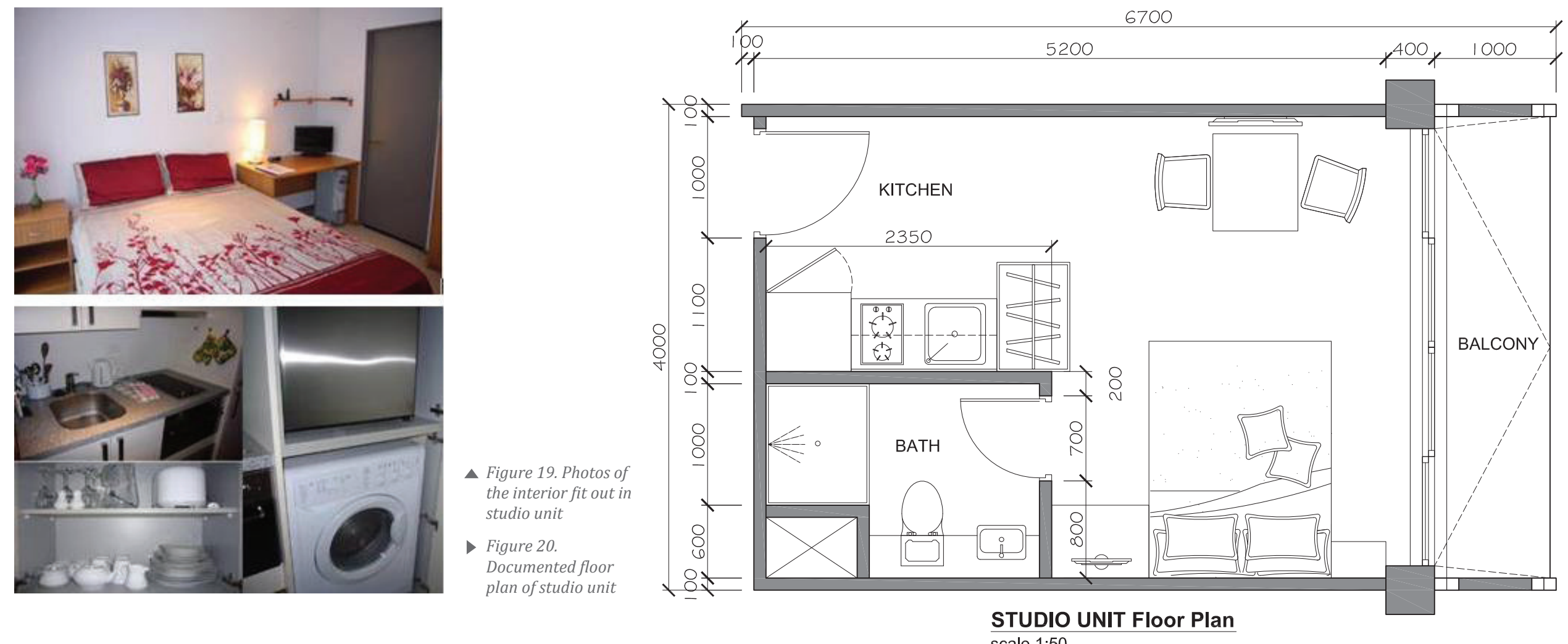

STUDIO UN

- Figure 19. Photos of the interior fit out in studio unit

- Figure 20

Documented floo plan of studio unit scale 1:50 


\subsubsection{SOHO Apartments}

- Floor area: $34 \mathrm{~m}^{2}$

- Balcony area: $2.8 \mathrm{~m}^{2}$

- Ceiling height: $2.8 \mathrm{~m}$

The fifteen-storey SOHO Apartments located on Taranaki Street contains one and two bedroom unit types with an exposed walkway and small balconies to each unit. It is popular for city dwellers as well as students due to its location. Similar to DUEL on Vivian, SOHO offers fully furnished rooms with private facilities. Onsite shared facilities including gym and indoor swimming pool are also offered. The floor plan shows one of the one bedroom apartments. Again, the kitchen is beside the narrow entrance and there are very limited storage options. Considering that this is targeted for couple occupants, the one metre wardrobe is way too small. Two sliding doors allow the bedroom which has no windows to be opened up. Even so, the amount of sun light that could be gained in the bedroom is questionable.

Figure 21. SOHO Apartments street view and bedroom with insufficient space

- Figure 22. Type E one bedroom apartment floor plan
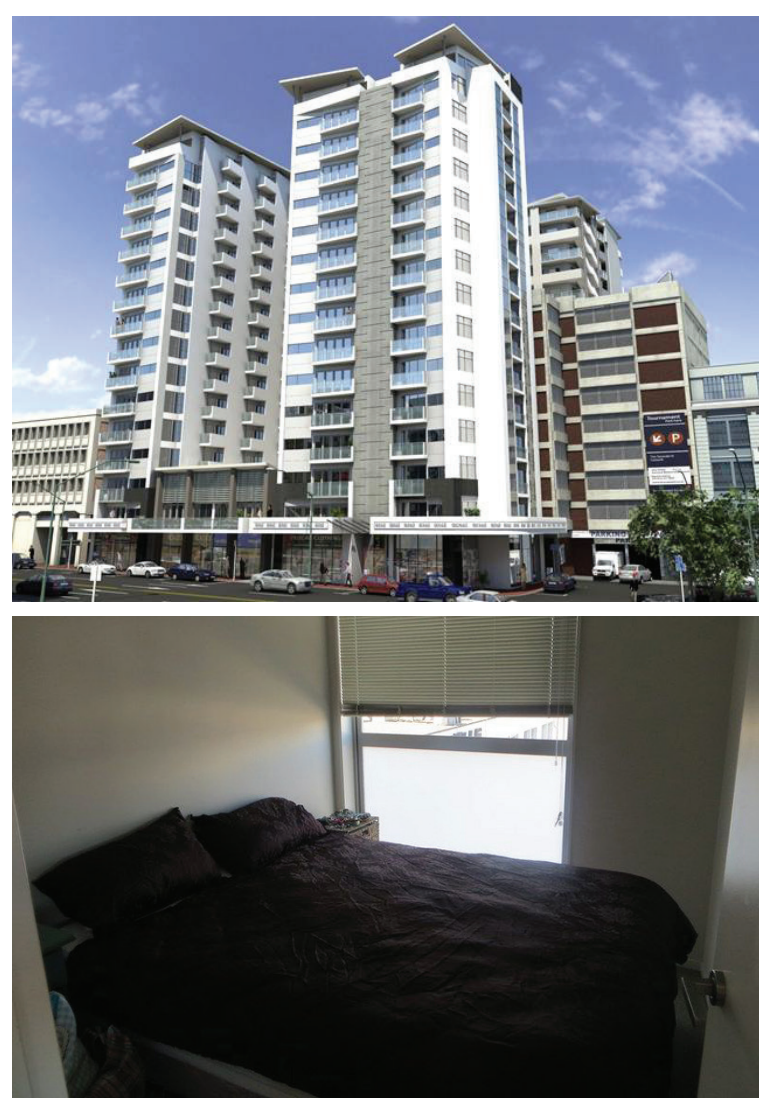


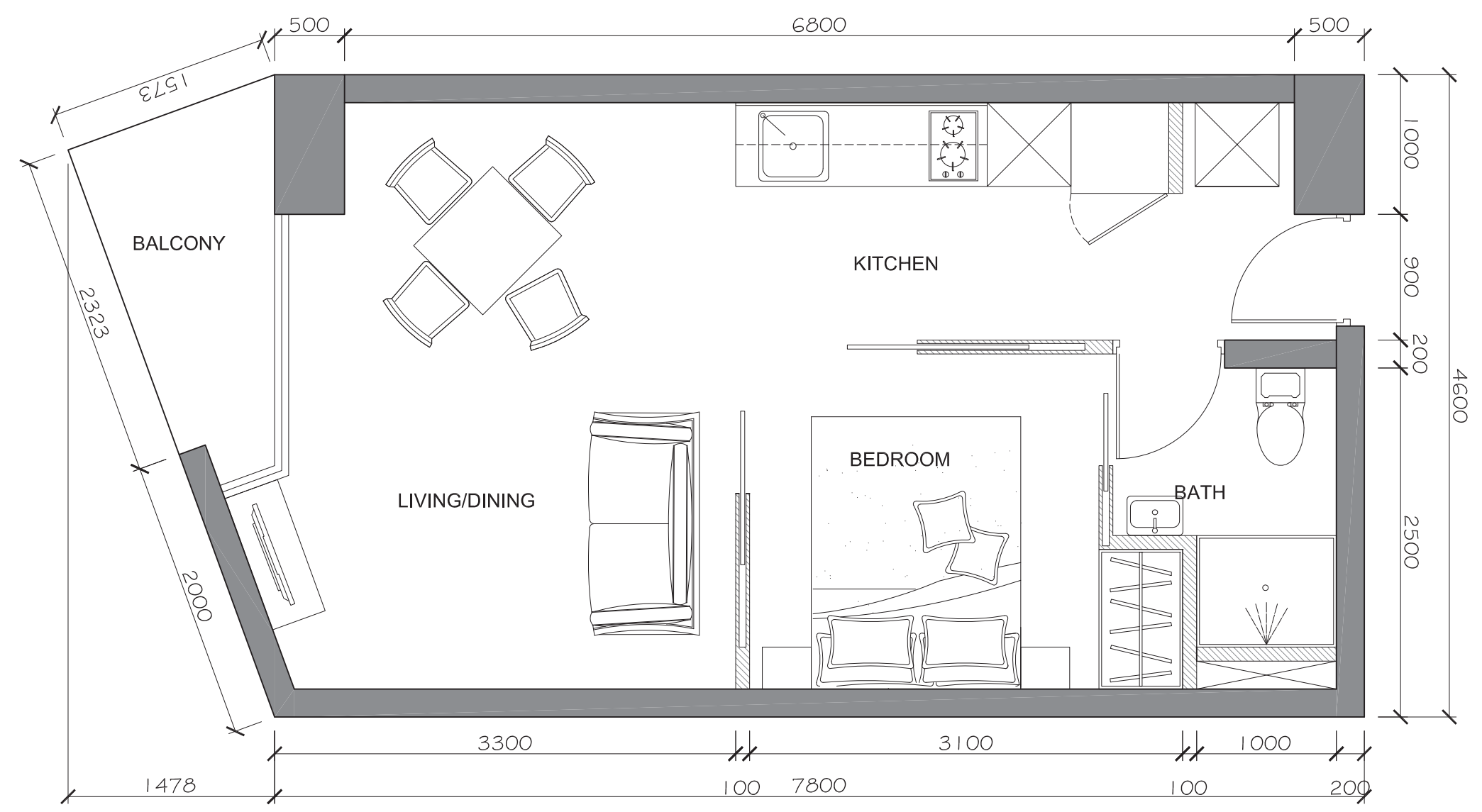

TYPE E Floor Plan

scale 1:50 


\subsubsection{TE PUNI Village}

- Floor area: $26.5 \mathrm{~m}^{2}$

- Ceiling height: $2.8 \mathrm{~m}$

Te-Puni Village is one of the university halls operated by Victoria University of Wellington. It is located at the southern end of Kelburn Campus on a steely sloping site. Because of this, the village has been divided into three blocks named the tower, the edge and the terrace with different heights and a sky bridge connecting them. As a high density living project, it provides accommodation for 389 students in a mix of single bed dormitory, one bedroom and two bedroom apartments. The floor plan shows one of the studio types in the terrace building that includes a private bathroom, but most rooms in the tower and edge blocks have no private facilities. They instead share toilets, showers and kitchens located on each floor. Higher living density may be achieved by sharing private facilities, but it may not form a comfortable quality living experience. One interesting aspect proposed in this scheme was the shared common corridor. Unlike conventional apartments which have narrow corridors leading to private units, the picture on the right shows that Te-Puni has intentionally created a wider corridor that allows students to gather and utilise as a shared platform.

- Figure 23. Te-Puni Village street view

- Figure 24. Enlarged corridor used as shared platform
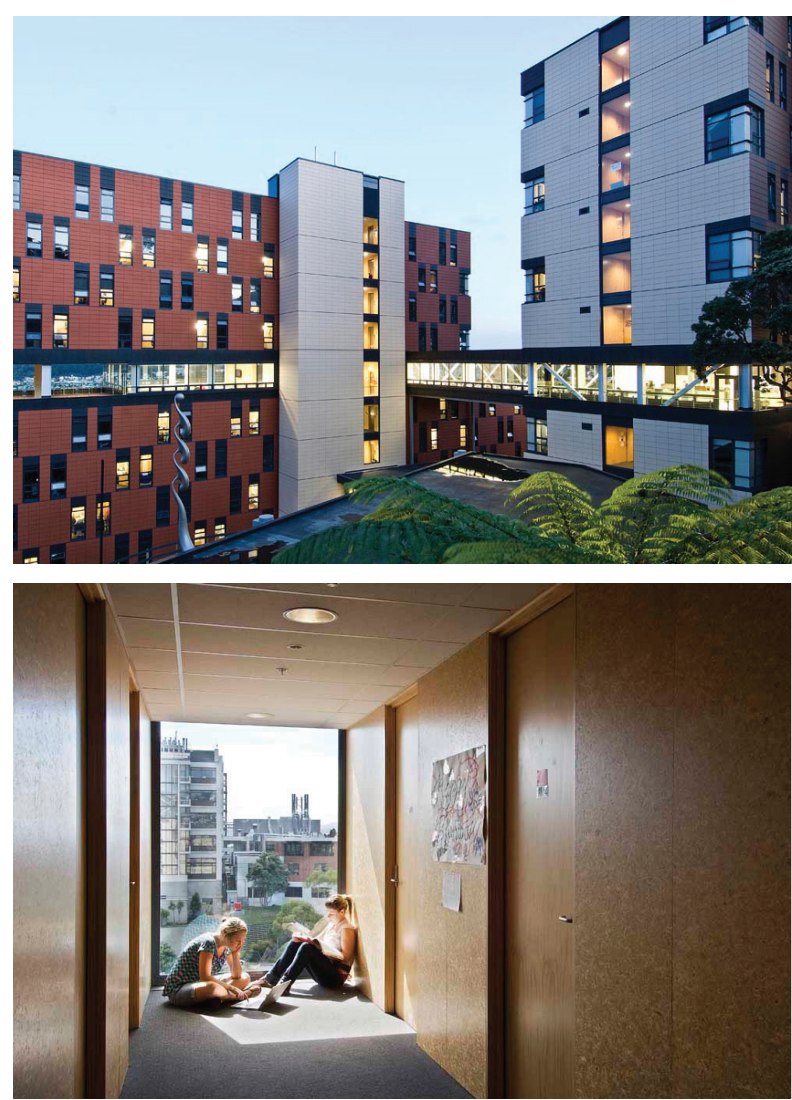

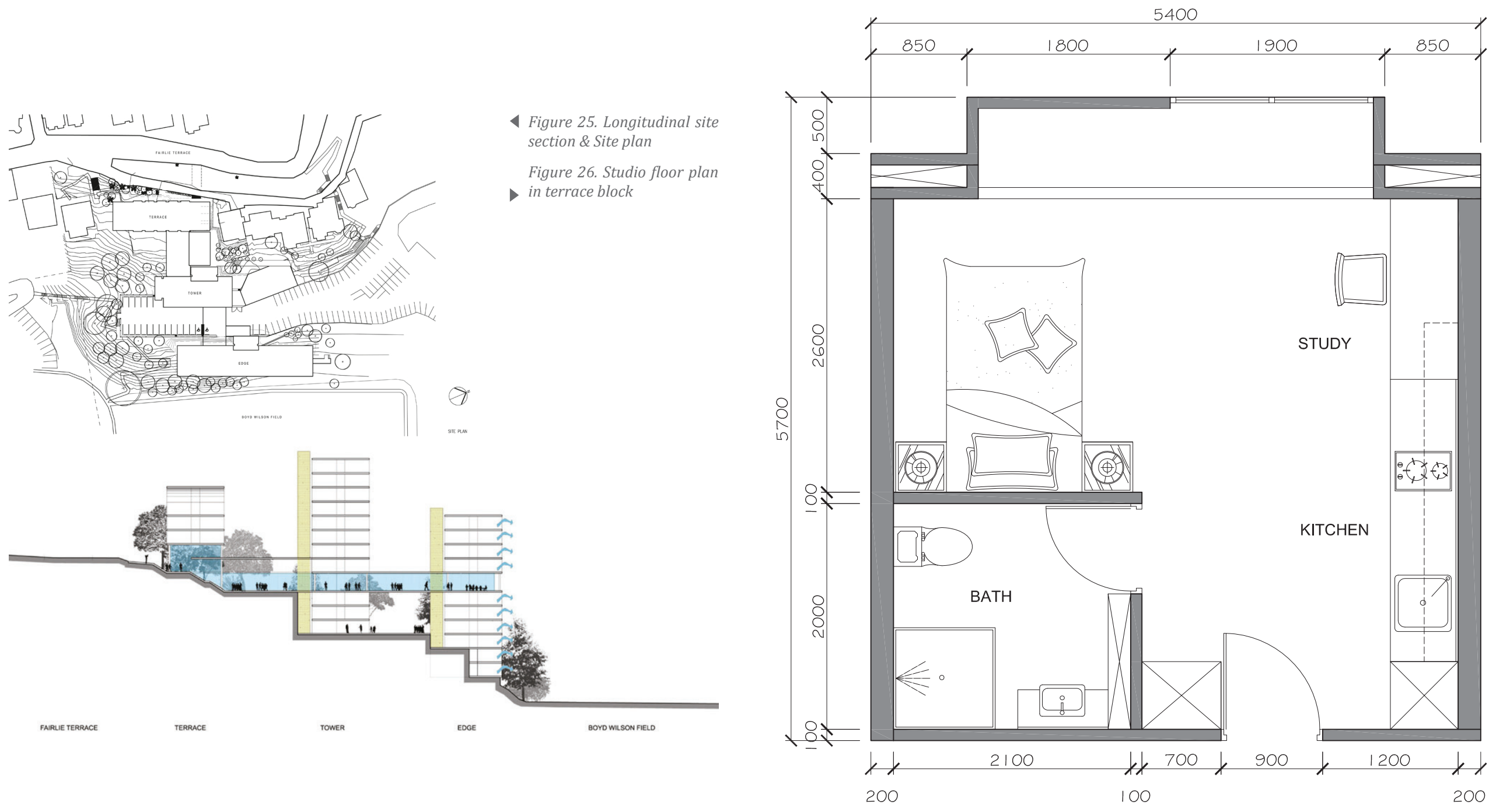

Studio Floor Plan scale 1:50 


\subsection{FINDINGS AND REFLECTIONS}

By comparing the documented cases of both local and international compact living projects, it is not hard to see that the studio and single bedroom apartments in Wellington are more generous in terms of internal space. However, the international cases show that a higher standard of use efficiency can be achieved in a smaller floor area. Most overseas compact housing solutions were carefully considered in relation to the space and functionality. In terms of offering necessary private facilities, it seems that a comfortable and functional habitable space can be created a floor area of 15 to $20 \mathrm{~m}^{2}$. Spaces less than $15 \mathrm{~m}^{2}$ usually compromise by sharing kitchen and/or bathroom facilities. Spaces larger than $20 \mathrm{~m}^{2}$ are considered too luxury for student targeted compact living, unless it is intended for two or more occupants. Rather than providing space with freedom, giving specific definition and suggestions on the use of spaces seem to be a better solution to prevent wasted or underutilised space. Reflecting on the international projects, efficiency was certainly one of the major factors that affects the quality and experience of living in small space. This idea of efficient use of space is one missing element in most local cases. Because of this, design phase one will investigate the possible ways of achieving internal efficiency in compact dwellings. 


\section{DESIGN PHASE I: HYPER EFFICIENCY}

A common goal of designing for compact housing is to make the space feel larger than it actually is. As Stwart (2004) suggests, "the homes I like the best are completely occupied, busy, and useful. I find smaller spaces - comfy spaces - better than great big houses that are hardly used." (p. 224). Making the most use of limited space is a crucial factor that determinates the quality of compact housing. In order to achieve such living quality, design iterations of compact individual units were tested in this chapter, with a focus on overcoming the constraints of restricted space through hyper efficiency.

\subsection{COMPACT SINGLE UNIT: MAXIMISING SPACE}

Instead of physically expanding spatial boundaries, design iterations in this part of the chapter set out to test possible ways to maximise a restricted space. "Using less space does not mean cramming amenities into uncomfortable quarters" (Richardson, 2011). The challenge here was to fill in the functions in less space yet with the sense of comfort. The first design investigation looked at how space can be transformed into different functional areas with the use of mobile furniture and walls. The second investigation capitalized on findings from the previous iterations and expanded the area of study to test the proposition of how the space can be split and defined according to different functions instead of confining all activities to one space. 
4.1.1 Space Transforming: Multi-Functional

Components

In this section the design tests the ideas of how to allow a limited space to serve multiple purposes. The idea of transforming was tested by introducing movable furniture and walls to reform the same space into a changed environment. Tengbom Architects came up with the smart student unit ${ }^{[1]}$ in Sweden (Figure 27) and suggested the actual time spent by students in their rooms is limited; hence the living space can be reduced to minimum. Many of the internal elements do double duty as furniture and structural components, which they believe it is the perfect answer to form meeting function in a limited footprint. The Egg House mentioned

1 inthralld. (2013). 100 square foot smart student unit in sweden. in the previous chapter as a compact unit case study, shows how a tiny space is able to expand and begins a conversation with the external surroundings despite the fact that it is limited in facilities. Another example of maximising space with co-existence of a bed and storage cabinet was shown in the artist studio done by Rannan Stern. Both these case studies demonstrated ways of fully utilising limited space with the efficient use of furniture. By applying transformative techniques such as sliding and flipping, the design iteration demonstrated how a small space can be used in various ways to suit the users' needs at different times of a day.

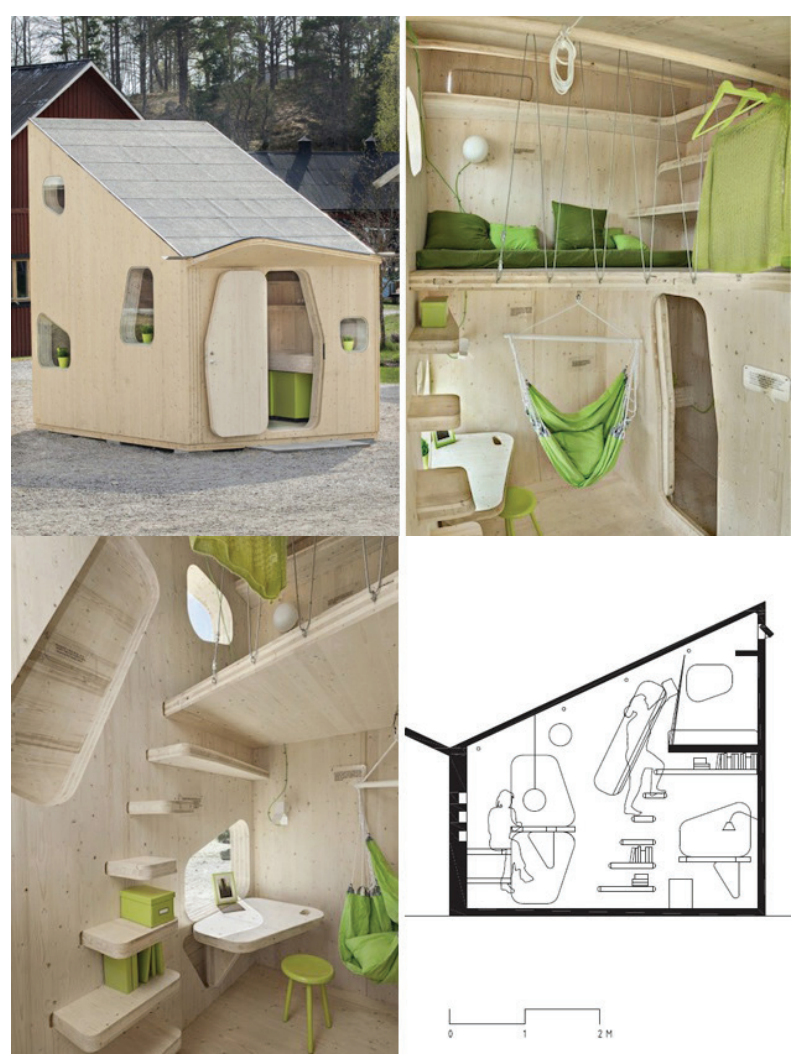

A Figure 27.100 square foot smart student unit 
The experiment utilising sliding and flipping furniture in this first design iteration demonstrates that the elevated bed is reachable via a set of drawers which slide out to be a staircase. The bottom step remains fixed on the floor and becomes a couch with a fold up backrest when the rest of the drawers are slid back under the bed. In terms of operable walls, an expansion of the internal space can be achieved by flipping down the bottom panel of the kitchen wall, so that occasional events such as a group of guests visiting can be held in a larger indoor and outdoor kitchen and living space.
On one hand the transformative space increases spatial efficiency, on the other it constrains the users' movement, which resulted in a negative user's experience. Questions such as how to increase the effectiveness of user exploration through transformative space has led to the next design iteration.

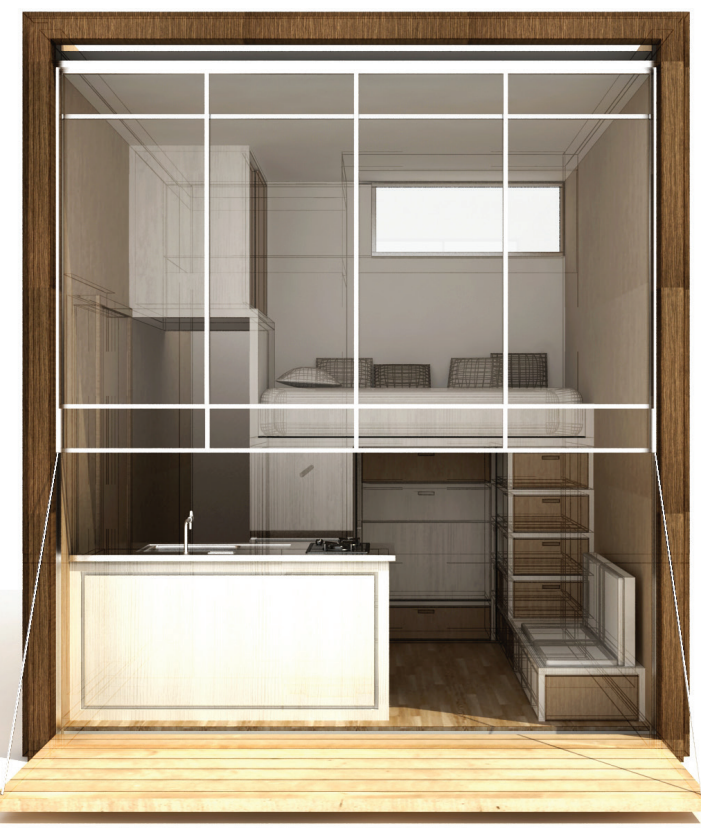

A Figure 28. Experiment using sliding and flipping furniture 

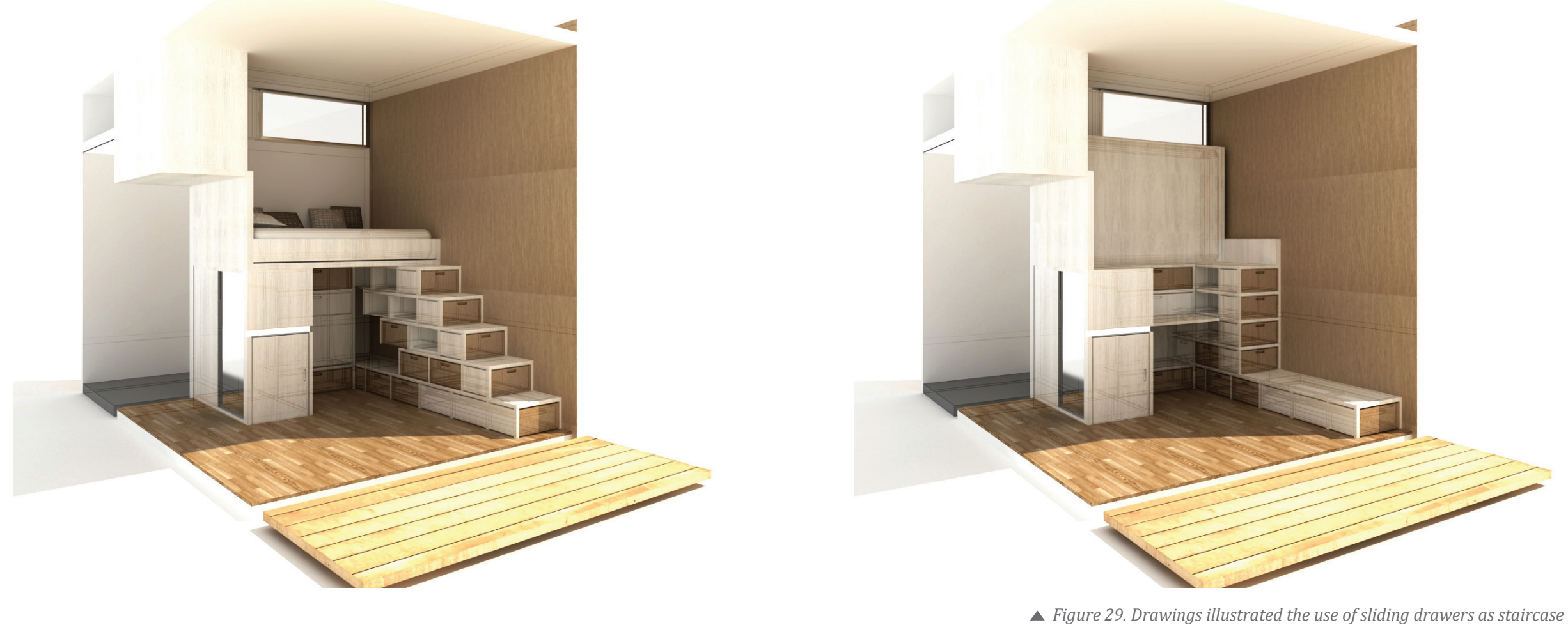
As Özsoy and GÖkmen (2005) suggest, "space use and furniture arrangements, in conjunction with the dwelling layout, are considered as two interrelated indicators of housing quality" ( $p$. 19). The second iteration intents to focus on the internal layout aspect which is looking at how to achieve a degree of privacy by separating restricted space and generate functional and spatial volumes for each individual function. The Cube Project documented in the previous chapter, successfully showed a way of living in compact space, by dividing the internal space into three overlapping floor levels. Giving some definitions to each functional area by splitting floor levels increases the range of user experience by allowing occupants to explore and settle in different designed spaces and environments as suits their daily routine and mood. The Cube suggests keeping each floor level at two metres height in order to minimise the wasted space.

Influenced from the Cube Project, this design iteration proposed a $10 \mathrm{~m}^{2}$ compact single unit with full facilities and overlapping spatial character. Entering from a $6 \mathrm{~m}^{2}$ ground level, a sliding table on the left hand side allows the space can be transformed between two to four seated living and dining area. A decent amount of storage cabinet is located on the right, with a space saving staircase that leads to the second level - a $5.7 \mathrm{~m}^{2}$ kitchen and bathroom. The bathroom is designed as a separated toilet and shower so to allow efficient occupation by a couple. Up the steps onto the top level is a $4.6 \mathrm{~m}^{2}$ bedroom with a double bed and wardrobe. 


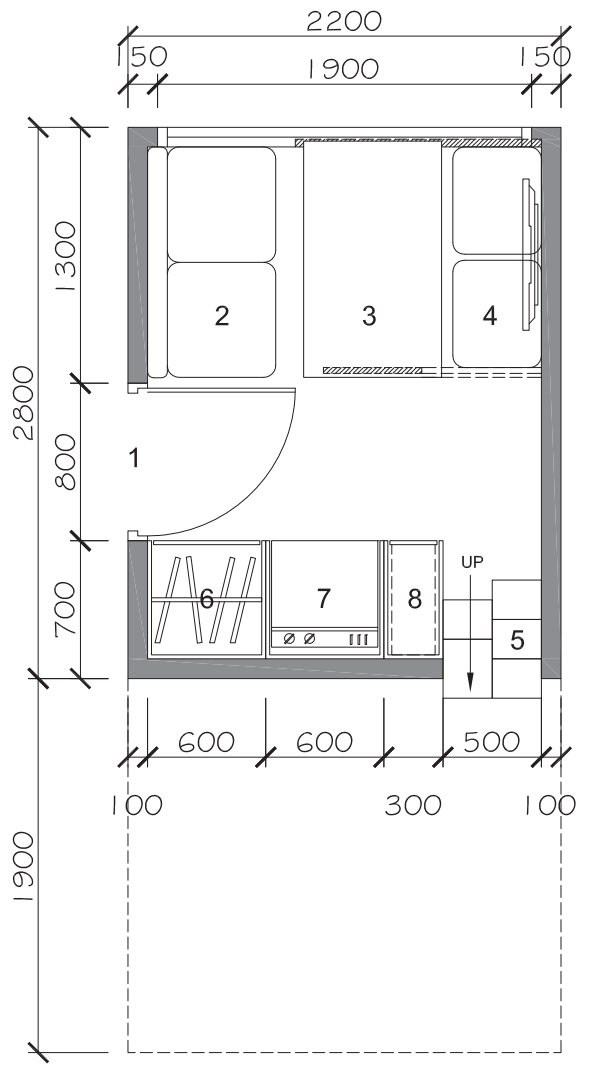

LEVEL 0 FLOOR PLAN

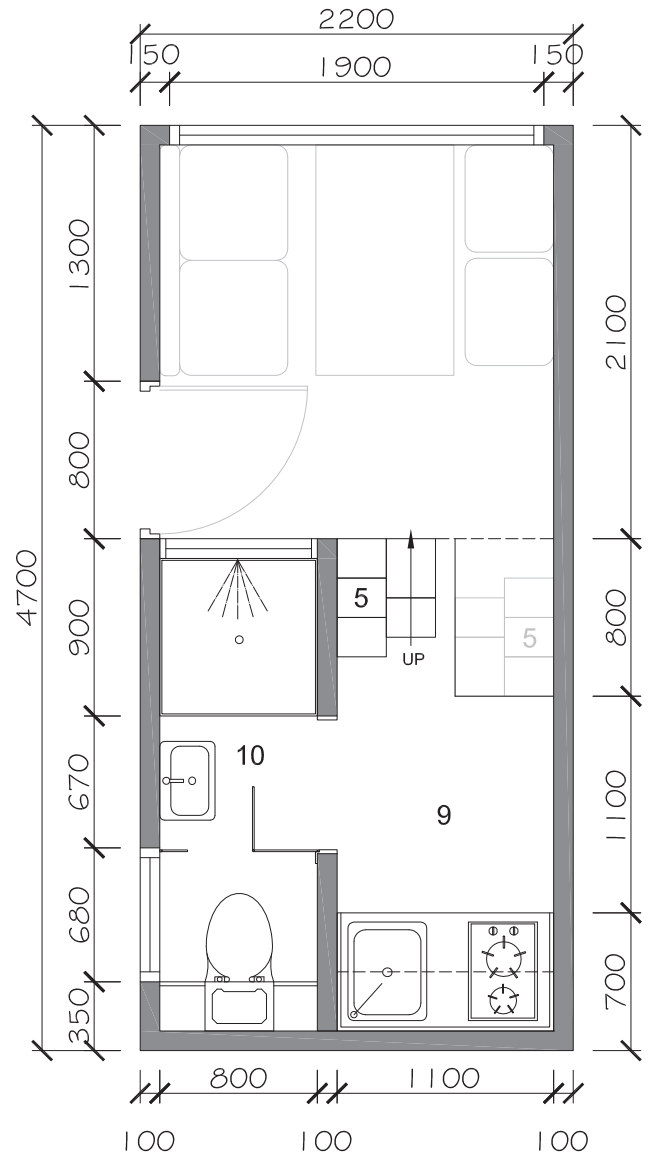

LEVEL 1 FLOOR PLAN

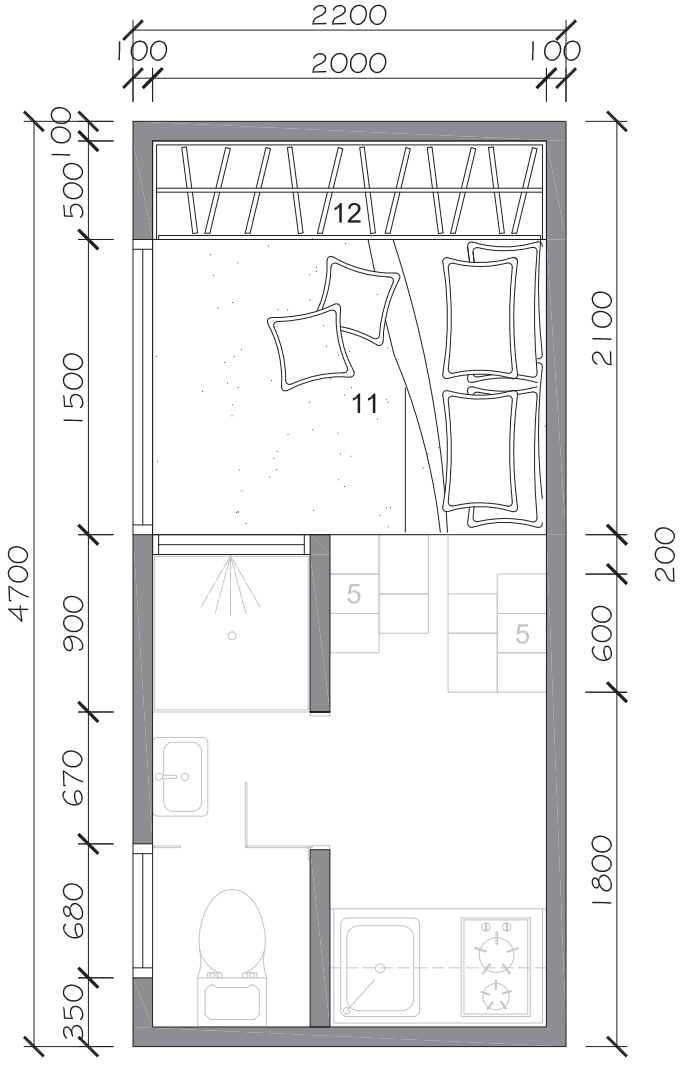

\begin{tabular}{lr}
\multicolumn{2}{l}{ LEVEL 2 FLOOR PLAN } \\
\hline BEDROOM
\end{tabular} 


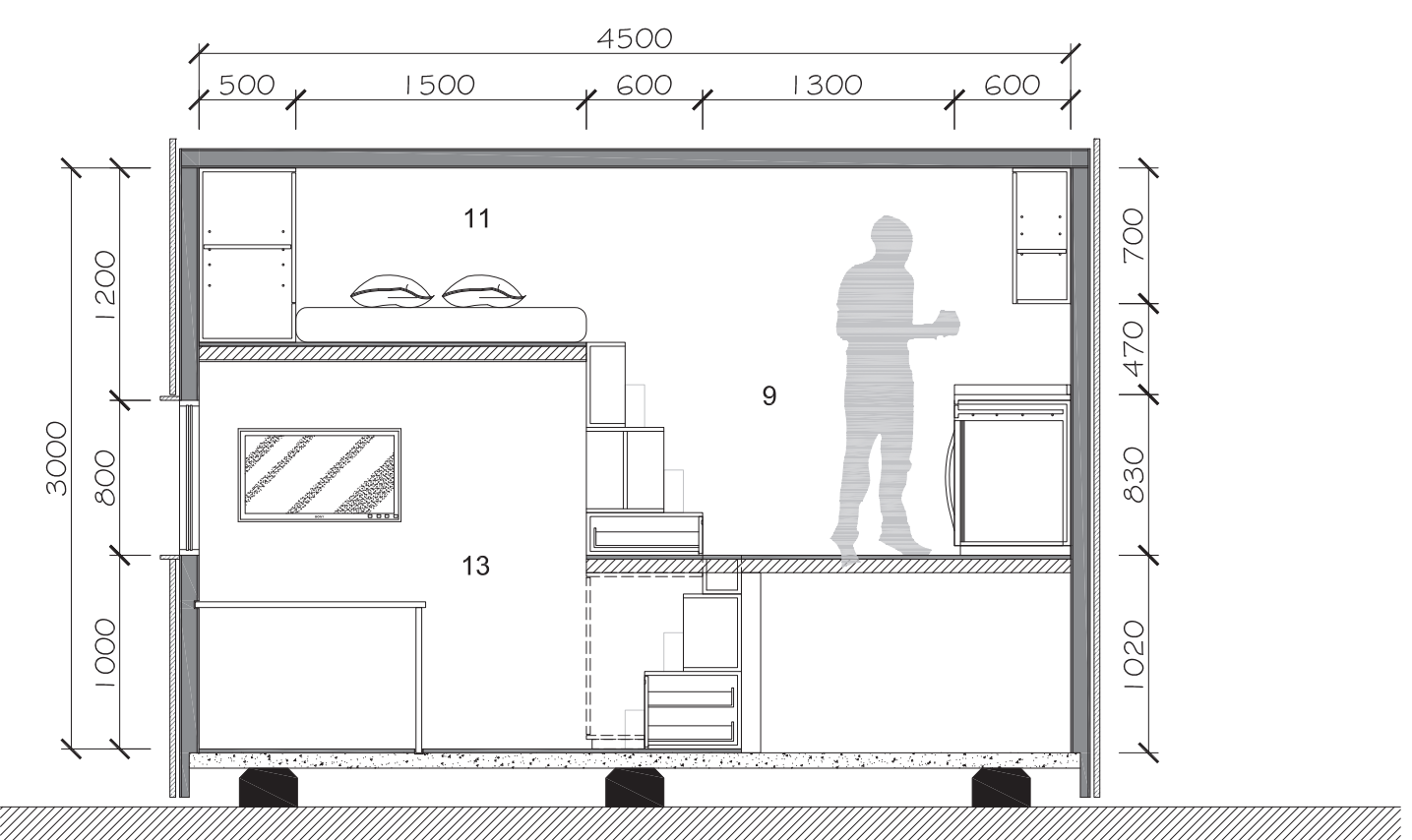

LONGITUDINAL SECTION

\ Figure 30. Floor plans of $10 \mathrm{~m}^{2}$ compact single unit

- Figure 31. Sections of $10 \mathrm{~m}^{2}$ compact single unit

1. MAIN ENTRANCE

2. CUSTOM-MADE TWO SEATED COUCH WITH

BOTTOM STORAGE

SLIDING TABLE

4. CUSTOM-MADE SEATING CUBES

SCALE 1:50

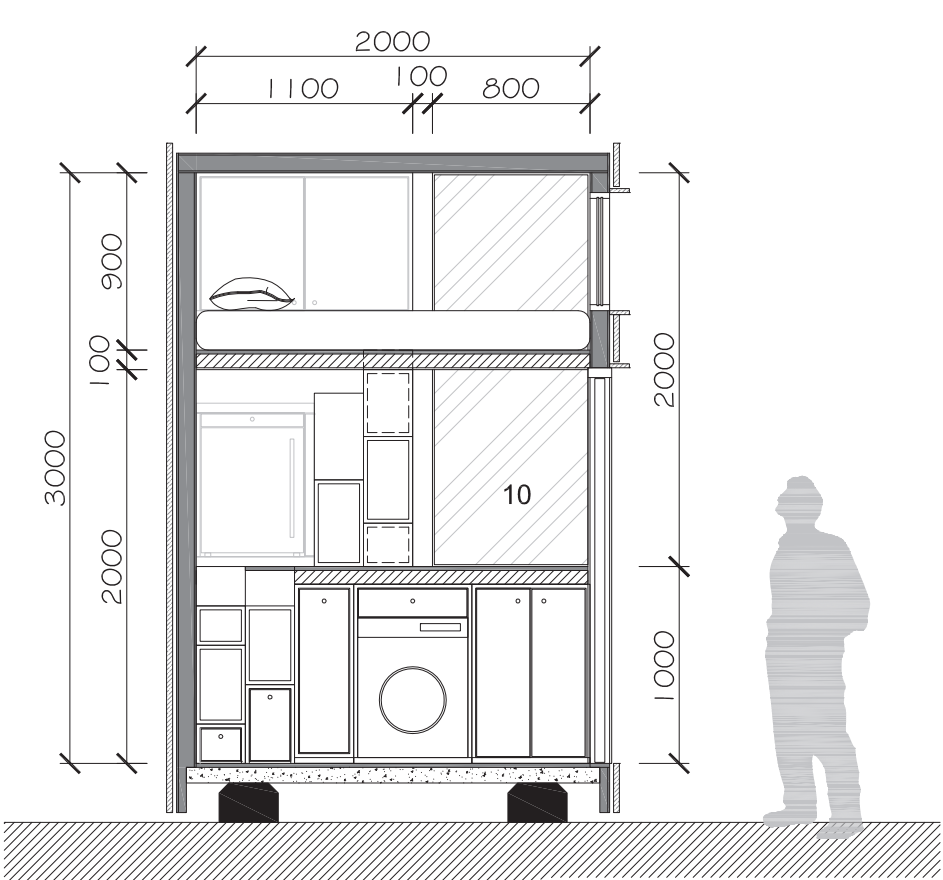

TRANSVERSE SECTION

SCAL150

10. TOILET \& SHOWER

11. QUEEN SIZE BED

2. WARDROBE / STORAGE CABINET

13. LIVING / DINING AREA 

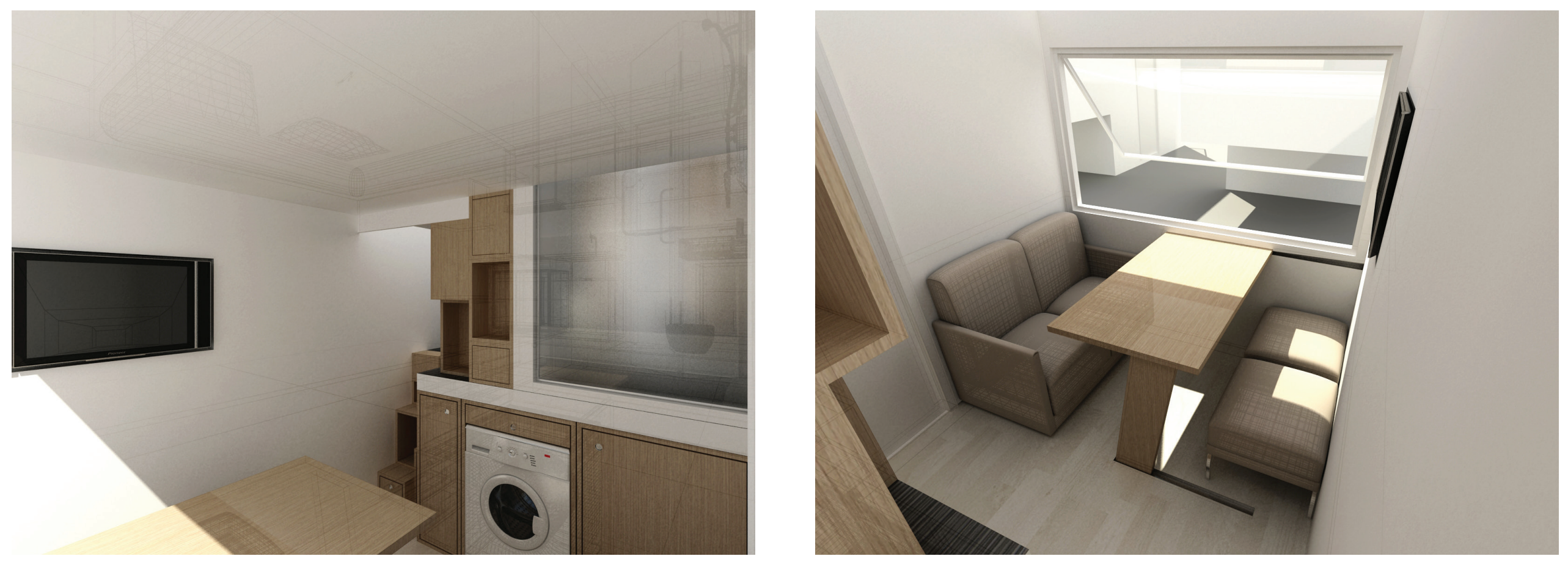

\ Figure 32. View from living area to staircase

- Figure 33. View from staircase to living area 

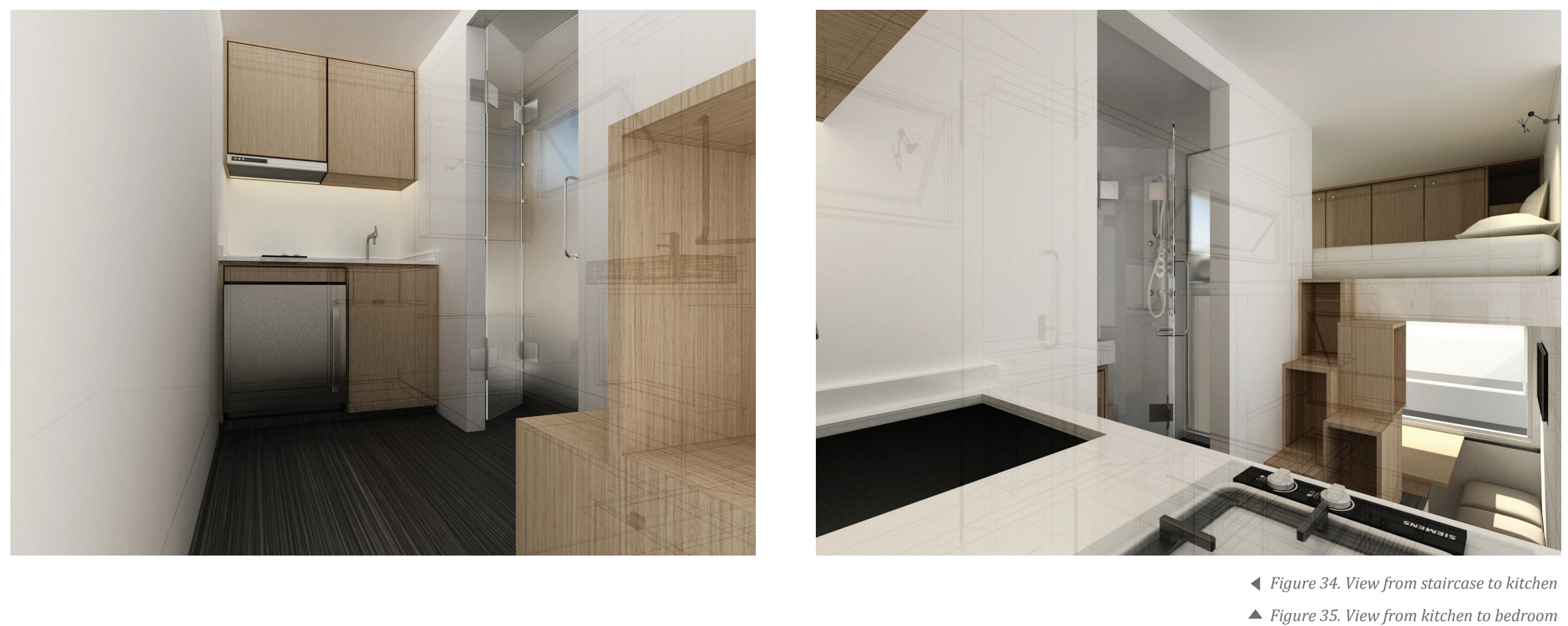

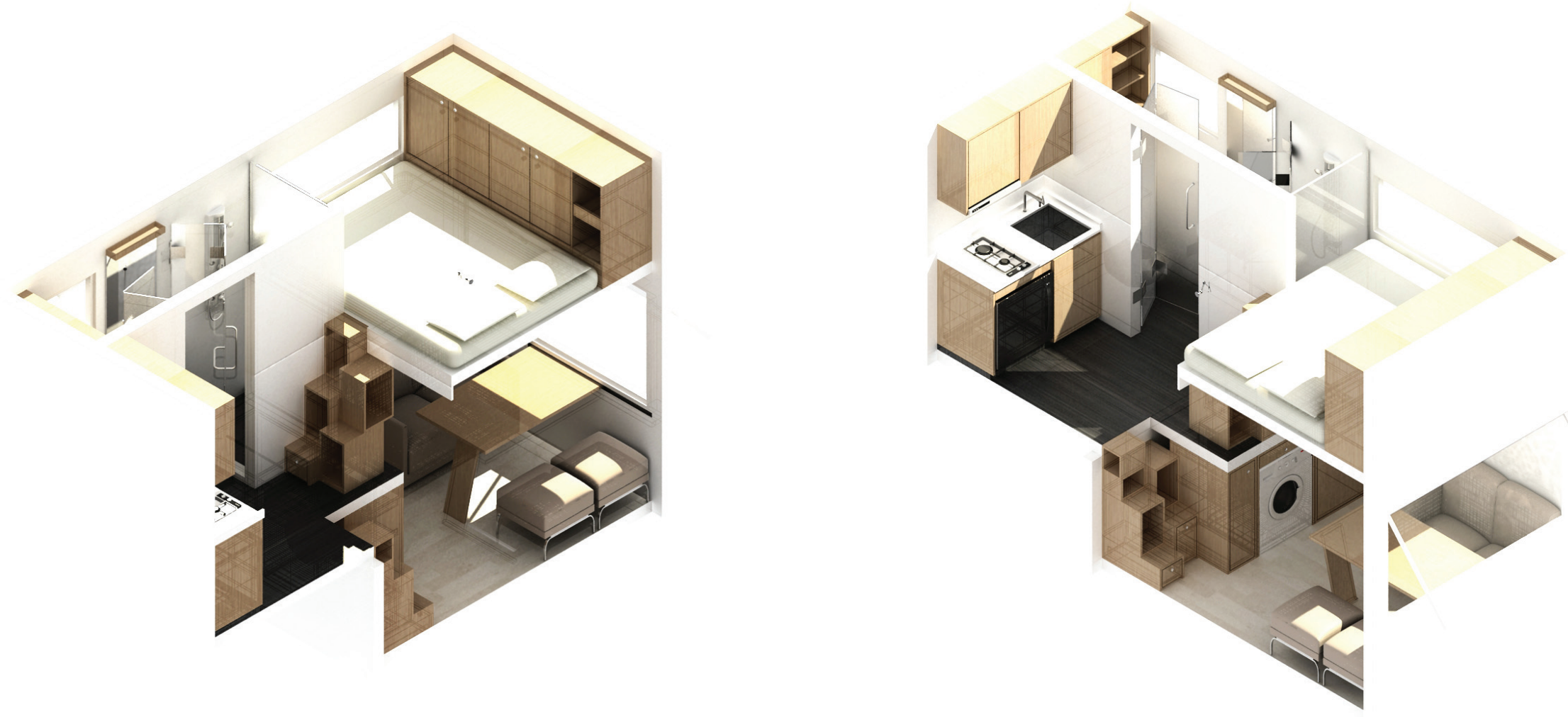

- Figure 36. Axonometric of $10 \mathrm{~m}^{2}$ compact single unit 
Compared to the previous design iteration, this proposal not only offers the option of exploration through the unit, but also increases the utilised floor area in a confined space. By breaking and separating the functional areas into different defined territories, the result shows that the possibilities of how a tiny studio can be occupied and felt as an expanded one bedroom unit. One issue that can be certainly improved in the next investigation is the restricted $2 \mathrm{~m}$ ceiling height on each level, which may not be consider as quality space for taller people. The design iterations to this point have been focused on internal efficiency in single units, however, in order to address the varying needs of student housing, there is an obvious need to have different unit types. Hence, the next step in the investigation will focus on the design of two bedroom units. 
Following the investigation of compact single units, the research expanded slightly to explore ideas such as a physical expansion of the space. This was attempted with a final goal of creating a space that would be suitable for two individuals in this part of the investigation. Unlike single units, a two or more bedroom
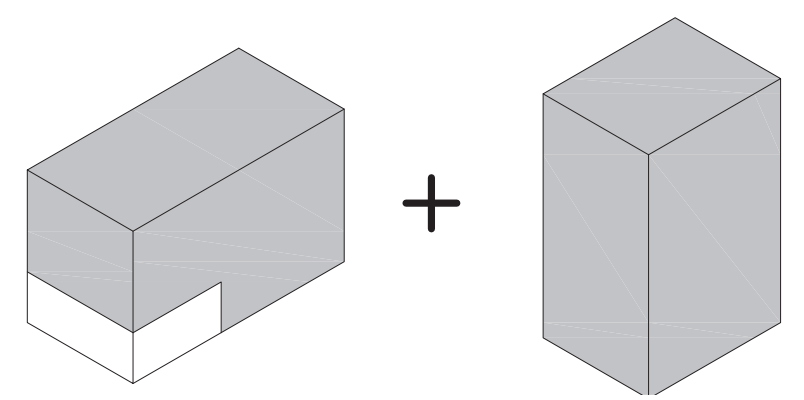
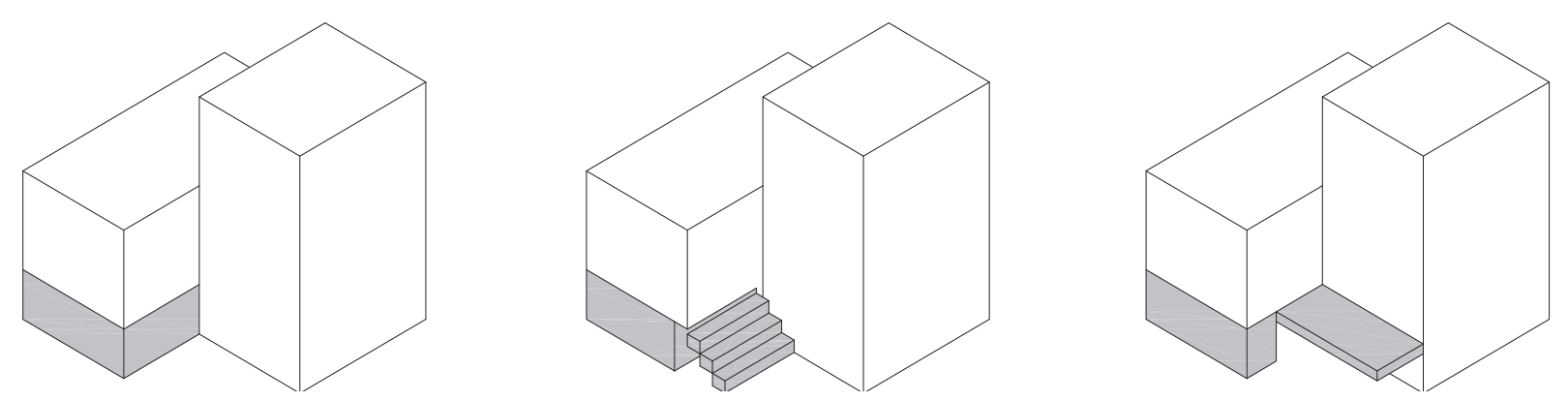

unit requires space for private uses, as well as common area that allows individuals to share. The hierarchy of private and public transition in a two bedroom unit is more complicated than for a single unit for one person, or a couple occupants. This design iteration considered how hyper efficiency can be applied to a space which needs common and private separations.

\subsubsection{Sharing in Private}

In order to create a visual diversity but avoid over expanding the space, the two bedroom unit module was designed by placing an identical vertical unit beside the original single unit. This 
design offers two double-size bedrooms with shared kitchen, toilet, study, and living. The two bedrooms are placed separately in two blocks in order to provide the necessary privacy. Unlike the single bedroom unit, extra ceiling height on the top level is given to improve the spatial quality in the bedrooms. The limited space is now extended so a study/dining area is created to suit the needs of students who prefer working at home. The kitchen is now shifted to the ground floor to keep a distance to the bedrooms. Bathroom remains at a middle level to provide convenience to both the common area and the bedrooms.

$\boldsymbol{\nabla}$ Figure 37. Design exploration on combining two single units together

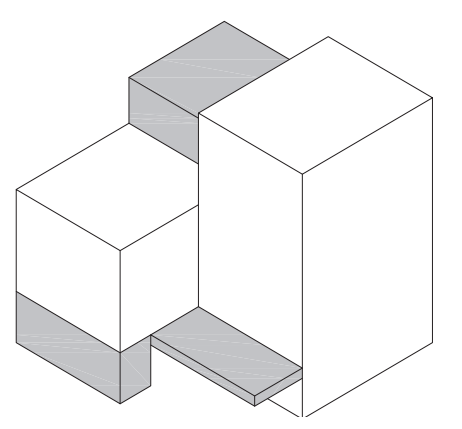

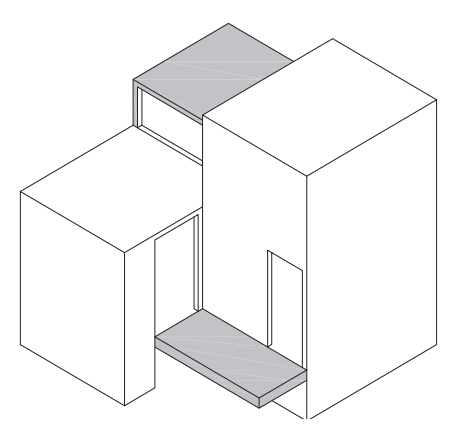

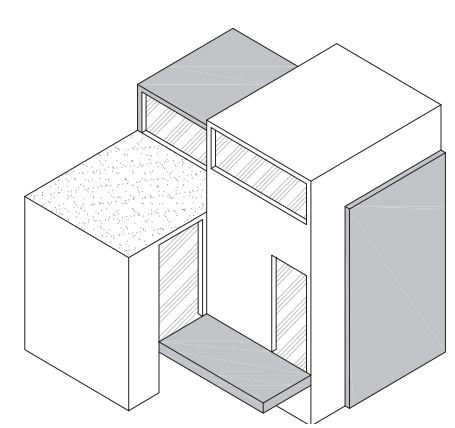



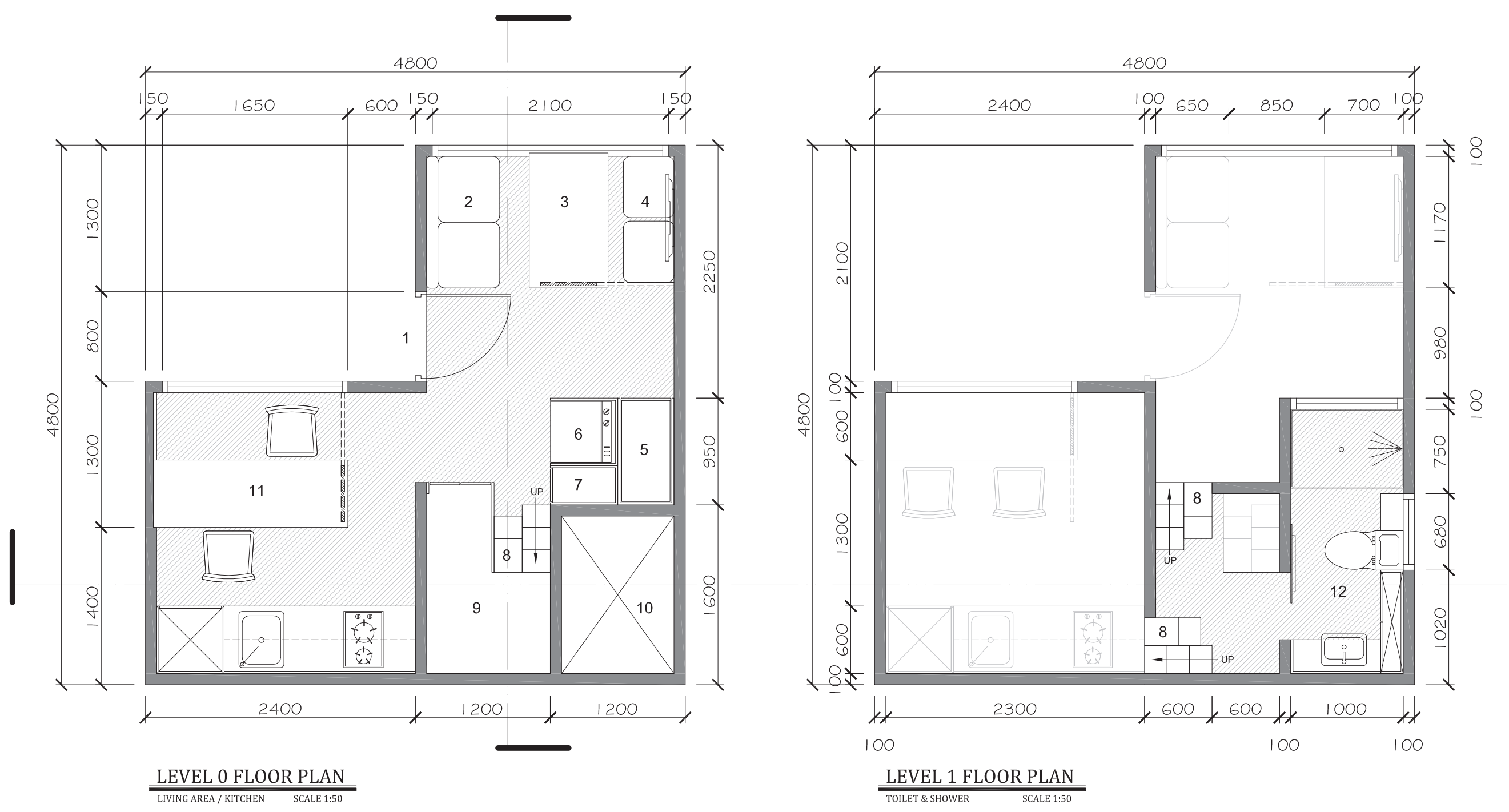


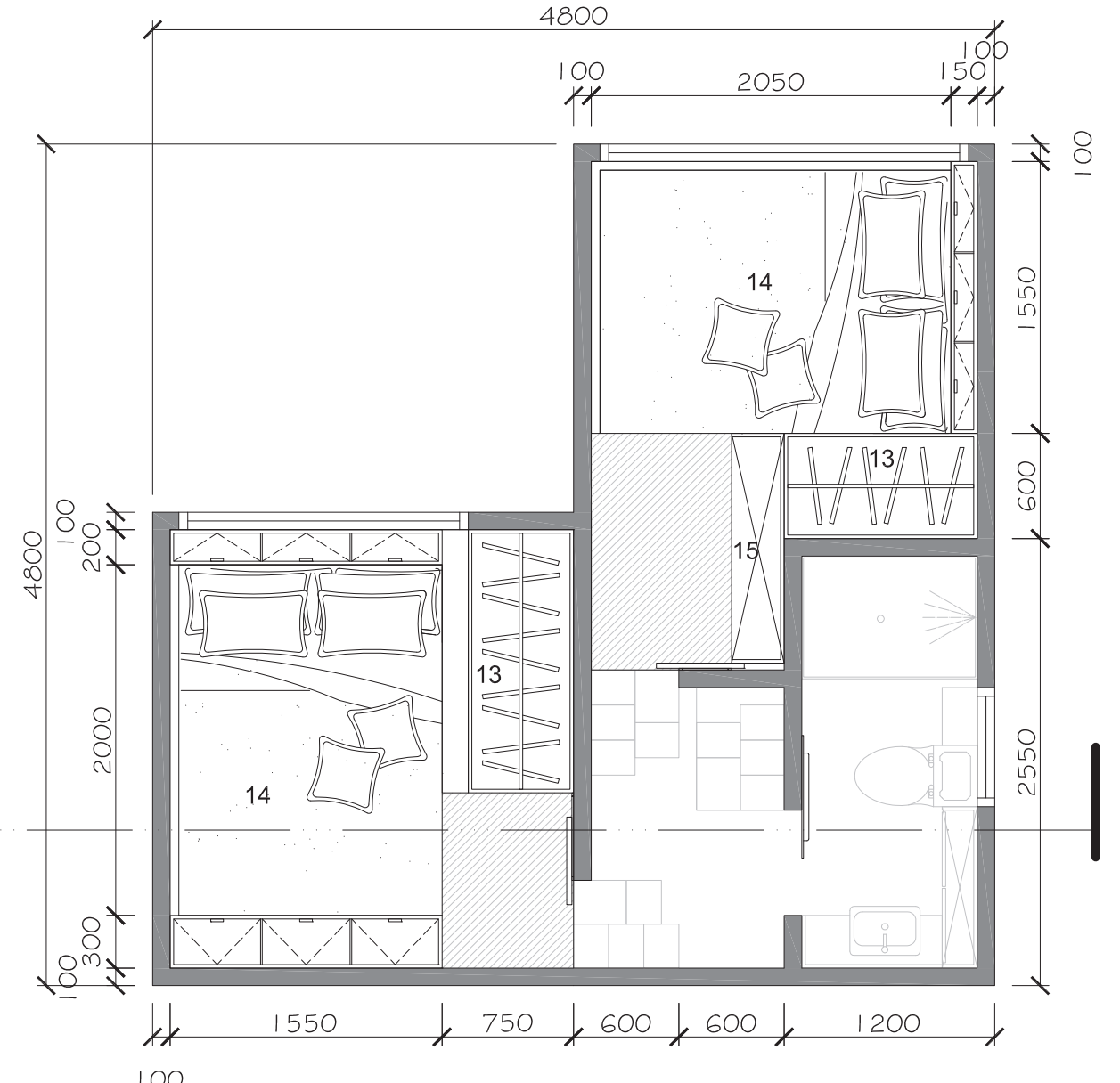

4 Figure 38. Floor plans of two bedroom unit

1. MAIN ENTRANCE

CUSTOM-MADE TWO SEATED COUCH

C/W BOTTOM STORAGE

SLIDING TABLE

CUSTOM-MADE SEATING CUBES

STORAGE DRAWER CABINET

BUILT-IN WASHING MACHINE

CASEMENT DOOR CABINET

SPACE SAVING STAIRCASE

1 METER HEIGHT STORE ROOM

10. SERVICES SPACE

11. SLIDING WORKING TABLE

12. BATHROOM

13. SLIDING DOOR WARDROBE

14. CUSTOM-MADE STORAGE QUEEN SIDE BED

15. OPEN SHELF STORAGE CABINET

LEVEL 2 FLOOR PLAN 


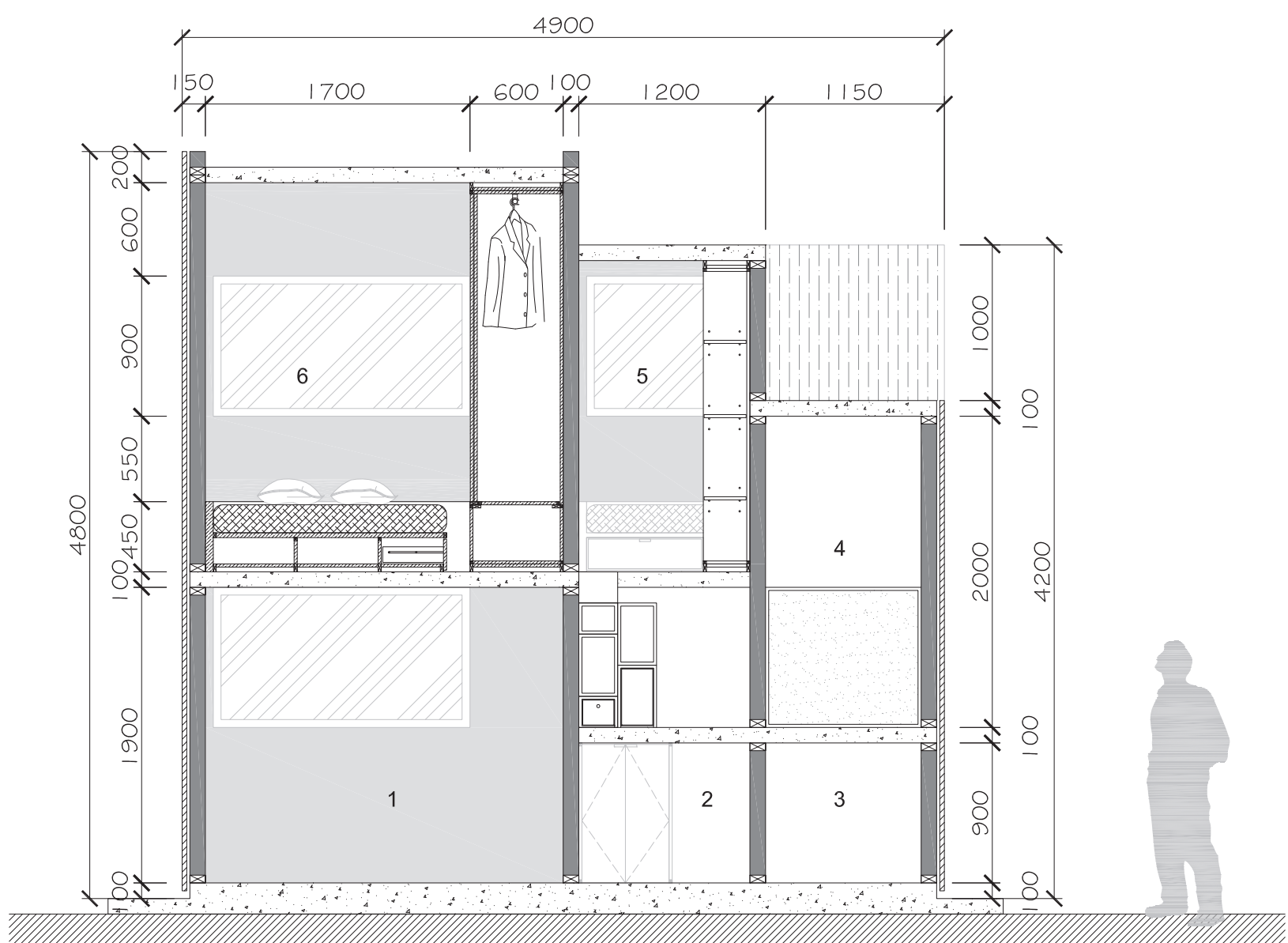

TRANSVERSE SECTION 


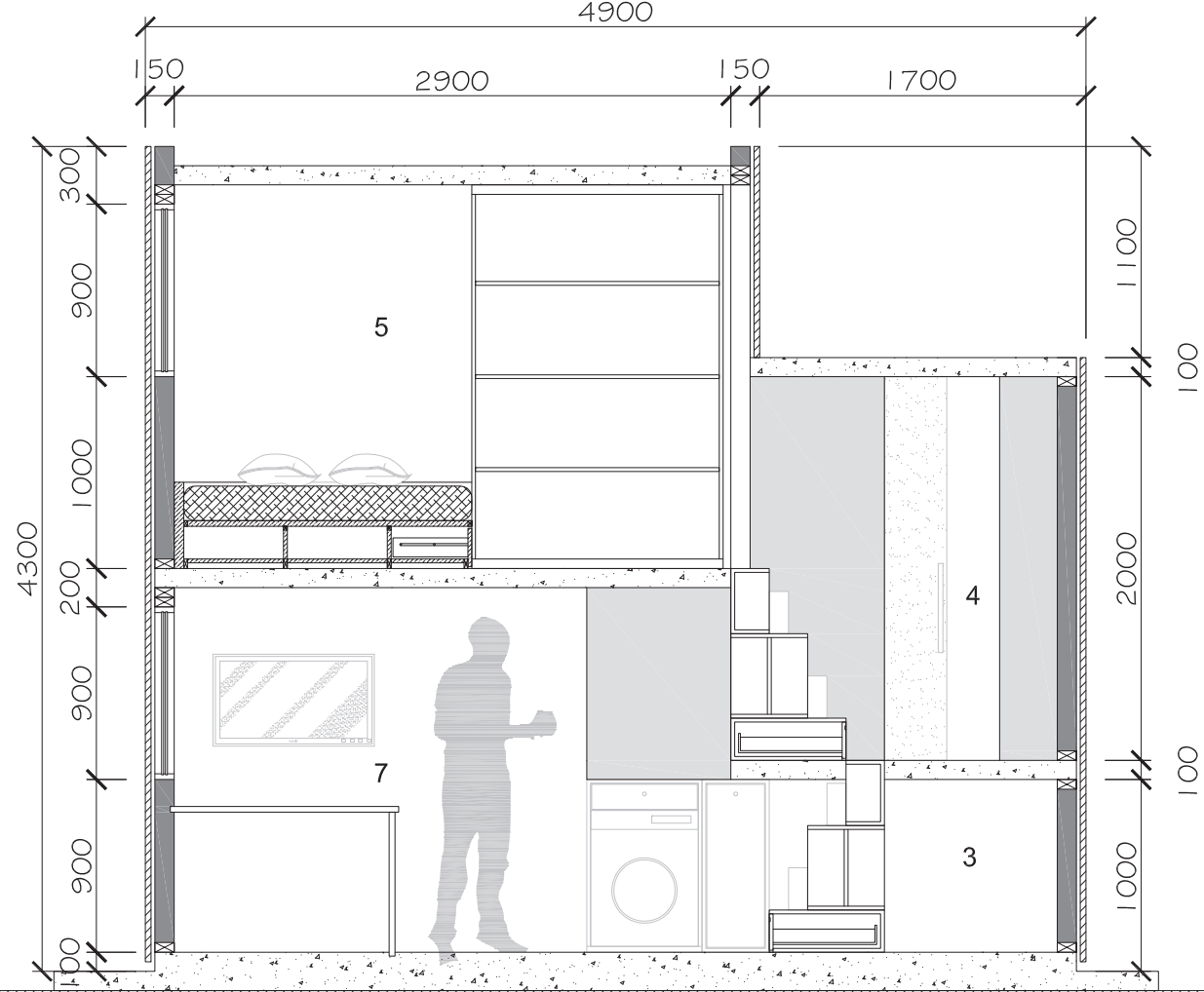

\ Figure 39. Sections of two bedroom unit
1. KITCHEN / DINING AREA
2. 1 METER HEIGHT STORE ROOM
3. SERVICES SPACE
4. TOILET \& SHOWER
5. BEDROOM A
6. BEDROOM B
7. LIVING AREA

\section{LONGITUDINAL SECTION}

$$
\text { SCALE 1:50 }
$$


Through the investigation of shared facilities with private needs, this proposal delivers a way of creating share space to link and attach two individual units together. The quality of such space can be evaluated as achieving two totally separated bedrooms which are accessible from individual stairs, as well as providing options for the residents to occupy the common space in a semi-private way.

\subsection{FINDINGS AND REFLECTIONS}

As a result of Design Phase I, the investigations of both single and two bedroom units concluded that quality of life in compact individual units can be improved with hyper efficiency. This can be achieved through transformative space with multi-functional components, as well as by creating internal defined spaces that give spatial occupation options to the occupants and by utilising the transition between shared and private spaces. The next design phase will focus on the factors that would improve the quality of life in larger scale complex with a group of units. The intention of such shift was to investigate how the idea of hyper efficiency can be applied into a medium or higher density housing environment with diverse degrees of circulation and connection between multiple units. 


\section{DESIGN PHASE II: SHARED SPACE}

In the second stage of this design thesis, the focus of the investigation shifted from efficient interior layout of individual units into creating the circulation and common space in between units and testing how the common space would be shared. The balance of public and share space was tested through design iterations of medium or higher density apartments. Such investigation brought up the awareness of positive sharing and the importance of creating transitional territories in between private and public space.

5.1 COMPACT SINGLE UNIT COMPLEX: SPACE IN BETWEEN

The intention of the first design attempt on this stage was to look at the spatial relationship between multiple individual units. A specific site was chosen to test how the circulation and common space in between would affect the quality of medium density community living environments. 


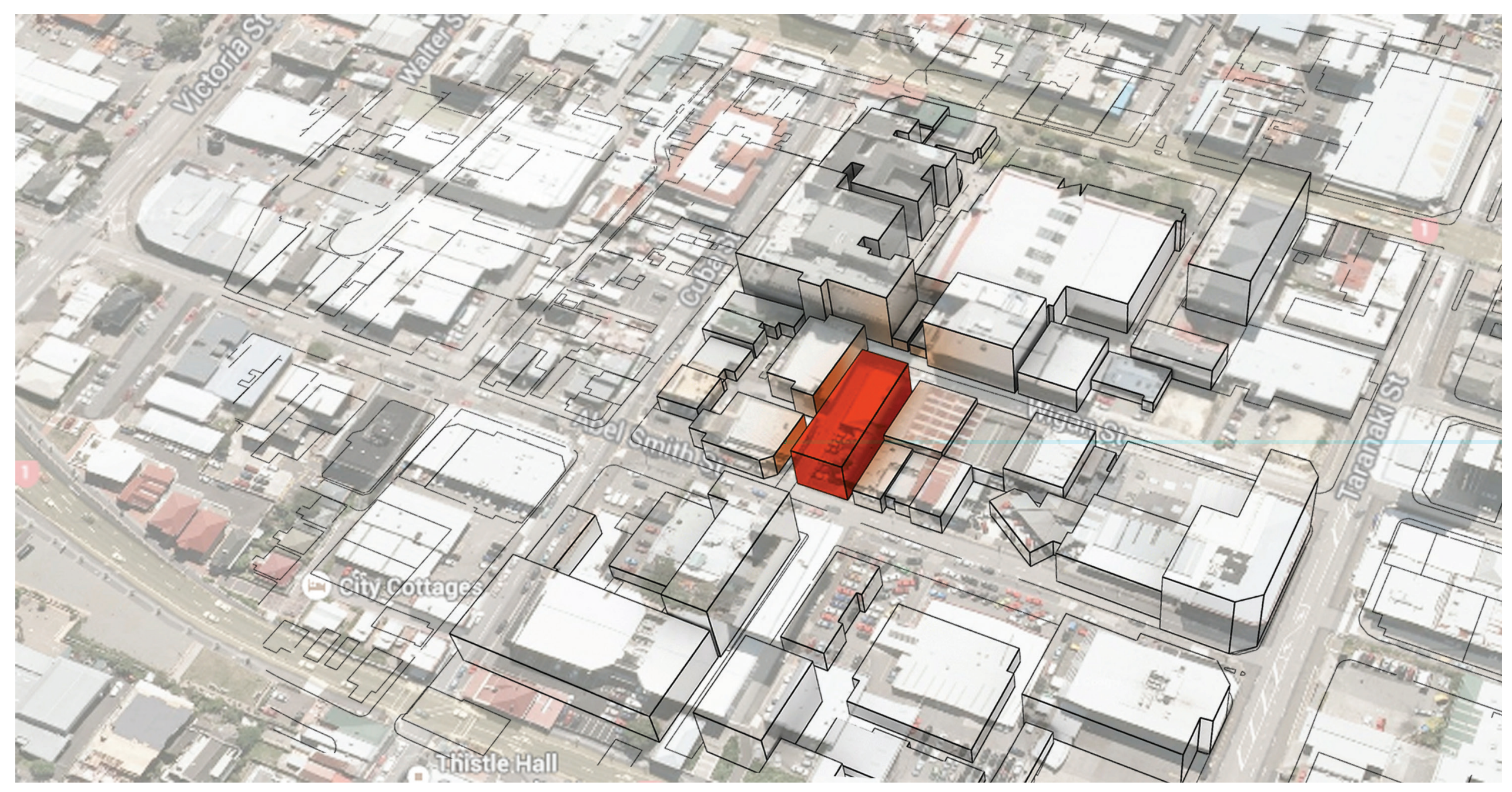




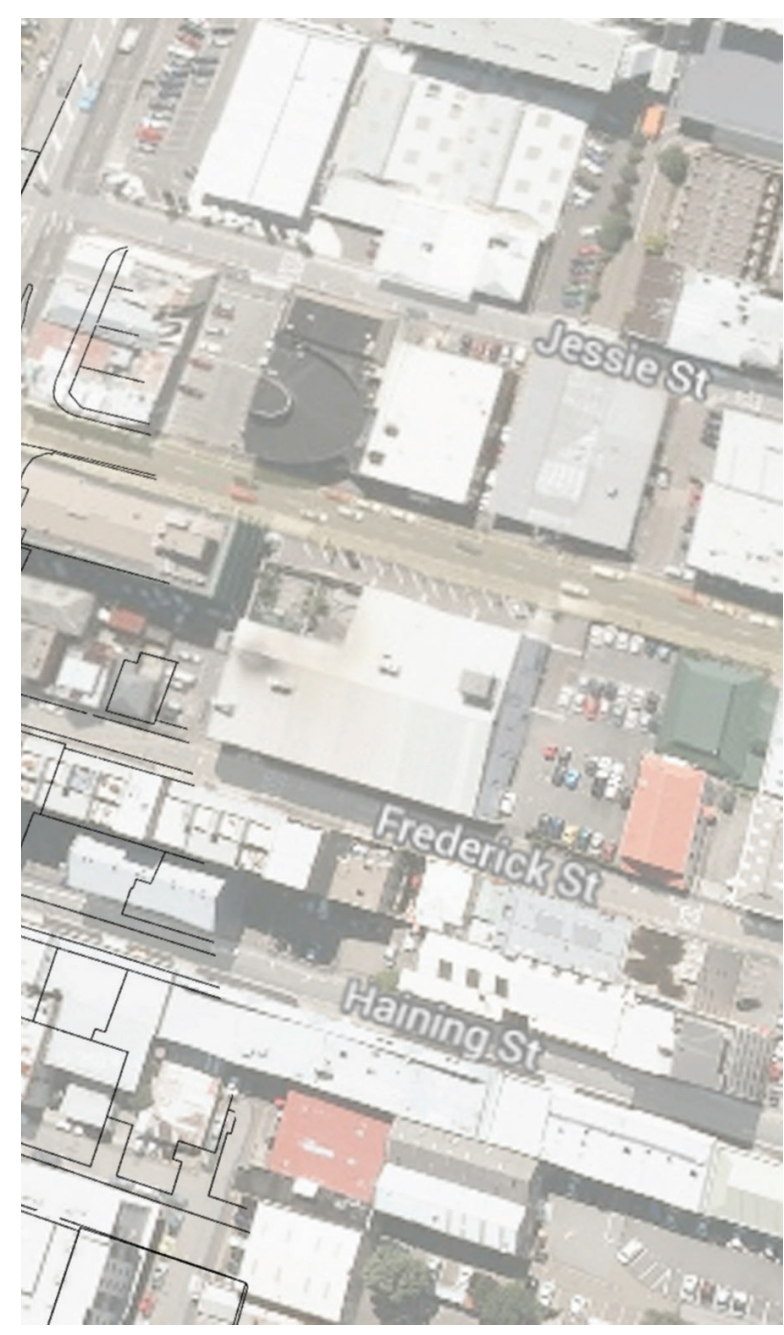

Figure 40. The orange block indicates where the site is in relation to the surroundings

\subsubsection{Site Analysis}

According to the survey on factors most important at present when selecting accommodation, "convenience and distance from campus" was one of the most considerable factors (Thorne, 1992). Therefore, an inner city corner site was chosen at the back of Victoria University of Wellington, Te Aro Campus. The address is 42-44 Abel Smith Street and 37-43 Wigan Street. The site was chosen because of its proximity to the campus as well as the urban context of inner city living in Wellington. 
WIGAN STREET

\subsubsection{Public Sharing: Introducing Community}

This proposal provides 52 single units (approximately 770 habitable rooms per hectare), which are distributed in three building blocks of various heights. The breakdown of the building mass created multiple ground entrances throughout the site and allowed a free and convenient ground level circulation. The planning of multiple routes not only directs the residents to their destinations conveniently, but also offers opportunities for them to explore the space in different paths. The external common spaces were formed in between the building blocks and carried onto the roof tops, allowing residents to have diverse range and quality of gathering spaces. A sense of community living started to be formed through the public shared space in between the individual units and the utilisable roof tops.

Figure 41. Site plan showing the configuration of the three blocks

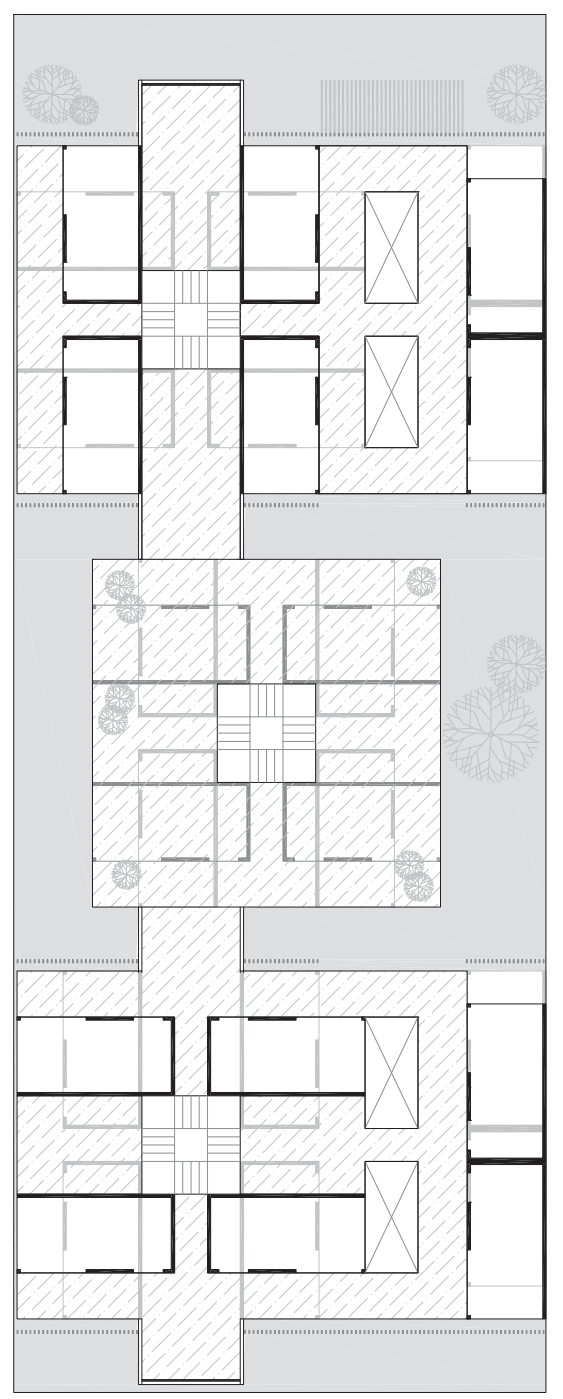

ABEL SMITH STREET 


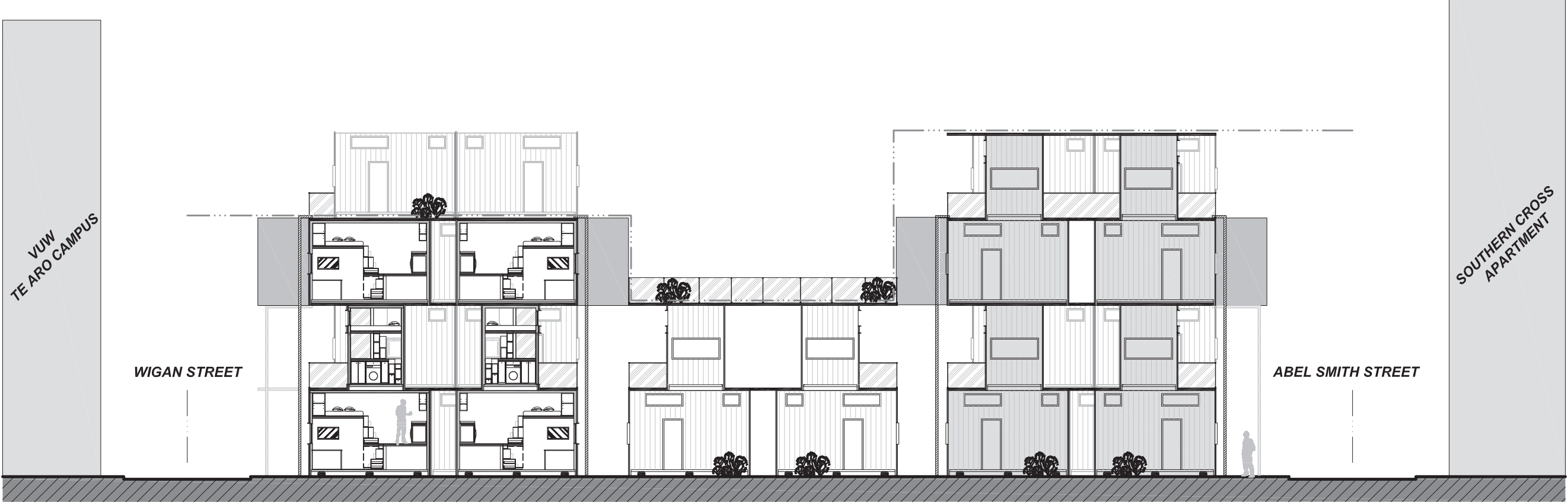

- Figure 42. Longitudinal street section indicating the varying LONGITUDINAL SECTION heights of the three blocks 


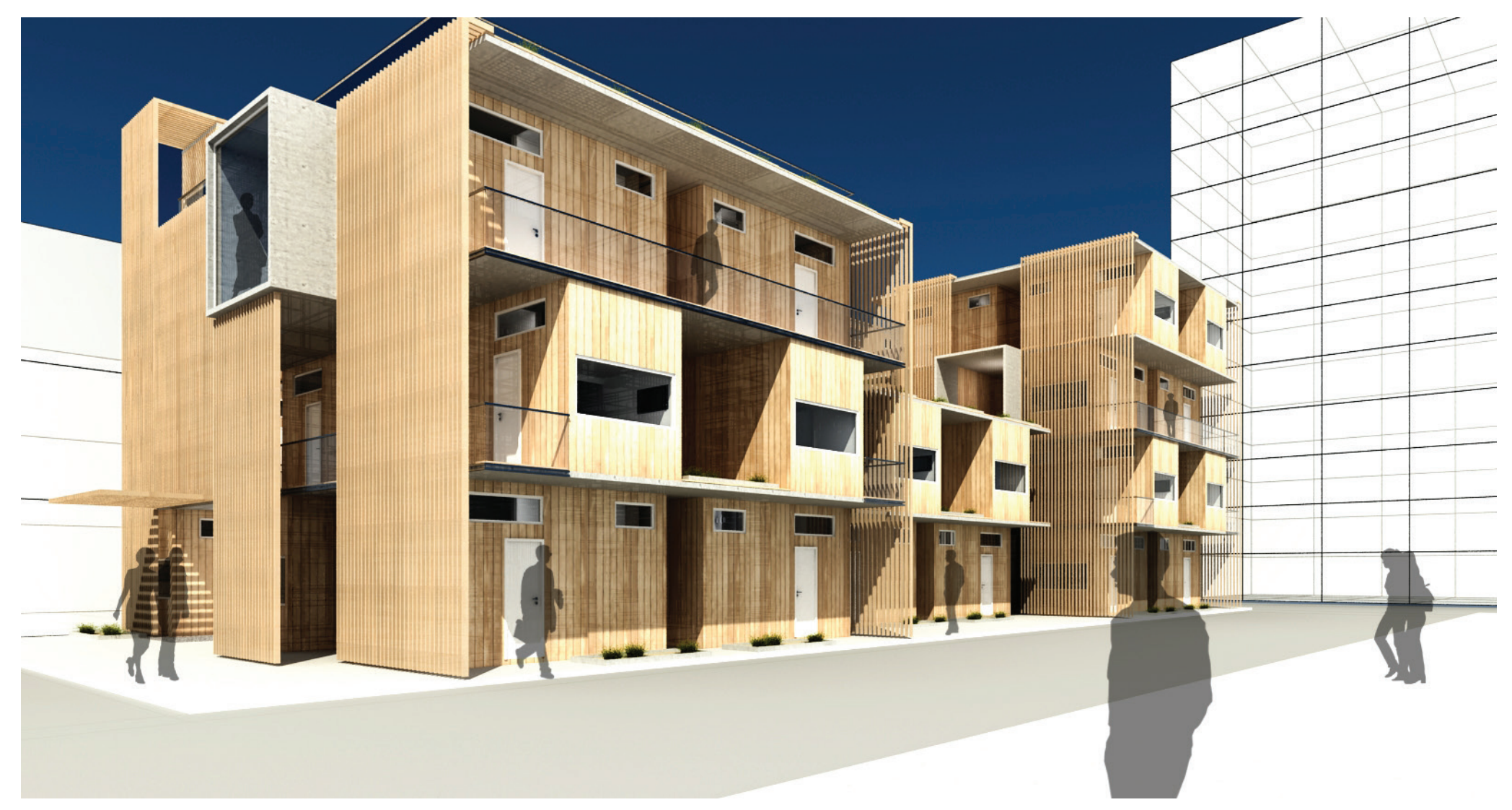


4 Figure 43. Street perspective from Wigan Street corner

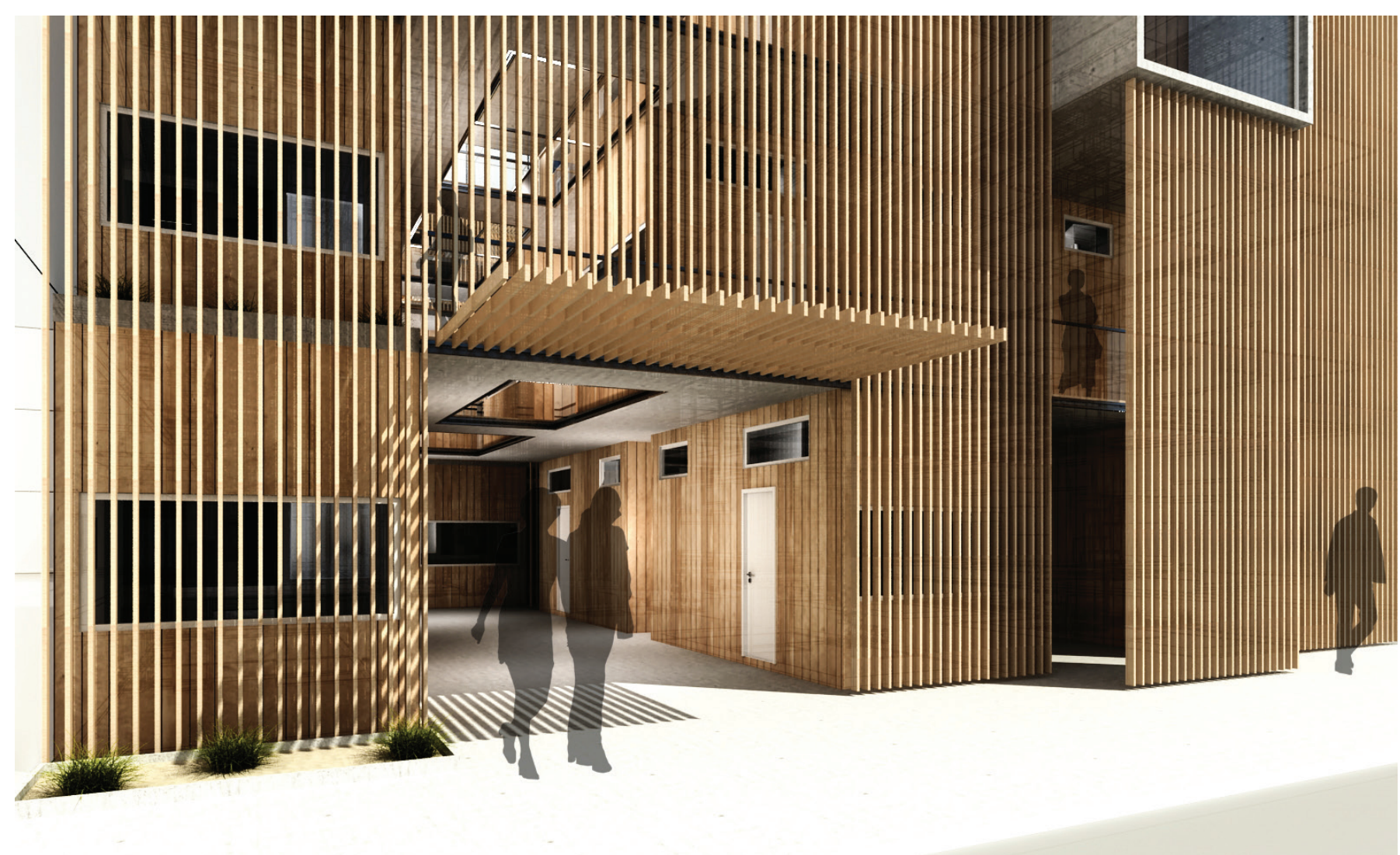

Figure 44. Main
from Wigan Street 

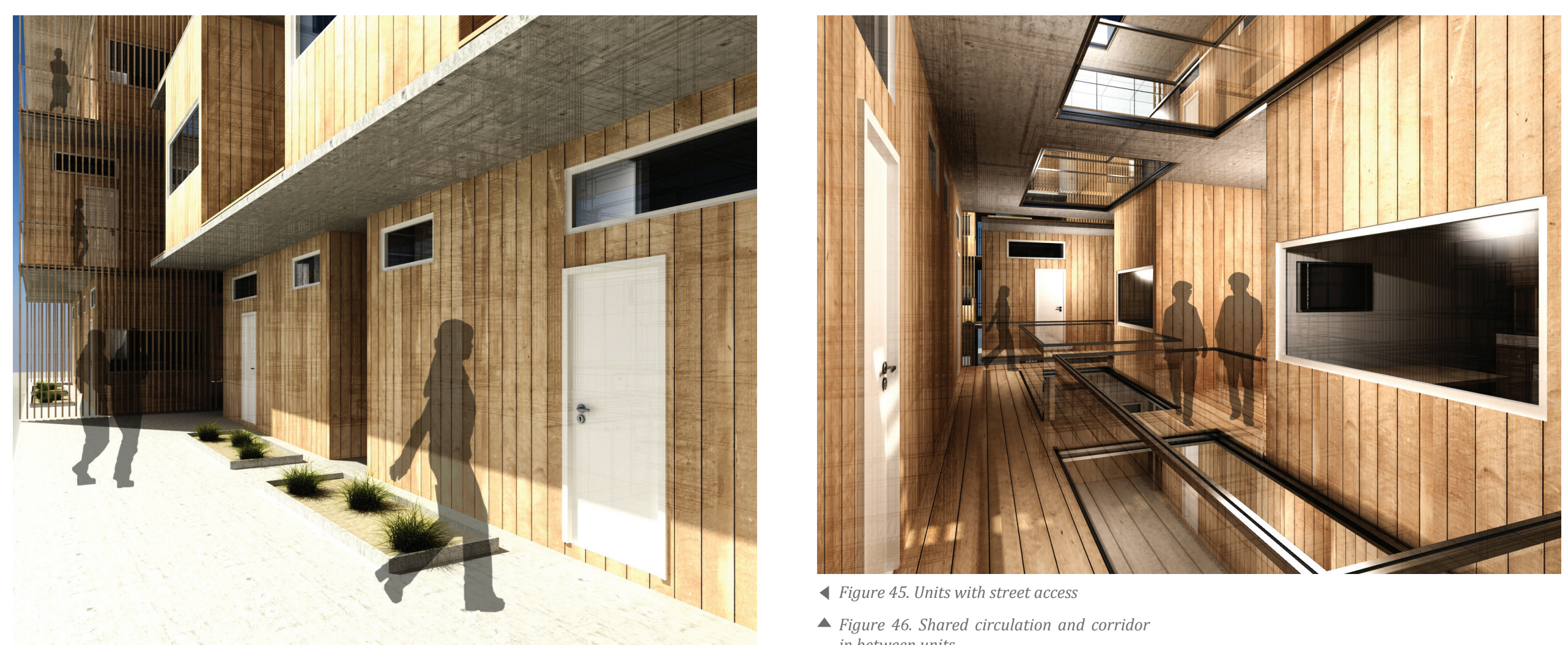

$\checkmark$ Figure 45. Units with street access

- Figure 46. Shared circulation and corridor in between units 


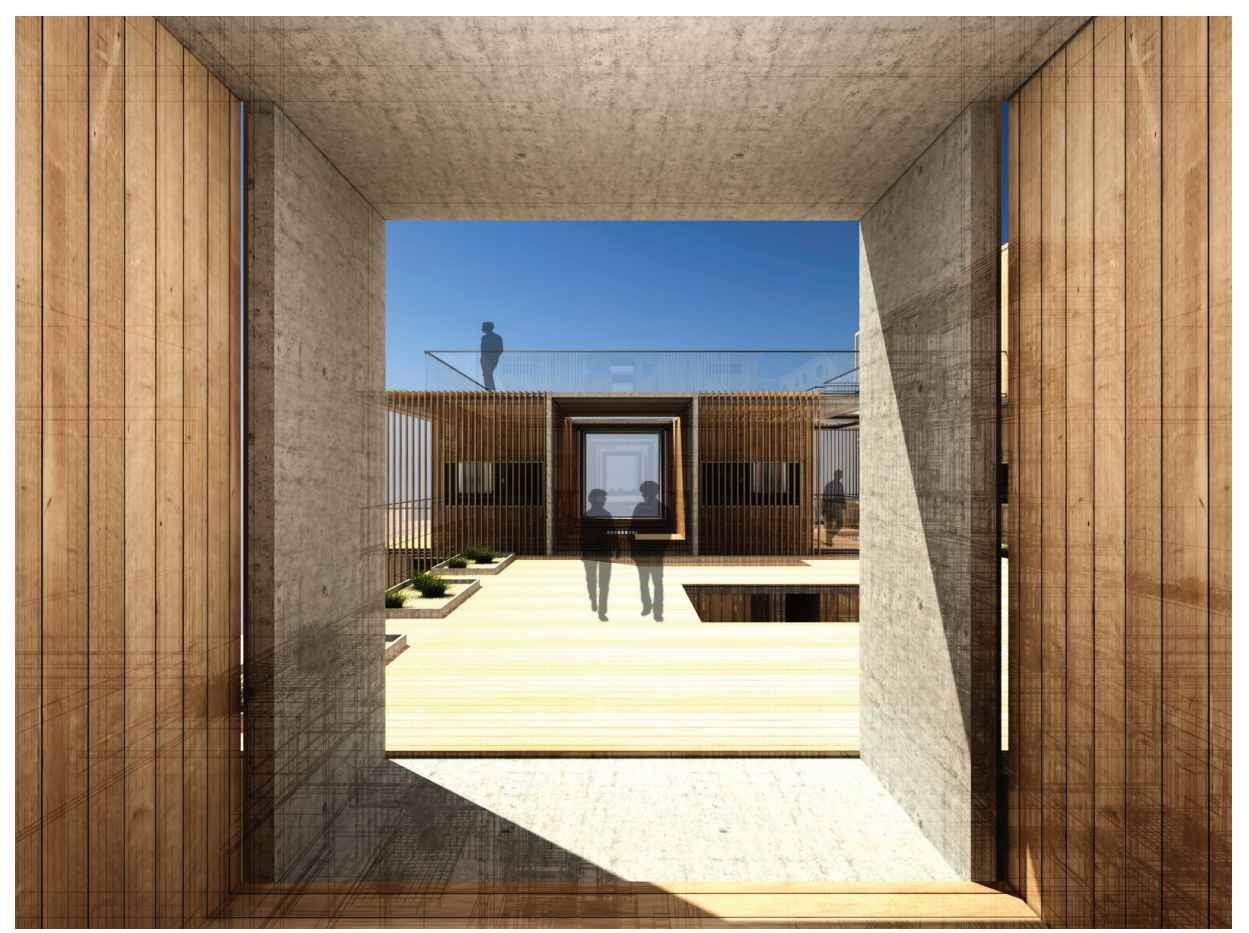

After looking at the quality of life in medium or higher density precedents, the idea of shared space was found to be an important factor in achieving spatial quality in compact living environments. Most of the quality shared and gathering spaces created in this proposal were external, while the internal common spaces were predominately for circulation purposes. Improving the quality of internal circulation and common spaces is a crucial design goal that needs to be achieved in the next step.

- Figure 47. Occupiable rooftop connects all three blocks together as common space for gathering and outdoor activities 
Functional shared space does not need to be physically separate, but can overlap and coexist with other spaces. A positive shared space will be multi-functional. It will give occupants multiple use options, as circulation, a point of outlook, or a place that allows gathering or rest. In the case of compact living, more importantly, it will be treated as an optional expansion of private space.

\subsubsection{New Precedents: Japanese Share House}

Shared housing is one of the rising housing typologies in Japan to address an overwhelming population and land shortage. This particular shared house project, by Naruse Inokuma Architects, has effectivelycreated several efficient shared spaces in between individual units, achieved by carefully planning and enlarging some of the circulation routes. Thereby, the potentialforadiverseinternalsharinginteraction is created between different rooms and levels. 

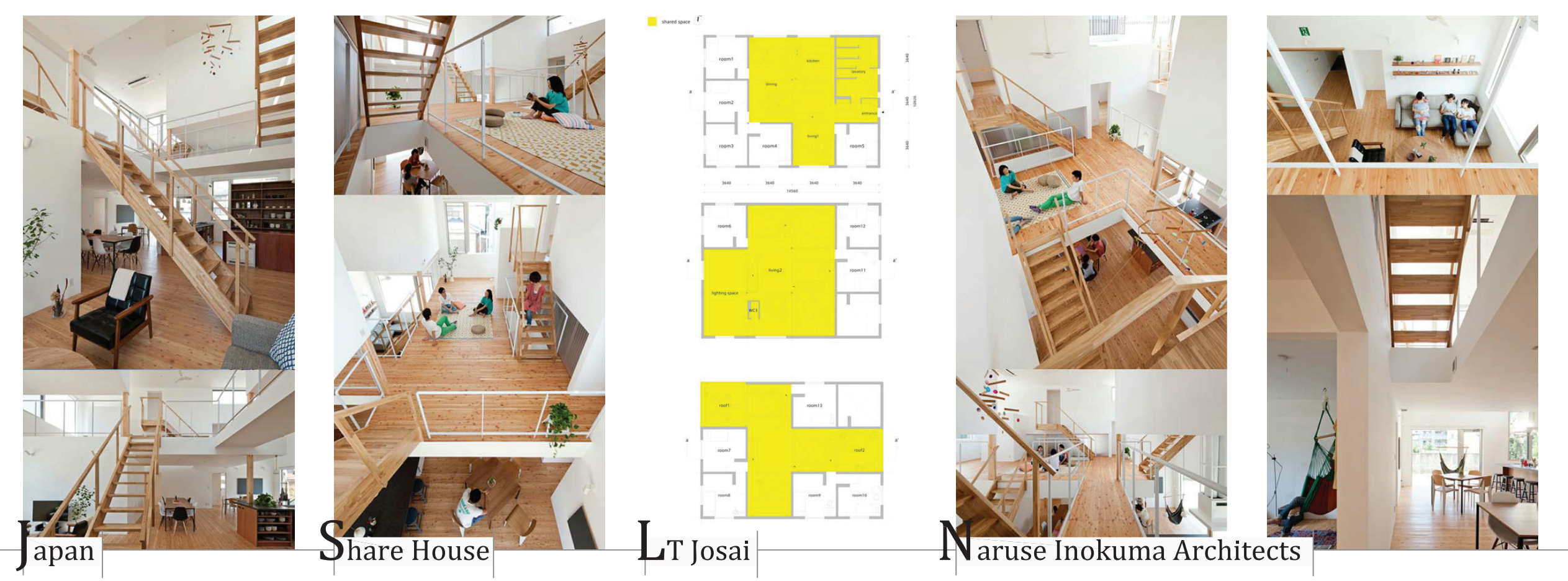

- Figure 48. Shared House by Naruse Inokuma Architects, an example of where

circulation overlap and co-exist with functional shared space among the residents 
The concept of internal shared space was investigated in this iteration to enhance living quality in a medium or higher density student complex. 48 single studios and 10 couple studios were planned and distributed in two identical four-story apartment buildings with a sky bridge and roof terrace connecting them on the first and third floors. The living density in this proposal has increased to 860 habitable rooms per hectare. The new introduced couple studios allowed the apartment to accommodate more residents. Internal shared spaces of various sizes were planned throughout the building surrounded by individual units on each floor. Such partially enlarged circulation space can be occupied as living and dining areas by nearby occupants for occasional events. A large shared living and kitchen located on the ground level with full height transparent windows, allowing visual interactions to the street. A shared laundry was planned on the first level that could be reached conveniently by all the single studio occupants. The top floor contains only couple studios, each with full kitchen facilities so they have less need to travel all the way down to the shared kitchen and living spaces. All units have their own outdoor facing balconies which would not only maximise the amount of day light that could be received, but also break the restricted feeling in compact space by allowing them to have a bit of outdoor living experience. Instead of having a conventional continuous staircase, the vertical circulation was broken into several parts, maximising the chance of spatial exploration by creating interesting visual complexity, facilitating diverse social interactions in between volumes and spaces.

Reflecting on the share house design on the phase two, the balance of public and private space relationships became a crucial factor that affects the overall living quality. In this case, the transition of space between the two was rather straightforward. Although all the units were functioned as individuals, the way they were planned with doors directly facing common shared space makes them feel as if they are bedrooms in a large house. Due to the lack of the sense of ownership of the common space and territories, it may result in underutilised shared spaces. Finding a way to create transitional territories was set as a design goal in the next design investigation. 


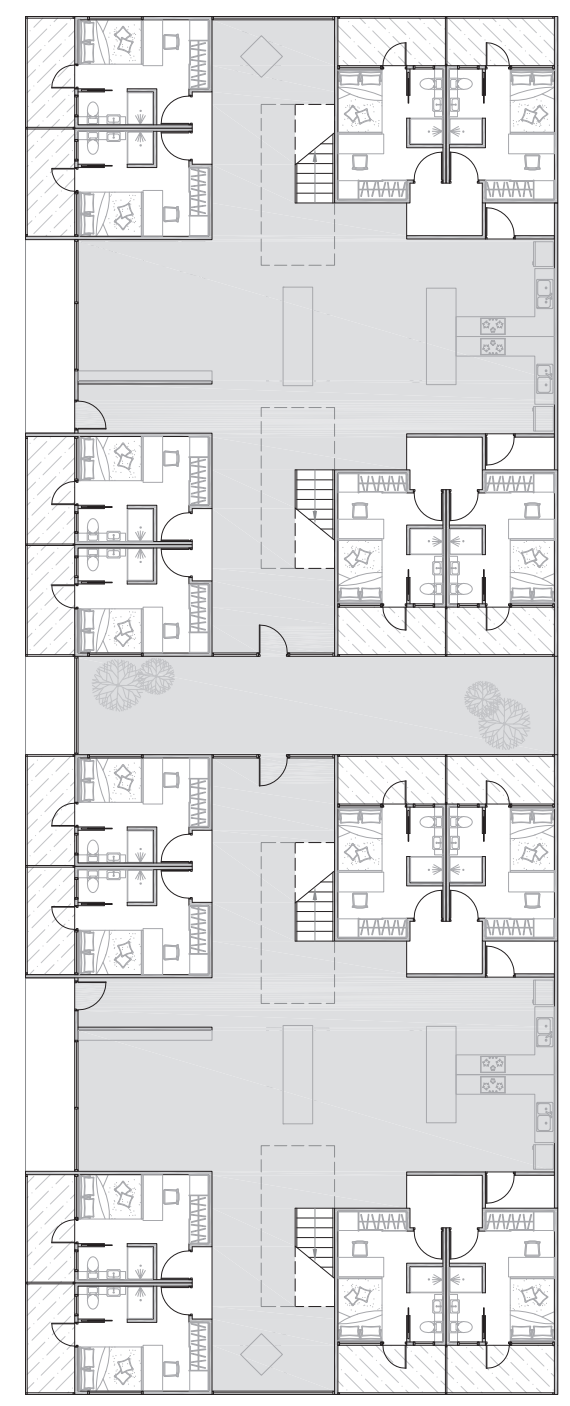

GROUND FLOOR PLAN

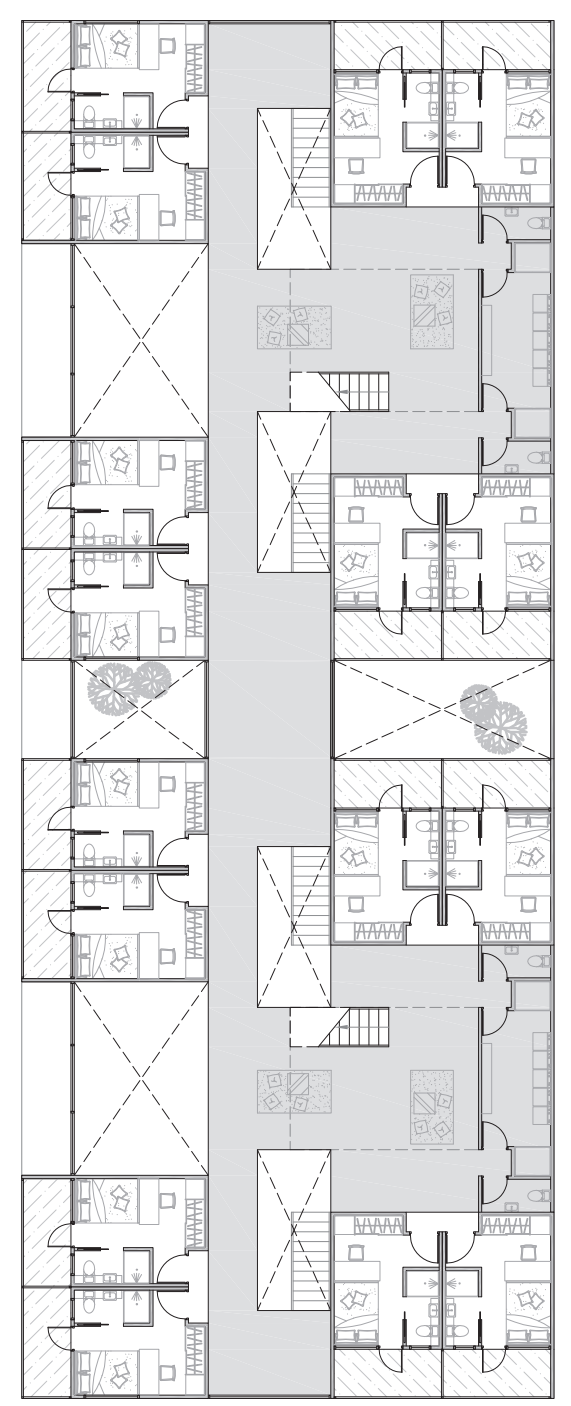

FIRST FLOOR PLAN

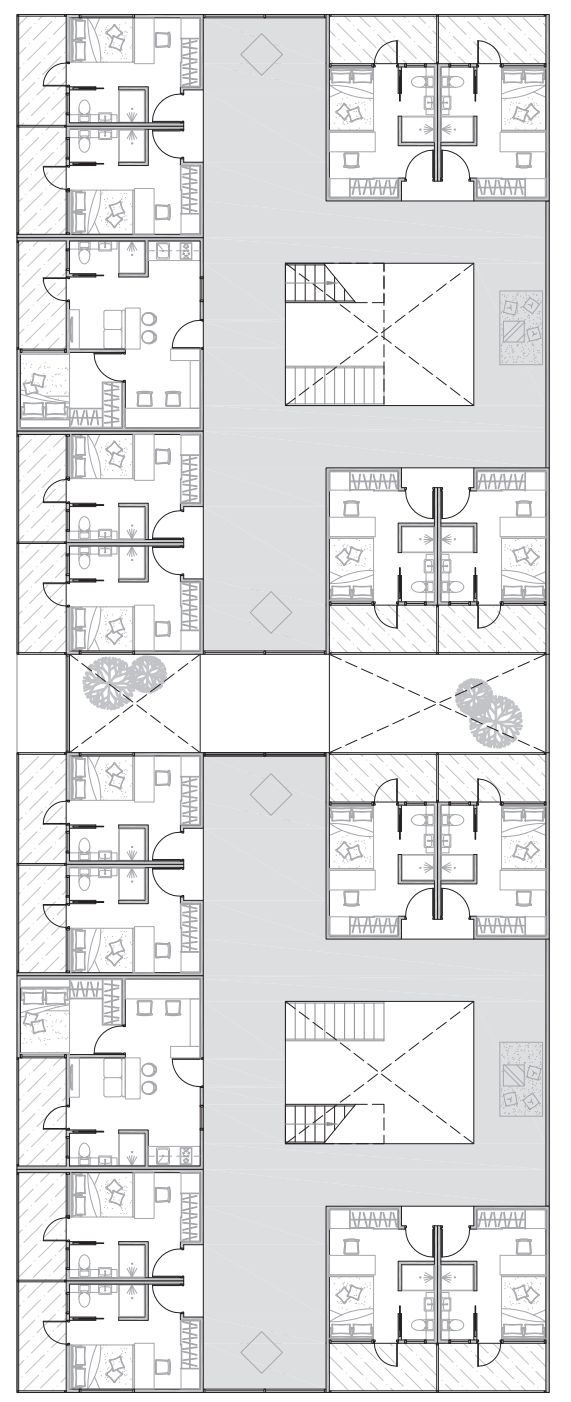

SECOND FLOOR PLAN

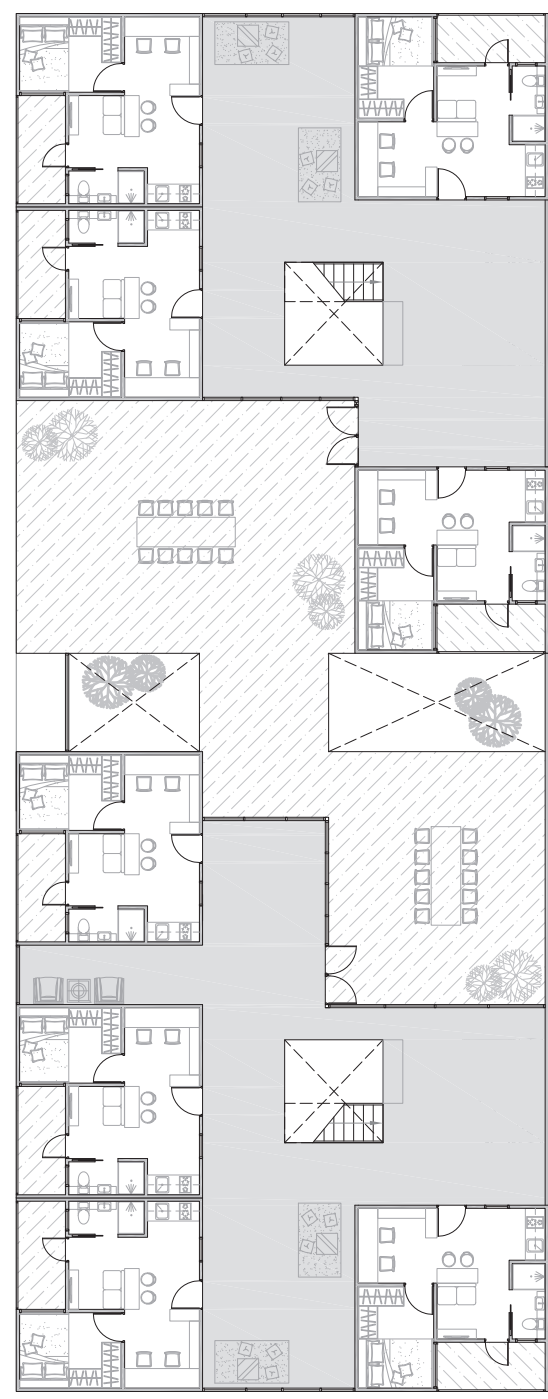

THIRD FLOOR PLAN 
As previously discussed, creating transitional space in between private and common space becomes necessary in share housing. If hyper efficiency was presented as double duty furniture and walls in the interior of the private rooms, then a double duty transitional space in between can be evaluated as a scale extension to the space outside the individual units. Creating this third space to connect the private and public areas would make a positive impact on the livability of minimal living areas.

\subsubsection{Private within Public: Continuous \\ Circulation Loop}

Entering from a middle courtyard, the new design contains 56 units with three different modules for 86 residents and achieves approximately
980 habitable rooms per hectare. Functioning as a series of communities within a community, the layout shows how refined transitional spaces are now allocated for each individual unit so they would each have equal occupiable shared space adjacent to their units. A strong visual cross interaction was created with the public and shared space circulating through multiple split levels. The vertical circulation has been modified from conventional staircase to a continuous loop that goes all the way to the top with transitional turning platforms. This split level circulation loop has also accentuated the sense of semiprivate territories by visually separating the transitional shared spaces into different floor levels. The idea of transition carries into each private unit by entering from a private balcony, and creating a distinct compact living experience.
Large group events can be held in a spacious common living, dining and study area located on the ground floor. Extra courtyard spaces were created by setting the building boundary 3 meters from the neighbour. Commercial spaces were introduced at the edge on ground level, allowing them to be leased as café or restaurants in this high demanded commercial environment.
Figure 50. Shared House II unit types in relation to split level circulation

$\nabla$ Figure 51. Shared House II floor plans 

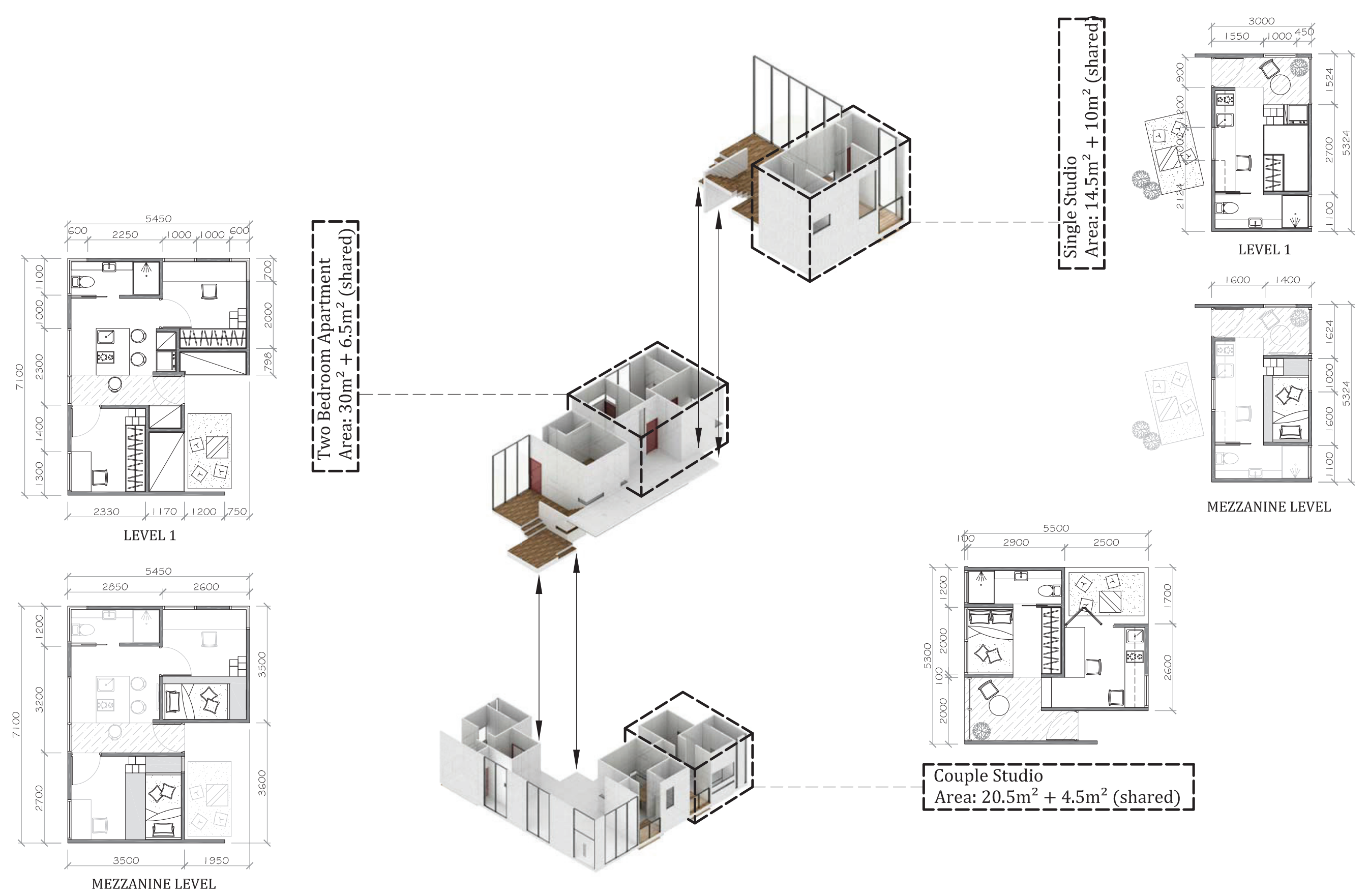

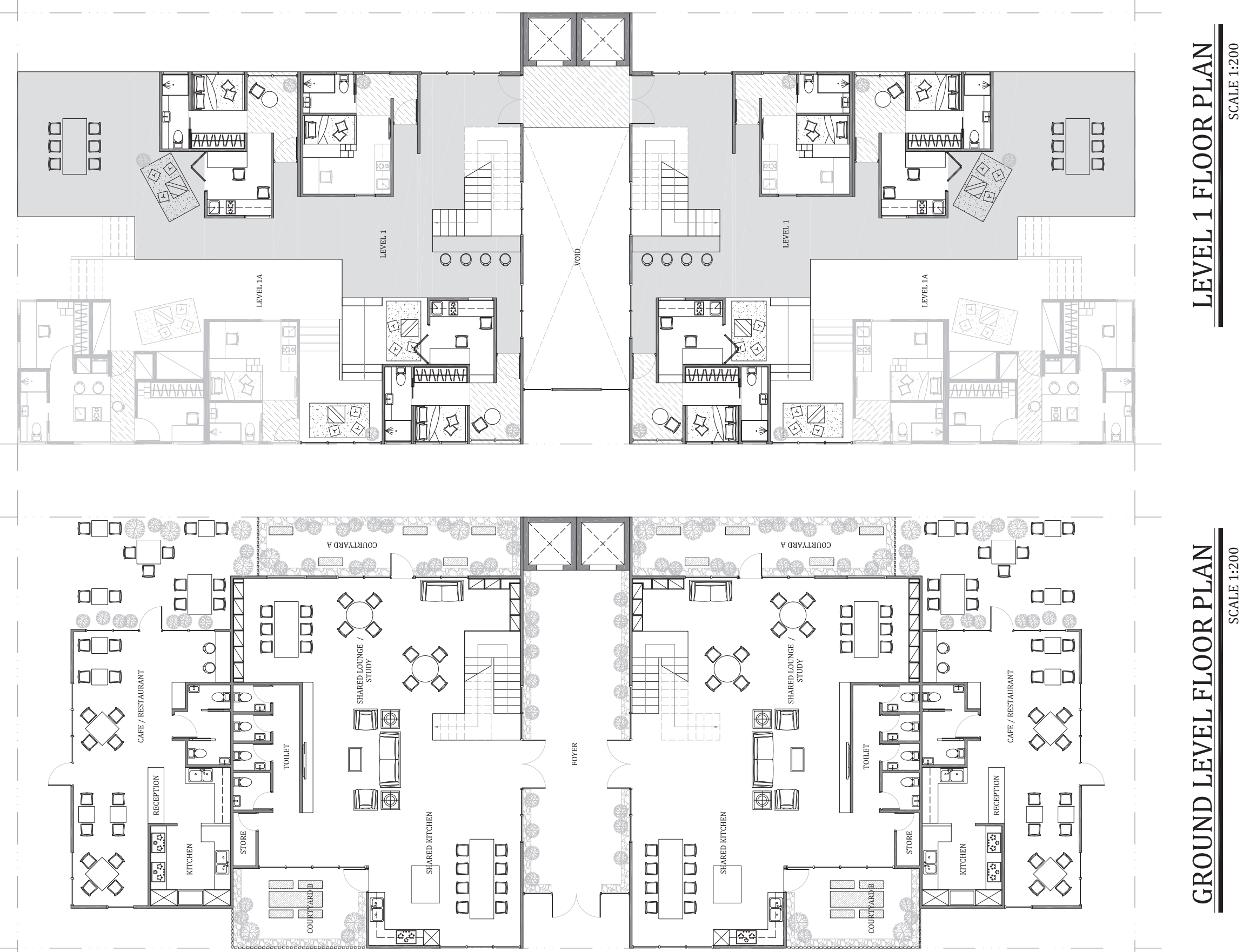

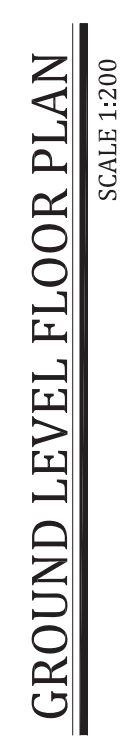



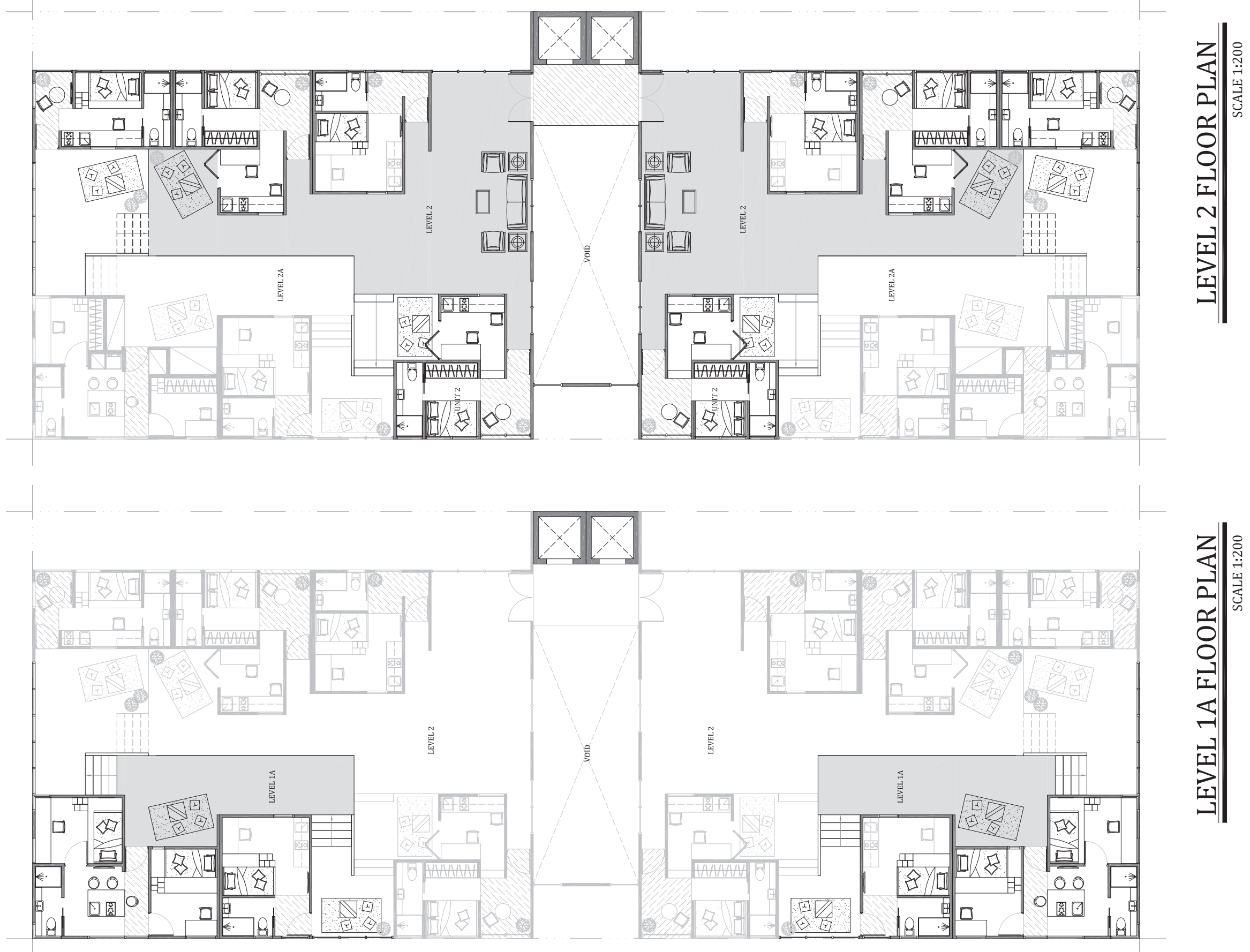
In the words of Dorgan and Evan (2008), considerations of a high quality design encompass "proportion, sense of identity, size and rhyhms of openings, circulation, access to light and air...and suitable for the activities that take places in them" (p. 149). This design iteration has addressed those aspects comprehensively. The split level apartments create a distinct pattern of window openings. By introducing the split level continuous circulation loop, the design has suggested and created a new apartment typology. The vertical circulation is no longer constrained by staircases, and the floor levels were transformed into various transitional split platforms. These platforms are planned with full height window which allow the internal common space to have good "access to light and air". The conventional corridor circulation in an apartment living environment has become spacious shared space that serves multiple purposes. Such shared space allows occupants to extend their private space to the common area, strengthens the neighbourhood relationship as a community. This new shared circulation typology interprets a way of providing suitable space for the "activities that take places in them".

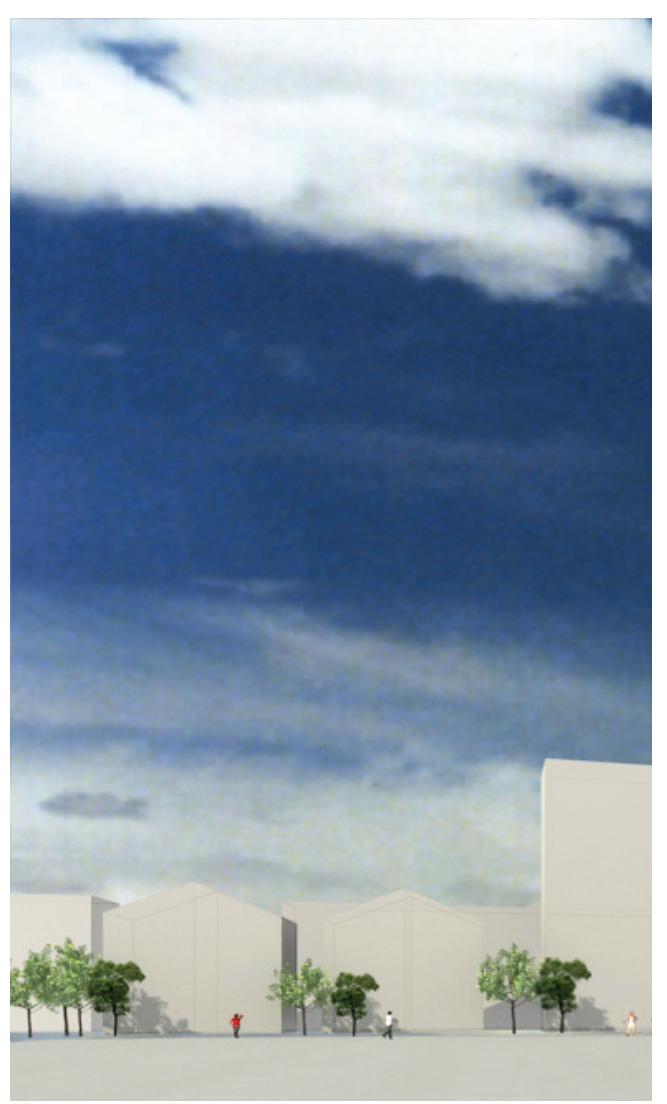




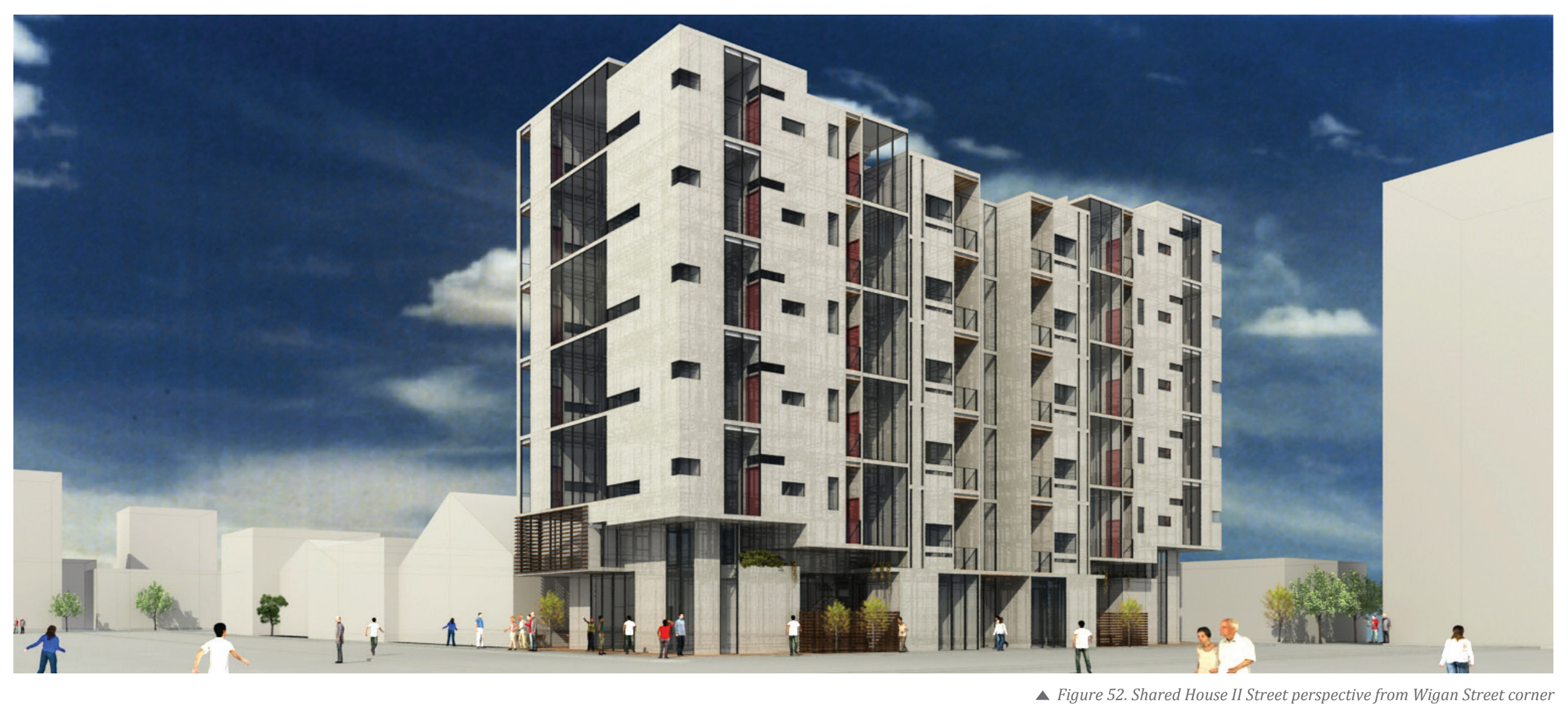




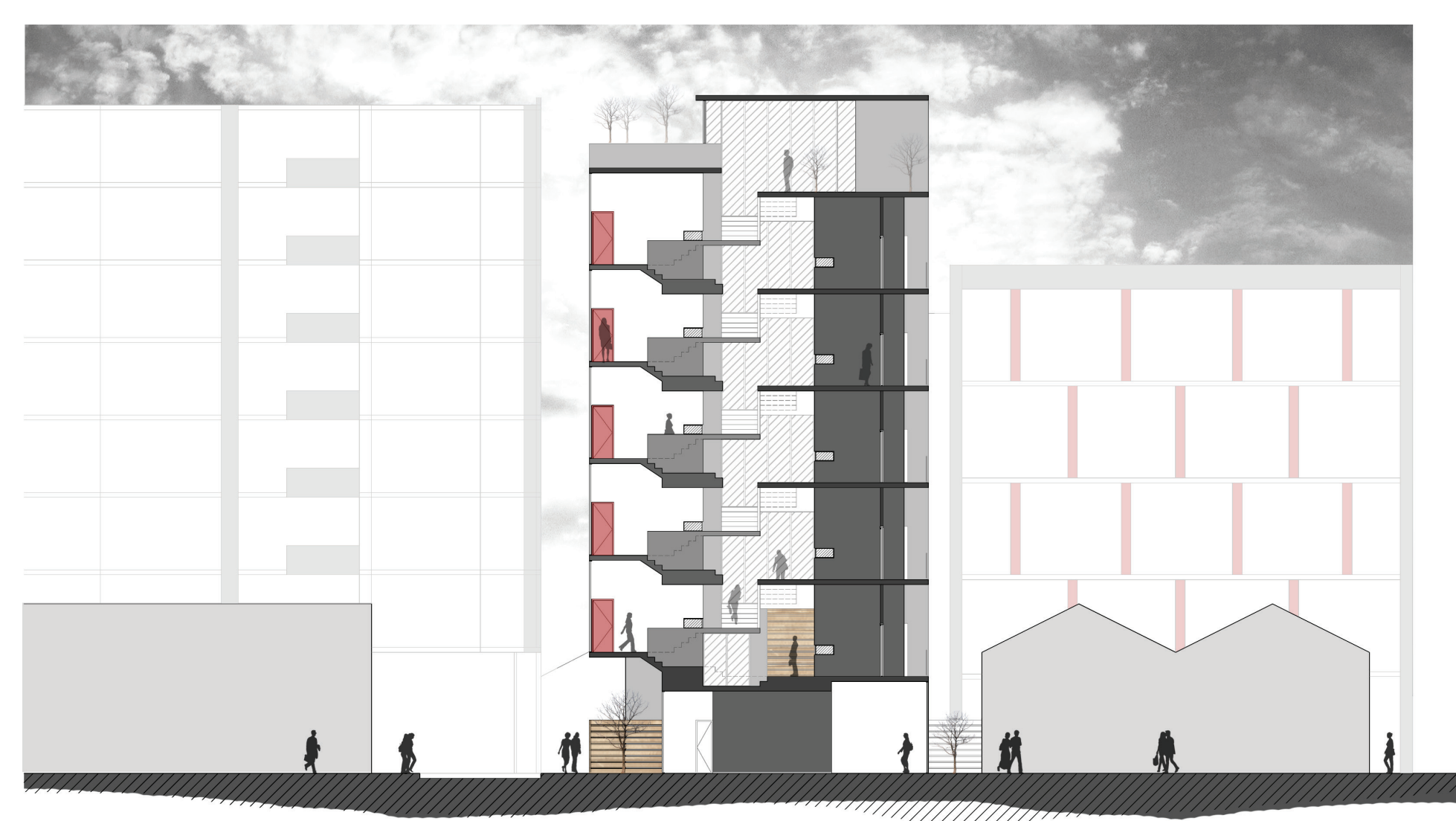

- Figure 53. Shared House II street sections from Abel Smith Street 


\subsection{FINDINGS AND REFLECTIONS}

Shifting from the interior hyper efficiency of individual units to efficient internal spatial relationships between multiple units, the investigation in this chapter has tested and argued that double duty circulation spaces play a crucial role in terms of improving the quality of life in compact apartment living environments The idea of making limited space available for more than a single task allows the space to be utilised for different purposes, as per the needs of occupants. The shared house design iterations have shown how horizontal circulation can function as an extension of private space rather than just as a linkage from one place to another, and that vertical circulation might not necessarily be constrained by conventional flat floor levels connected with staircases. Creating such internal spatial complexity would form individual transitional territories between the public circulation and private units. Unlike the isolated living in conventional apartments, shared housing provides the chance for the occupants to utilise common areas, gather, and communicate with neighbours in a much more casual and comfortable way. The design investigations have been dealing with the balance of public and private territories and the relationship between inhabitable space and occupants. The intention of the next stage is to extend these factors in the wider site context and look at the relationship between large scales multiple student housing apartments and the balance between residents and general public. 


\section{DESIGN PHASE III: BLURRING THE BOUNDARY}

The final stage of this design thesis extended the scale into an urban block and investigated ways of connecting several shared housing blocks together, as well as pushing the sharing boundary from among the residents only to an aspect of interacting with the general public. Unlike China or other densely populated Southeast Asian countries, most dense inner city apartment designs in New Zealand are focused on single towers rather than a group of buildings. Having buildings grouped together would create opportunities to plan semi-public space that functions for both the residents and the general public, allowing the residents to have a safe territory and on-site shared leisure facilities to utilise. Most examples of such group buildings in China for instance, appear to be enclosed and secured by a property management agency. In most cases, they put up clear defined boundaries such as fence, wall or natural feature entirely surrounding the site with restricted access to separate the common shared territory and the street. This type of community is known as a "gated community". Gated community typologies can be found in many highpopulation cities other than Asia. Back in 2002,
Ed Blackely claimed that about $40 \%$ of new homes in California were behind walls (Nasser, 2002). There are suburban gated community examples in New Zealand too, such as upmarket coastal sub-divisions in Gulf Harbour, Auckland ("Property Problems", 2004). The crime rate may not be a serious threat in New Zealand so the degree of "gatedness" can be different. The final design iteration demonstrated that it is possible to have a degree of "shareness" instead of a fully restricted environment as a possible evolution of a gated student community typology for New Zealand. 


\subsection{TE ARO VILLAGE: SHARING IN PUBLIC}

"In meeting today's high standards, campus housing must address a wide range of concerns from the evolving nature of student life in the age of instant communication to the timeless issues of community and privacy. Contemporary cultural and ethical values, such as social diversity, gender neutrality, and concern for the environment, are key influences on student attitudes and surely will influence how students live in common".

---- Neuman and Ap (2013)
Te Aro Village, the final design investigation, addressed the possibility of expanding the shared space from a restricted internal environment to the external urban surroundings. Having spaces that could be utilised and shared by both residents and the general public illustrated a different way of addressing the issue of community and privacy in inner city living. The significance of creating transitional space or the third space in between apartment buildings, leisure facilities, commercial spaces and public areas has been demonstrated, achieving high density housing in an open and friendly way. Open and friendly in this case means the design allows the residents to be able to get away from a sense of isolation in conventional apartment living environments, and enjoy open space and shared leisure facilities with neighbours in various degrees of both visual and physical interactions with the general public. 


\subsubsection{Site Extension}

The previous site for the shared houses in design phase two was chosen for the final design iterations, with a site expansion towards Taranaki Street. The new site is approximately four times larger than its original. The expansion tested the needs of community living in between several apartments and the relationship to public facilities and spaces. In order to demonstrate the ways of forming such shared relationship, the decision was made to retain the newly constructed Light House Cinema on site and allow it to function together with the village.

The surrounding sites have a height limit of 27 meters according to the current district plan, and new buildings nearby have been intensified to the $27 \mathrm{~m}$ limit. Therefore it is fair to predict that the proposed student village will be sit within a densely built environment in the future. 


\section{Light House Cinema}

The stylish Light House Cinema is proposed to remain on site and function together with the new student village.

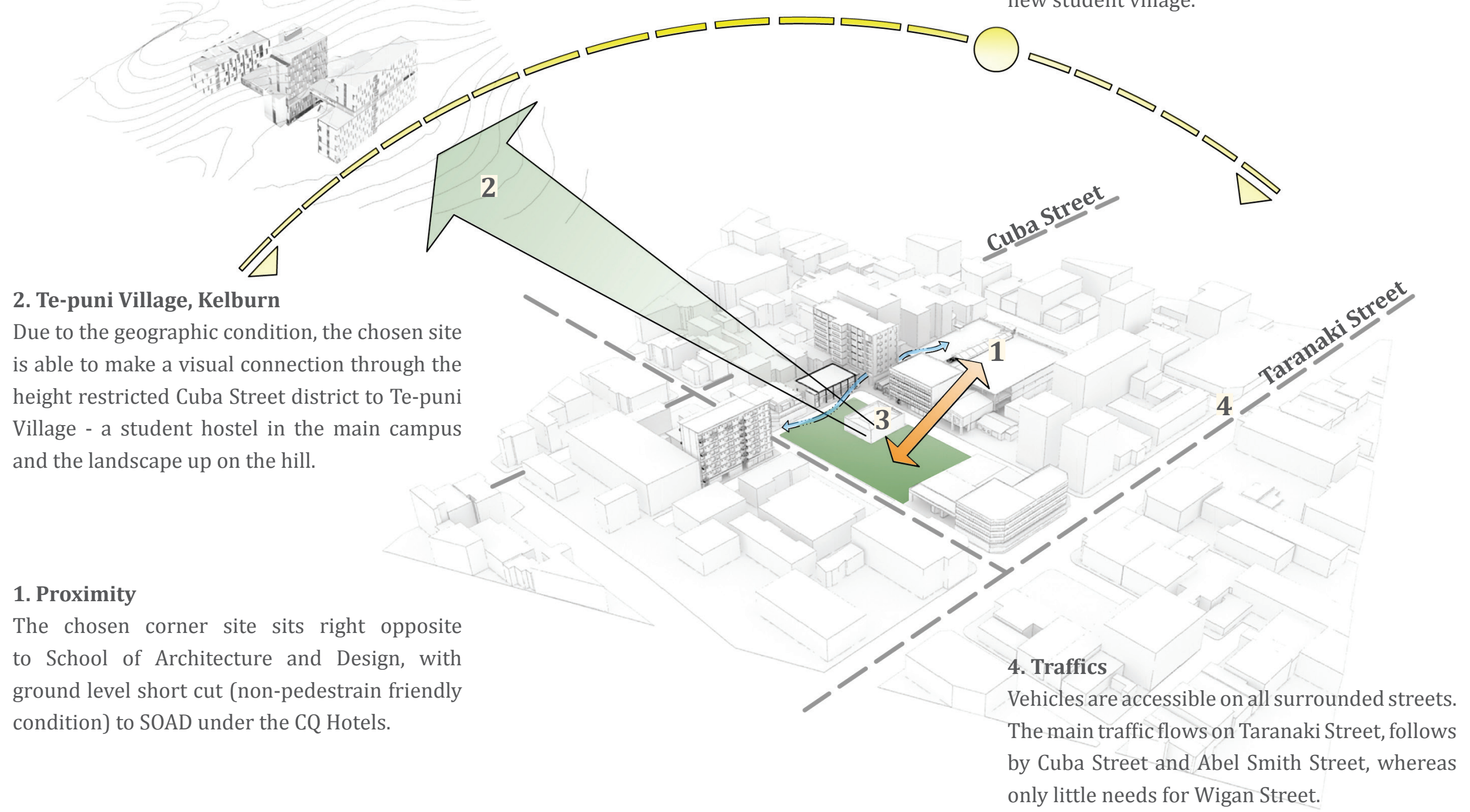




\subsubsection{Recessed Boundary}

The design began by looking at relationships between private space around the house and public streets. As the diagram in figure 55 demonstrates, safety and sense of ownership is lost in a space without a defined boundary. In the case of a gated community, a fenced space with clear defined ownership blocks out the sense of sharing and results in the private space being isolated. In order to introduce shareness in gated community typology, the design proposed to look at a solution in between the two extreme examples. Recessed space within the boundary allows visual interactions between the private and public yet maintains the sense of ownership and territories. Instead of totally separating the relation between pedestrians and residents with a fence, lowering the site half a floor below the street level will achieve the same safety concerns in a more friendly way, and avoid the private inhabitable space being isolated. 

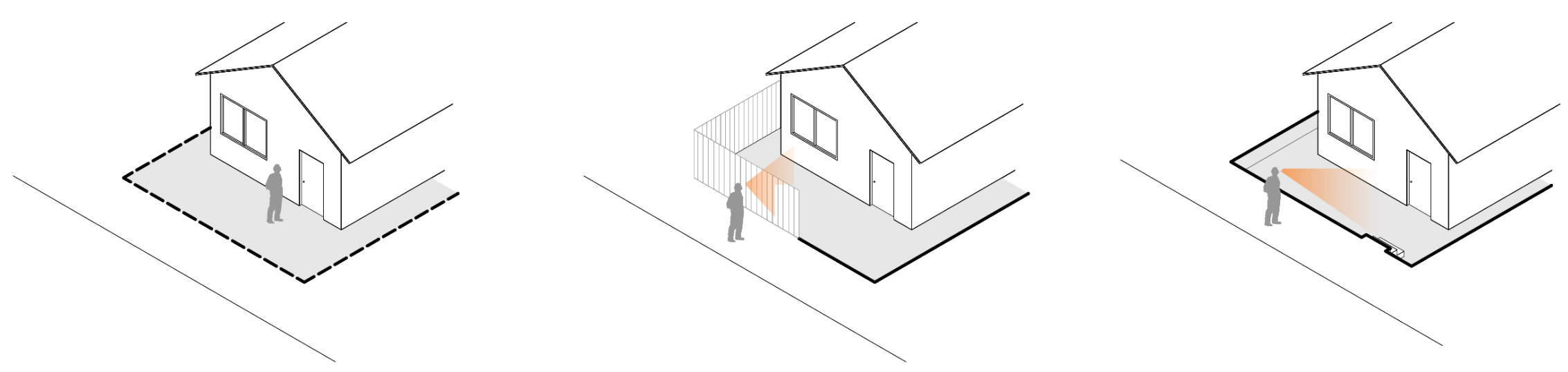

- Figure 55. Relationships between public and private occupants in different boundary conditions

$\overline{71}$ 


\subsubsection{Negative Space}

The second step was to test the potential configurations of a group of apartments through a series of form iterations generated by different strategies. In consideration of the site conditions and the balance between density and living quality, the negative module was picked to be developed further because of the opportunities to utilise the spaces created in between the blocks.

Figure 56. Form generating process with series of iterations driven by different strategies 

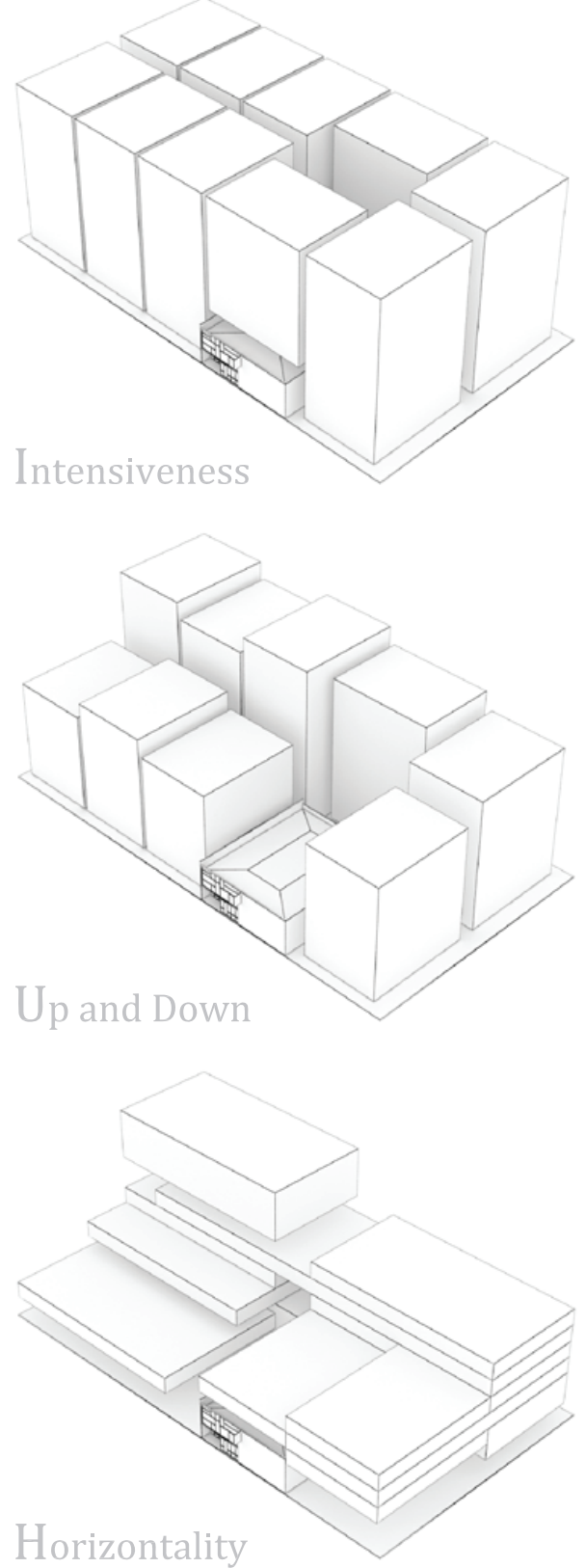
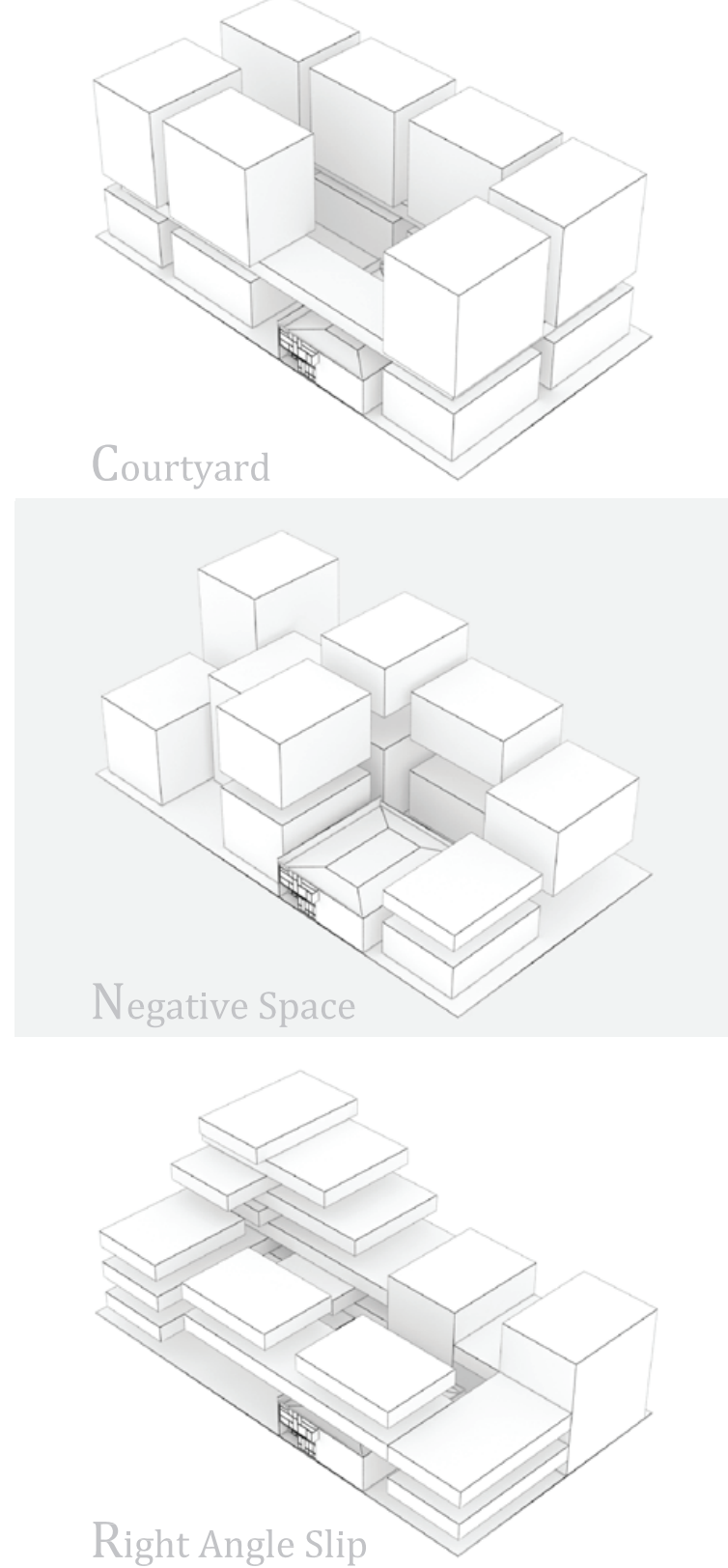
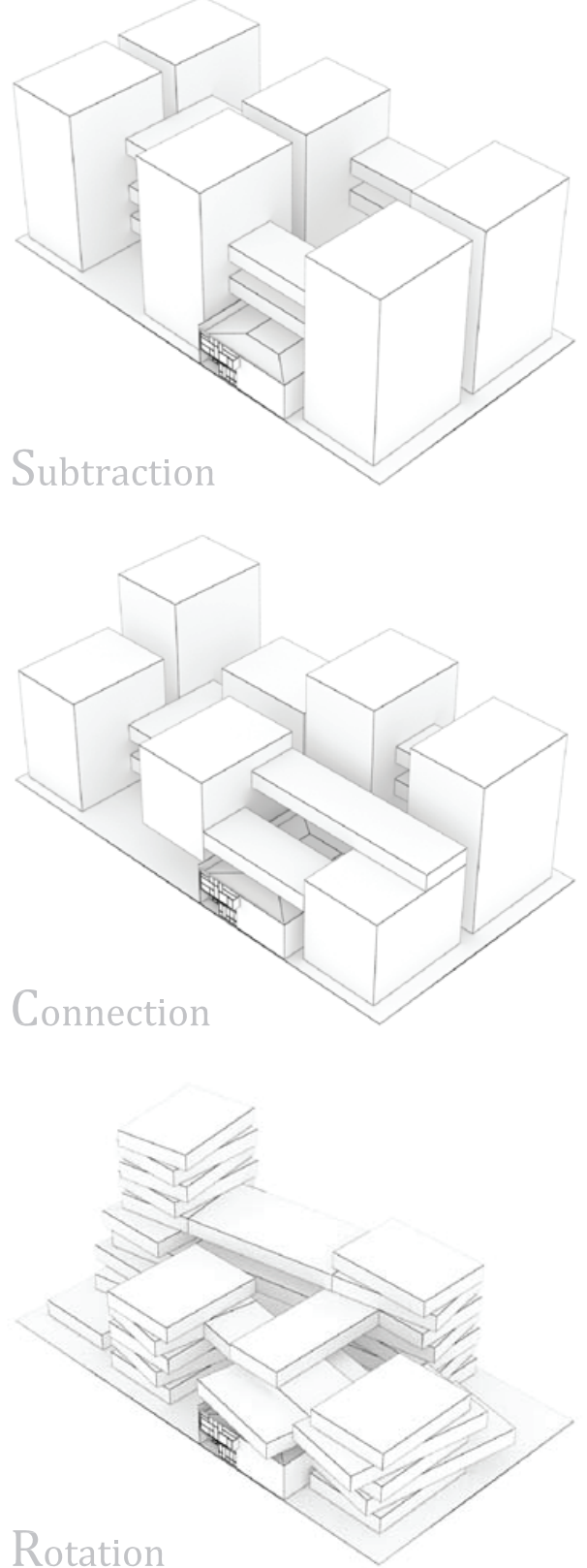


\subsubsection{Design Proposal}

In response to the sun and site conditions, the northern corner was decided to be kept lower to allow sun light to the entire site. The five proposed apartment towers had the same intention; to generate views and light within by having various heights. An urban courtyard was formed in the middle of the site as shared space with the public, providing access and linking Abel Smith Street and Wigan Street with a diagonal short cut. As previously discussed, it is likely to see the site would be surrounded by high-rise buildings in the future, which made the public share space introduced in this proposal a crucial aspect in creating better living quality in a densely built environment.

Figure 57. Birdeye perspective from the corner of Wigan Street, overlooking the roof terraces in different levels as well as the visually shared landscape around the site. 


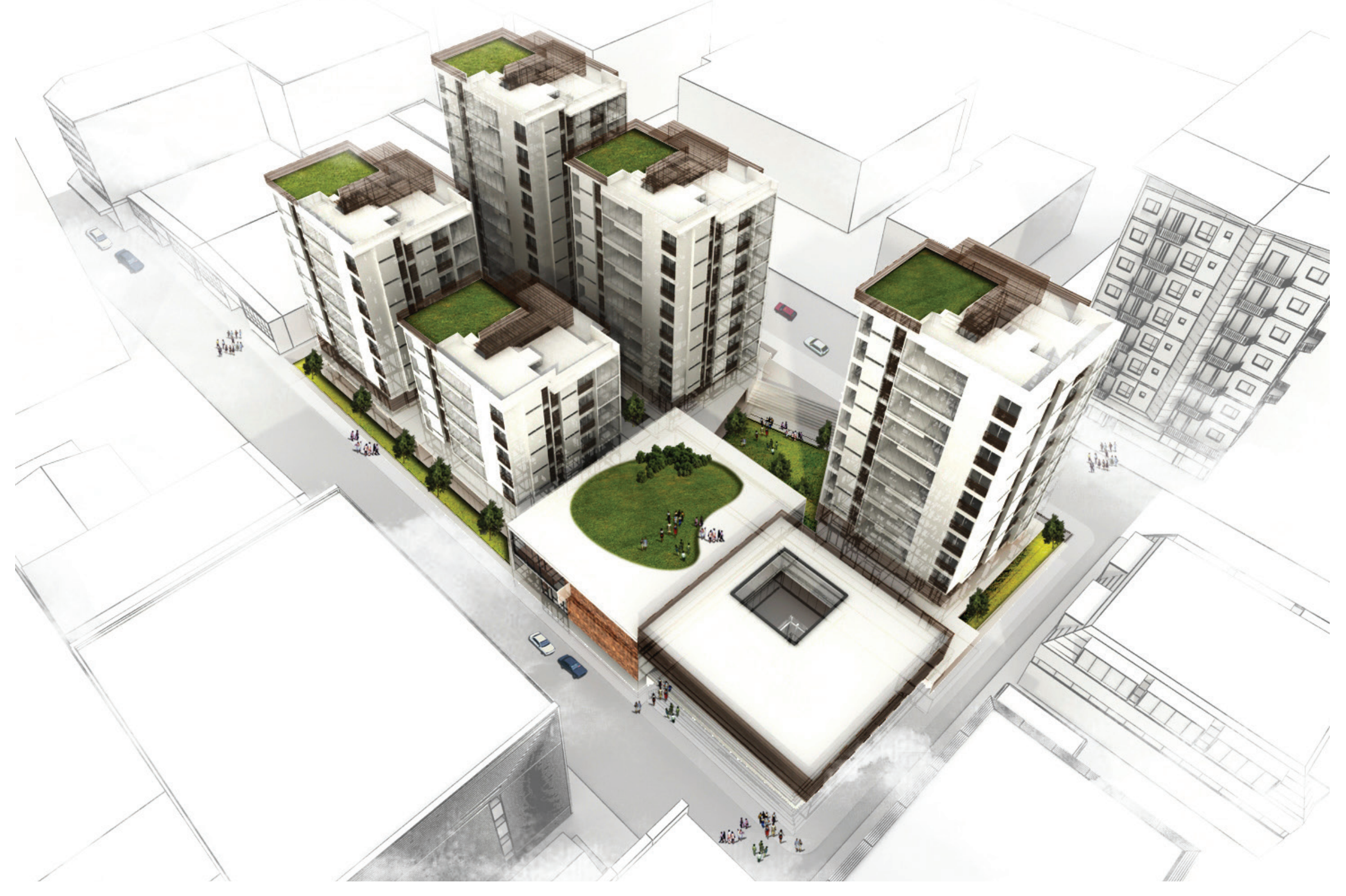


All five apartment towers are located on the recessed ground level with transitional setback space from the site boundary. The recessed setback space kept the public pedestrians away from the buildings but they can stil visually enjoy the landscape within. Public access is created at both sides, leading to the exhibition space on the corner of Wigan Street and the public park at the back of Light House Cinema. Restricted entrances to the apartments were placed on two sides of the public shared spaces. One was for entering Tower A, and the other formed an enclosed private territory in between the rest of the four towers. Major onsite shared leisure facilities were planned in this private territory, which contains two common study areas under Tower D and E, as well as a gym under Tower C. Café and restaurants were placed under Tower B with the opening to the park in order to make the park become a more friendly inhabitable space to the public. A secondary private circulation that linked between all five towers was introduced on the first floor. Running horizontally across the entire site, this external connected platform created a degree of sharedness among all residents, as well as increasing the visual richness by having the interactions with the park on the ground floor. A cantilevered function room was located at the Wigan Street corner above the ground floor exhibition space, which can be used for university events as well as hired by students for occasional functions. A new rooftop structure was proposed for the Light House cinema to transform it into a north facing inhabitable roof terrace that could be shared by the residents. 


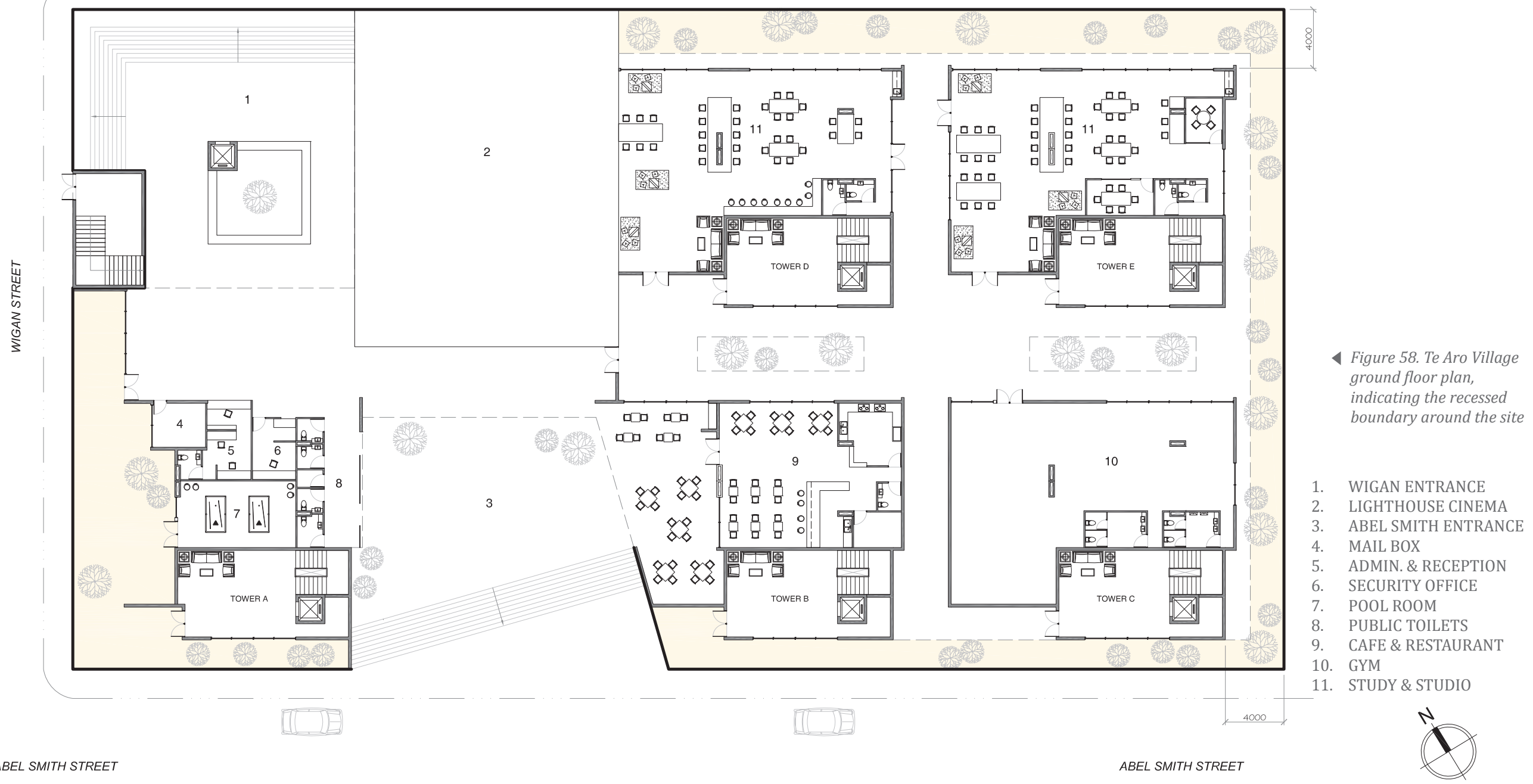




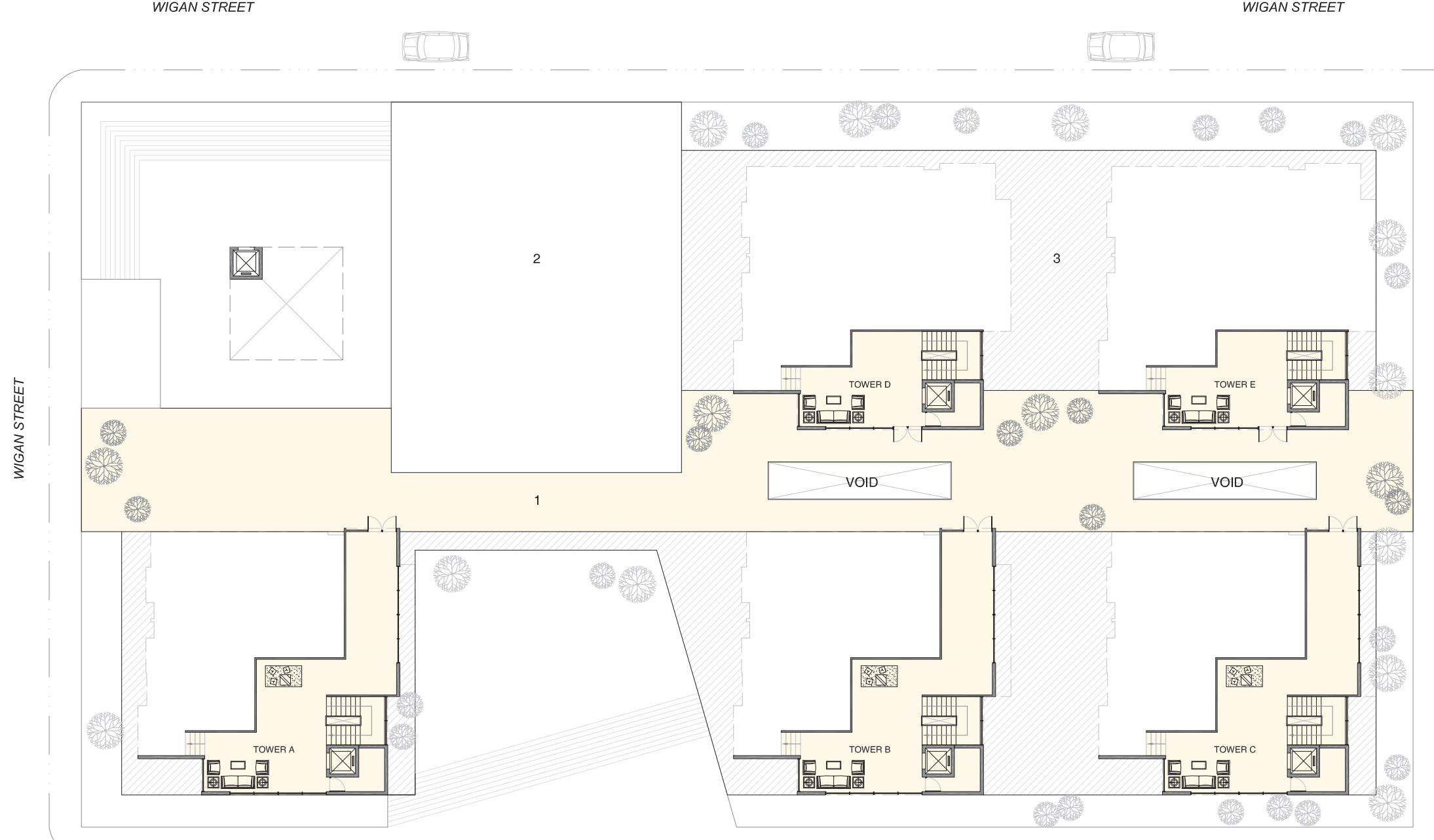

4 Figure 59. Te Aro Village first floor plan, indicating the secondary outdoor private circulation

platform that connects all five apartment towers

- Figure 60. Te Aro Village apartment plans

PRIVATE CIRCULATION PARK

LIGHTHOUSE CINEMA 3. GROUND FLOOR ROOF

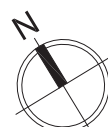



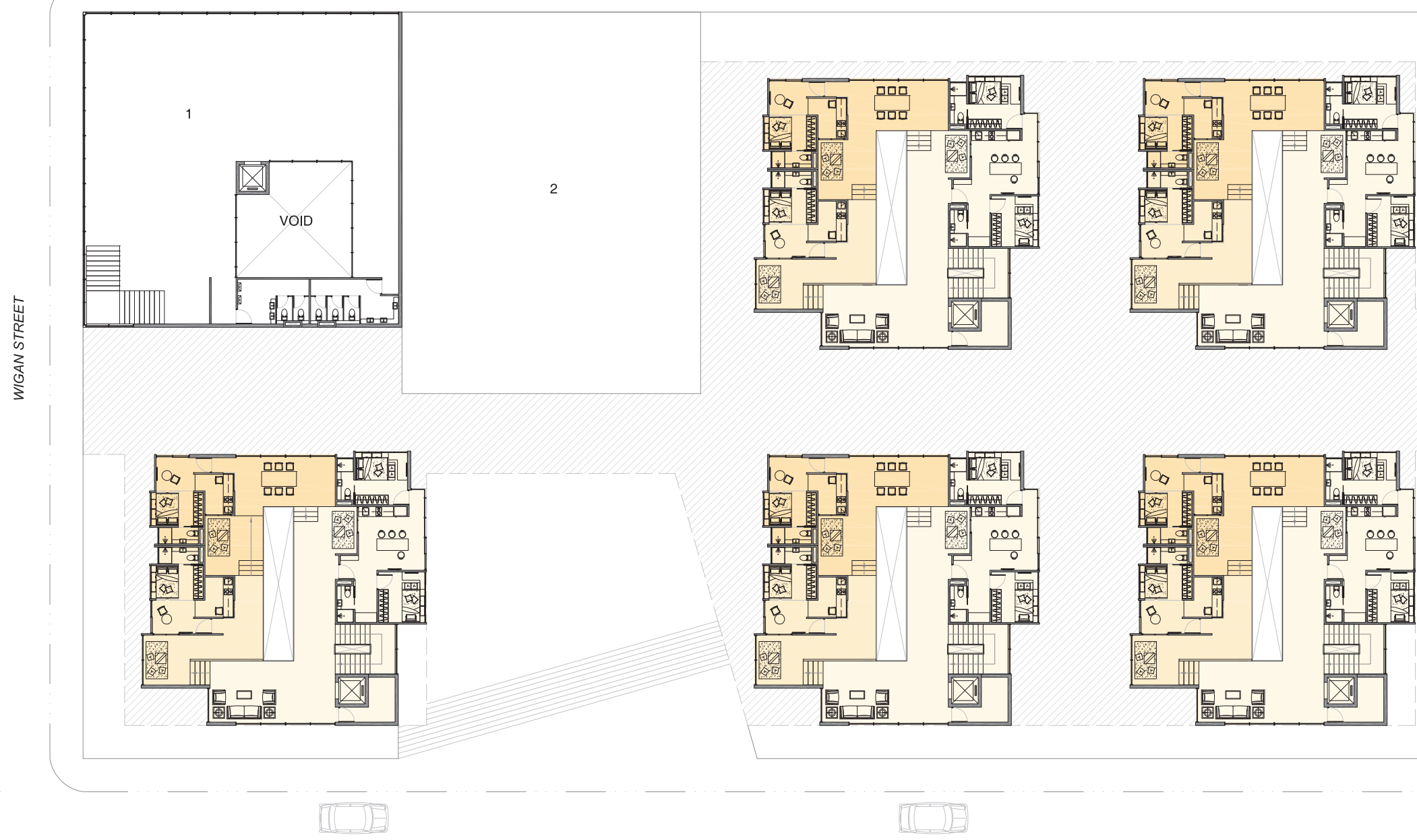

CANTILEVERED

LIGHTHOUSE CINEMA ROOF TERRACE 


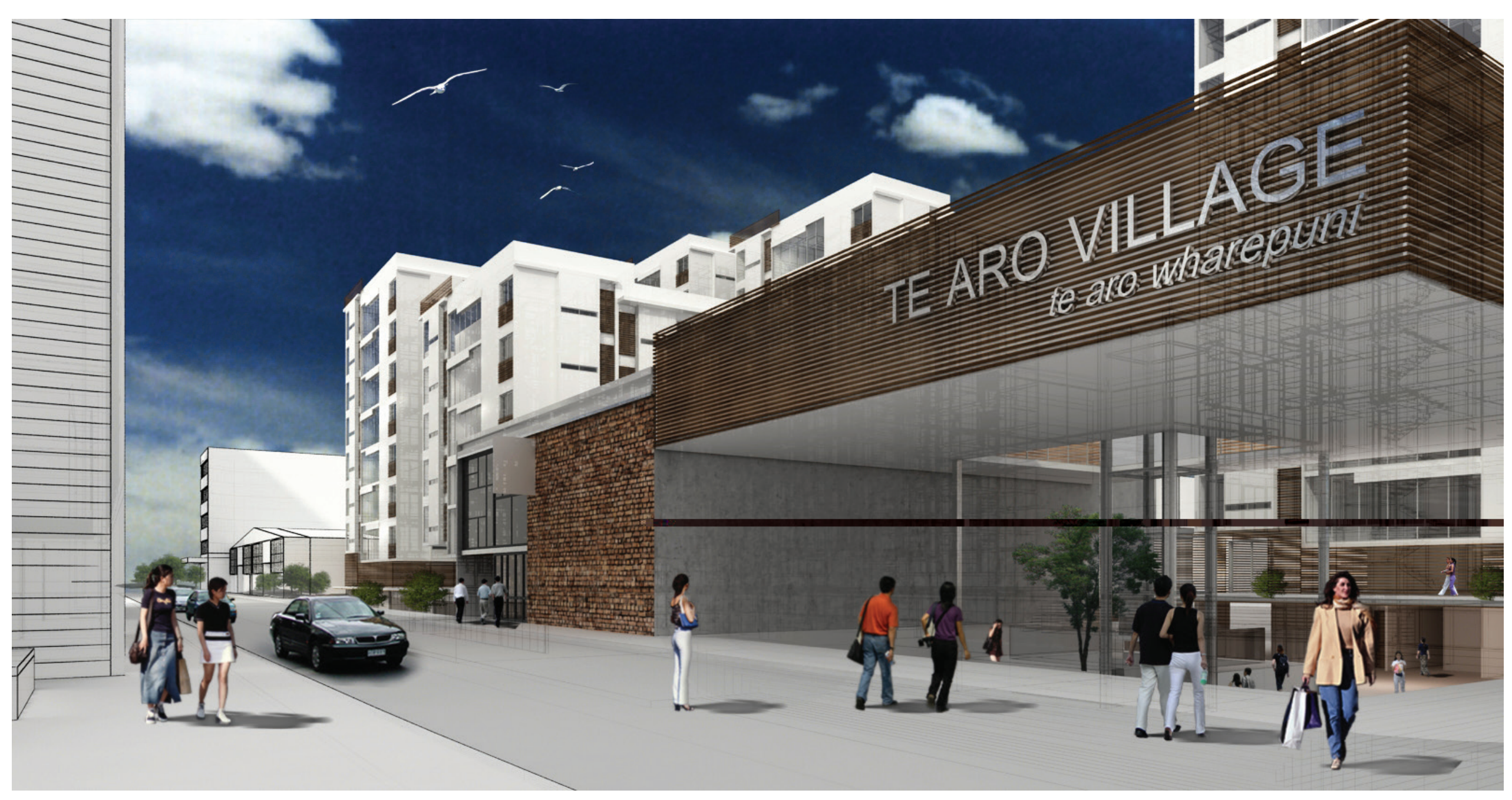


4 Figure 61. Entrance from Wigan Street corner through the exhibition space

- Figure 62. Entrance from Abel Smith Street through the public park behind Light House Cinema

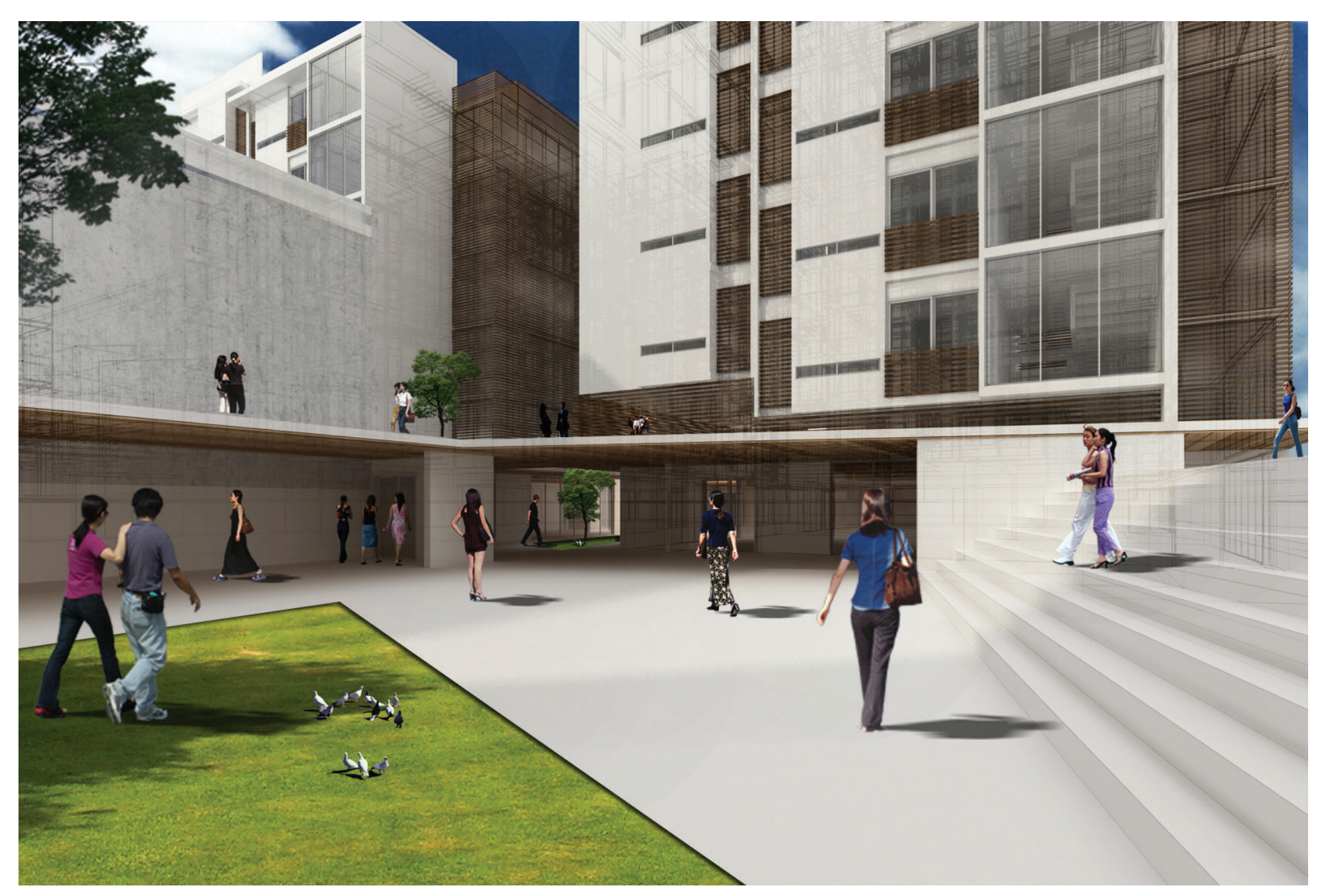




\section{- Figure 63.}

Longitudinal street sections indicating the relationship

between Te Aro Village and

the university campus as well as southern cross apartments

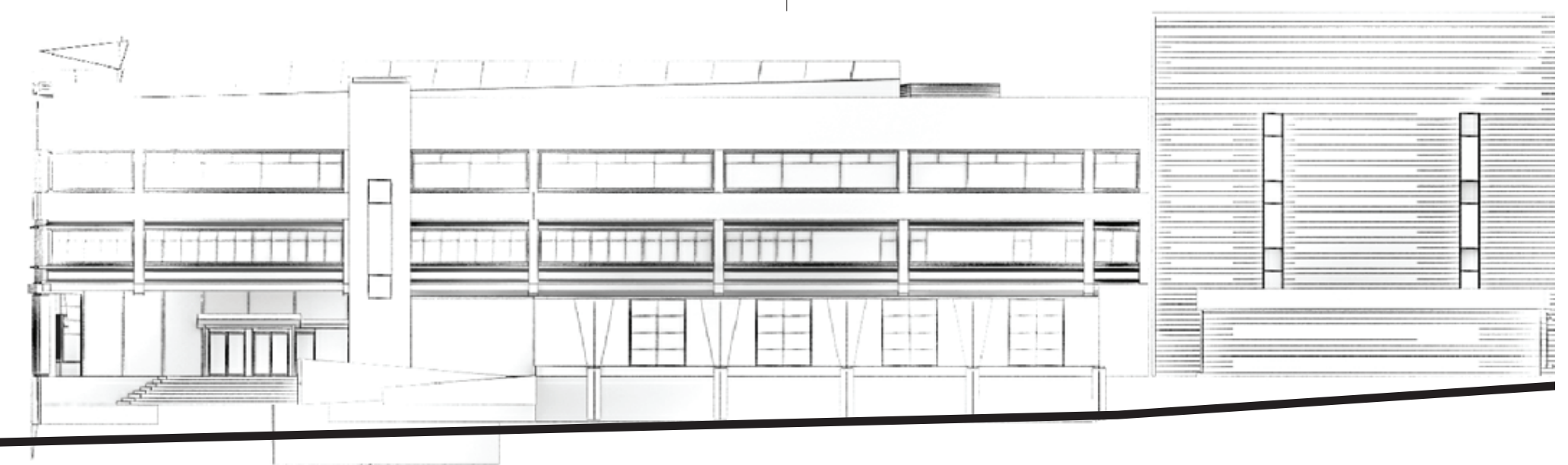




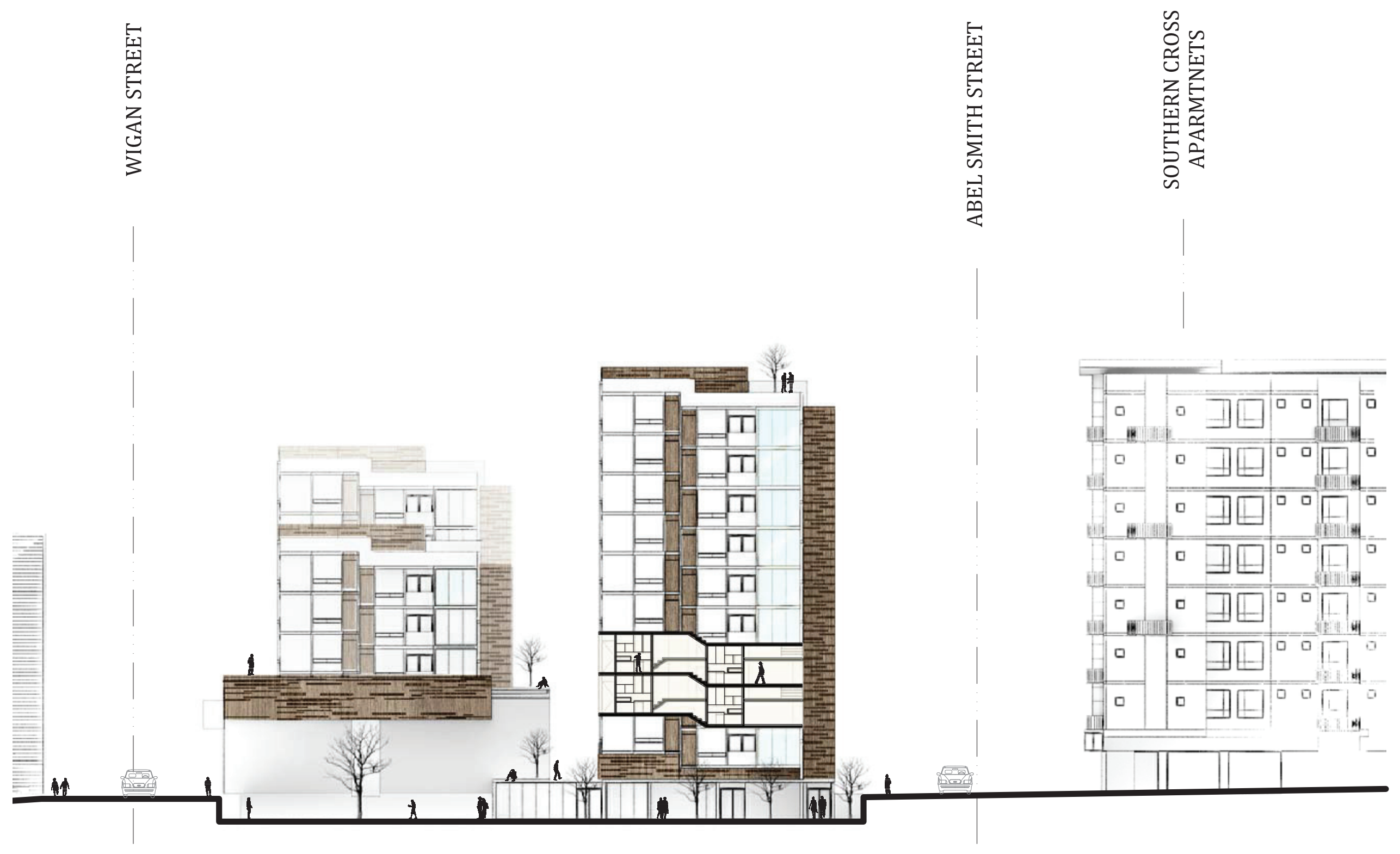


In terms of the apartments' internal layout, the idea from the previous shared house proposal of having split level circulation carried all the way to the inhabitable roof tops spirally, and defined transitional shared space for each individual unit was retained. An internal void in the middle enhanced the visual interactions through multiple levels while utilising the shared circulation space. Numbers of unit types were simplified and reduced from three to two in this proposal. The previous single studio was removed due to the extent of its limitation on interior functional spaces. The remaining two types were double studios and two bedroom units. The interior layout of the double studio remained almost the same as previous, but the new two bedroom unit has been enlarged by having separated toilet facilities for each bedroom. Separate private facilities in the two bedroom unit prevented the unnecessary disturbance between the two residents and made it function as two attached single studios. 
The perspectives of the double studio rooms demonstrate that the interior space is elegantly divided according to its functional use. Unlike local examples entering from a small corridor with a kitchen, a full height glass sliding door was placed directly facing the unit entrance, which turning the entrance space into a balcony or a loggia. Such outdoor connected experience of entering created a distinct openness in this compact living situation. The bedroom area was designed to be kept away from the main circulation in order to maintain necessary privacy and receive more sun light. A full height timber bi-fold door in the kitchen could be opened up and lead to the transitional share space out in the main circulation area. 


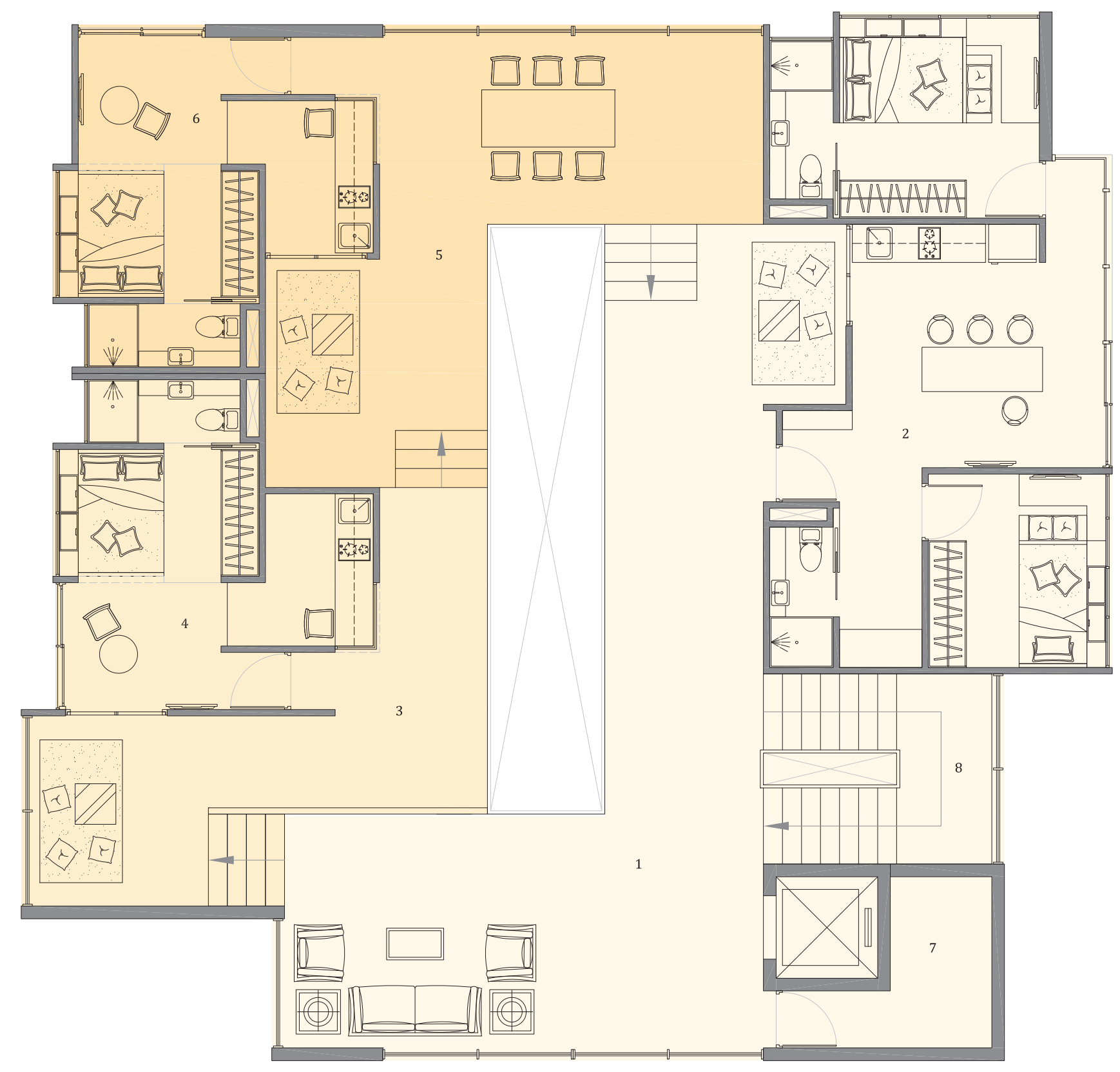

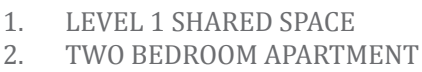

4. DOUBLE STUDIO A

5. LEVEL 1B SHARED SPACE

5. DOUBLE STUDIO B

6. DOUBLE STUDIO
7. UTILITY ROOM

8. EMERGENCY STAIRCASE 
4 Figure 64. Enlarged Te Aro Village apartment floor plan, indicating the split

level circulation and the transitional shared space created within it

$\boldsymbol{\nabla}$ Figure 65. An internal perspective view from outside the elevator to the

north, overlooking the spatial quality of multiple split level circulation.

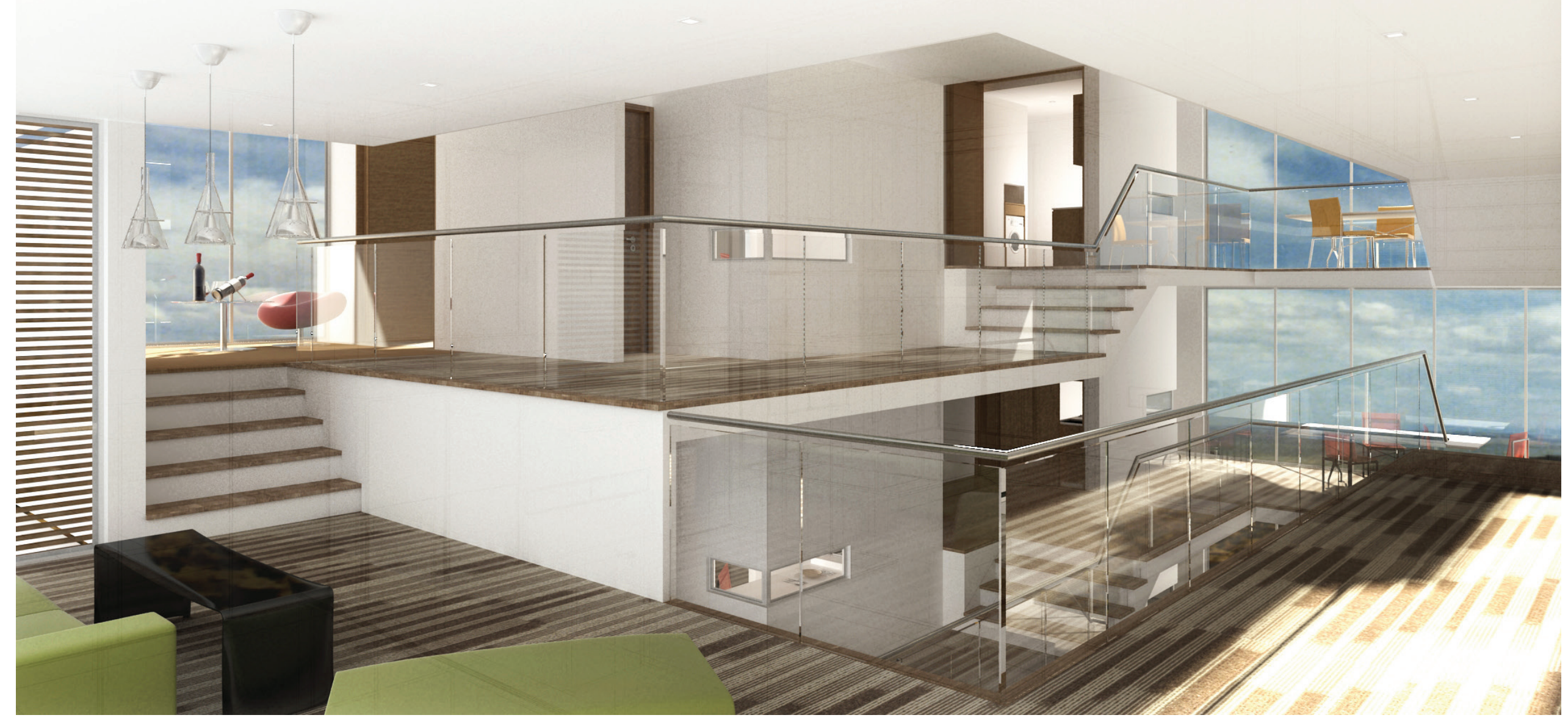




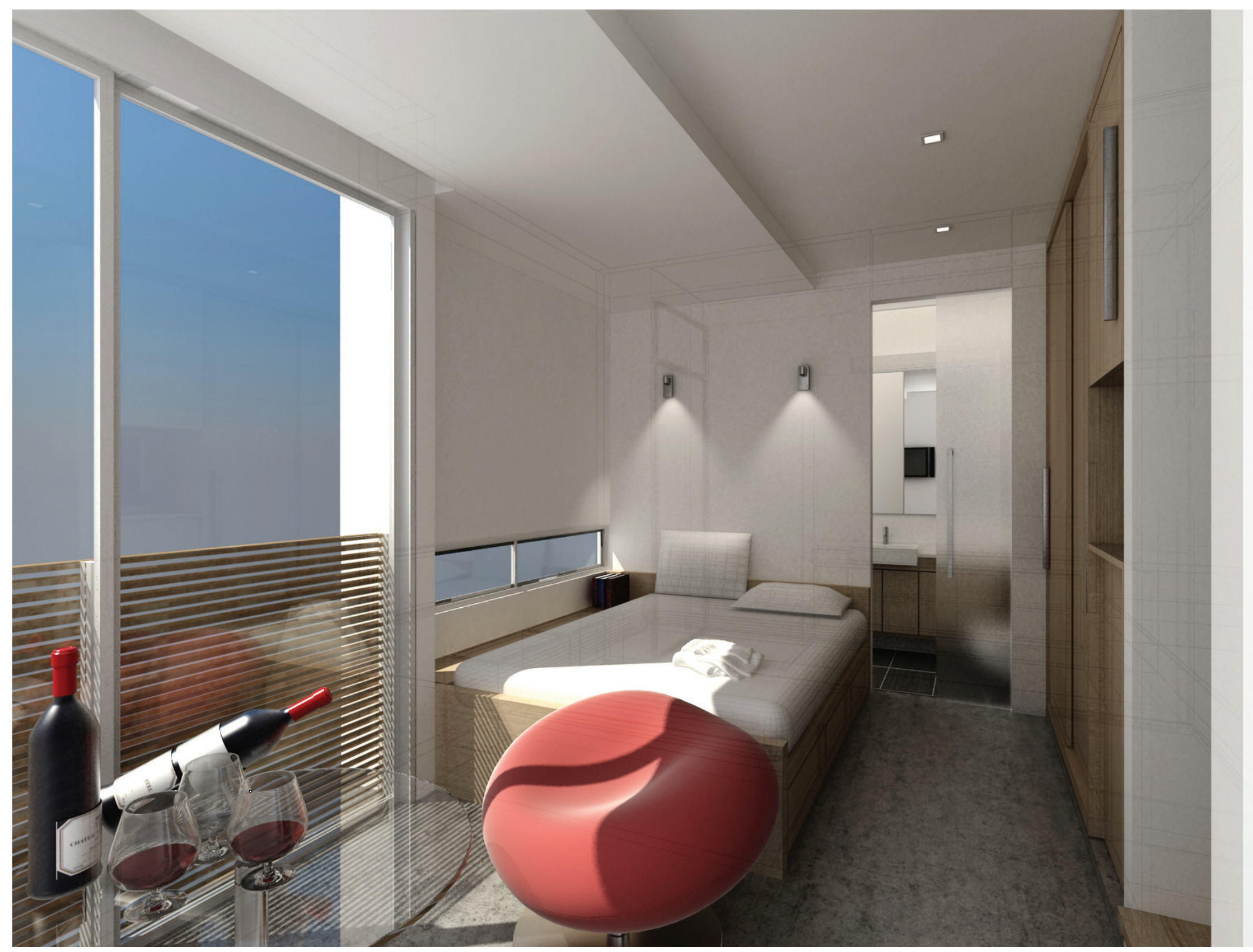




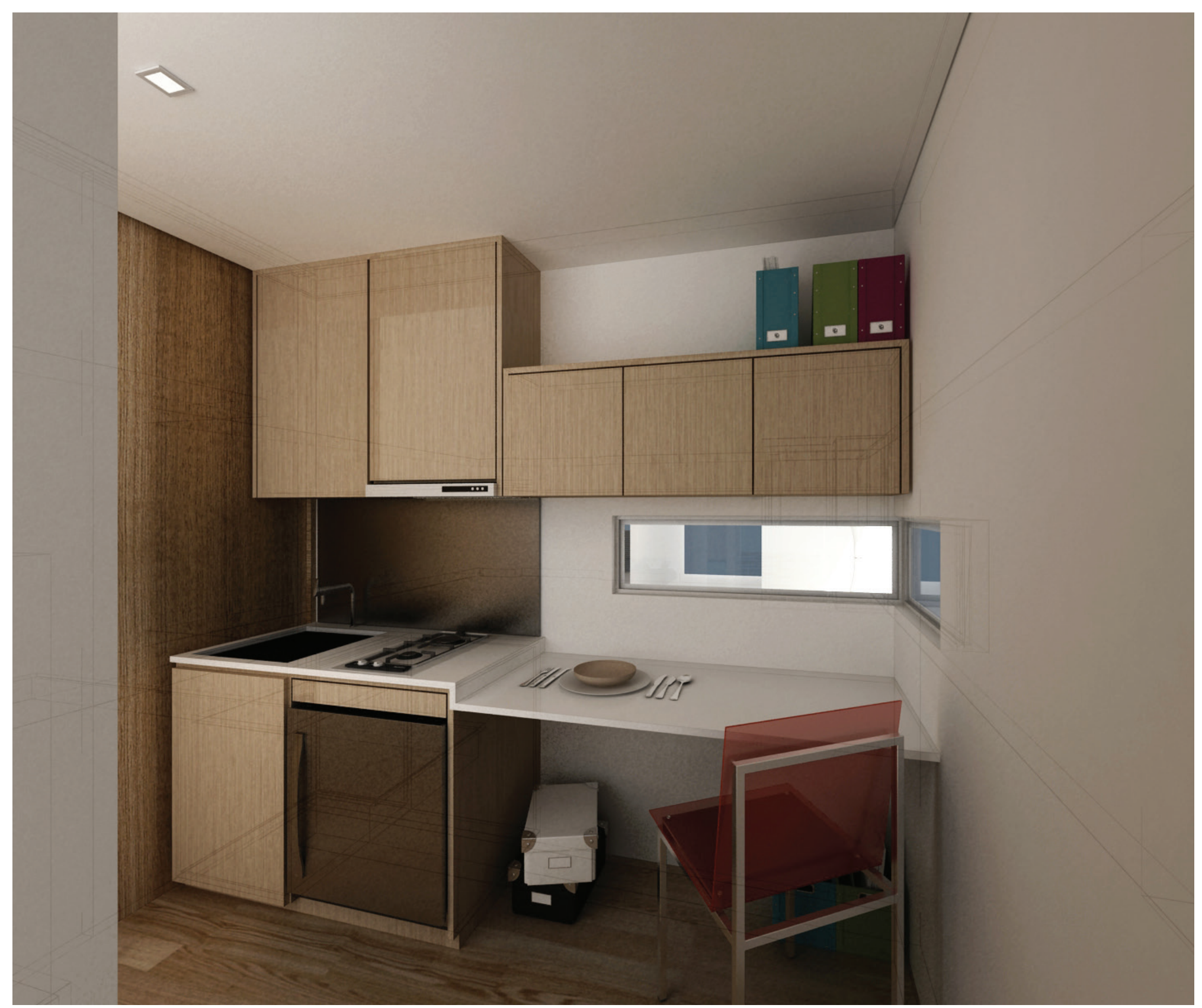


There were 38 two bedroom apartments and 76 double studios contained in the five apartment towers in total, resulting in an overall site density of approximately 410 habitable rooms per hectare. In comparison to the previous shared house proposal, the density in Te Aro Village has been reduced because the public areas and Light House Cinema are part of the calculation. If the residential density in the five apartment towers only is considered, it would increase to approximately 1010 habitable rooms per hectare; which was the highest result among all the previous design iterations. So the design balances high density and high amenity. Te-puni Village as case study shown in the early chapter had achieved approximately 840 bedrooms per hectare by having mostly single studio units with shared toilet and bathroom facilities. In comparison to Te-puni, the proposed Te Aro Village not only achieved a higher density, but also a better quality living environment by having significant individual private facilities in all units, transitional shared space adjacent to units, and easy access to a range of shared common facilities. 


\subsection{FINDINGS AND REFLECTIONS}

After investigating individual units and single tower apartments, the final design scheme had looked at sharedness on a larger scale of university campus housing. The balance in between residents and pedestrians, private and public space, openness and enclosure as well as living density and leisure facilities have been tested in a wider context. Instead of drawing a fine line to isolate the private territory, the proposal argued and demonstrated that hierarchy of floor levels could allow the private and public space to co-exist both visually and physically. A more friendly, inhabitable, space with interactions between private and public was created by blurring the boundaries. Apart from having an efficient internal layout, the quality of life in medium or higher density compact living environments could also be improved by extending the internal shareness concept to the external. The overlapping territories with blurred boundaries enhanced the conversation between the private community and the general public, which gave diverse options for students who lived in this village to experience the surrounding environment and make connections with the outside world.
In terms of social issues, responsibility of the maintenance to the public and shared space was quite a common concern in such a shared design scheme. Similar to other urban residential communities, the common spaces are usually maintained by a body corporate structure, which in this case would be taken care of by the University who operates this campus village. 


\section{CONCLUSION}

The three proposed design schemes presented in this thesis have addressed the question of how to improve quality of life in medium or higher density student housing at different scales respectively. They addressed the critiques of current local student living environments, and gave solutions to achieve better living quality from both internal and external aspects.

The first investigation looked at how a limited space can be maximised and expanded in order to be felt to be larger than what it actually is. Strategies of hyper efficiency, transformative and multi-purposed functional areas were explored. The proposed design of compact single units demonstrated that spatial quality can be improved by allowing occupants to have the chance to explore the internal defined space through split levels. It also allows greater volume within a smaller plan area. The two-bedroom unit design extended the investigation and introduced the idea of privacy sharing within a compact unit design. By having separated common areas, the expanded space was not only able to hold larger occasional functions, but also retained the necessary privacy which allowed it to be used by different occupants individually.

The second stage of the thesis shifted emphasis from individual housing to apartment block design, in order to test and explore how hyper efficiency could be applied to common areas of medium and higher density living environments. The first iteration, a single unit complex, started to deal with community living and began to provide quality external linkages between the units and blocks. It also explored public functions and shared space. The experimental result turned out to be a composite building with good quality external public spaces, but with underutilised internal corridors. Planning of internal circulation could potentially make a difference to improve the spatial quality and density of the living experience. The shared house typology was then studied and influenced the next phase of the design. The idea was to create internal functional circulation that was able to be shared by residents. Instead of expanding the floor area of individual apartments, transitional platforms were designed between each unit and the main internal circulation to create a spatial richness. Such transitional, in-between spaces function as part of the main circulation yet have the sense of individual allocated space that can be occupied 
when needed to accommodate guests or to enable to occupants some social engagement.

The final design phase explored the shared space idea within a wider context. It investigated the third space in between private and general public territories by introducing a degree of sharedness into private residential community in a larger site. The proposed recessed boundary transformed the sharing concept externally, and improved the living quality by creating diverse gradients of visual and physical sharing, and facilitating potential interactions between the residents and the public. Reflecting on this proposal within the larger group of apartments, the challenge was to get the right balance of external shared space. A variation of private residential community that suited the local situation was then developed. The balance in this proposal was achieved by forming diverse interacting layers of circulation spaces that could be occupied by residents and public pedestrians respectively. Improved quality of life is achieved through preventing social isolation, with overlapping territories, blurred boundaries, and diverse on-site shared leisure facilities offered.

The design iterations scale from individual housing to a large student village. The result suggests that the quality of life in medium and higher density student housing can be definitely improved through hyper efficient and shared space design. Shared space was introduced in the discussion of this thesis by creating a transitional in between space as a part of the main circulation; yet retaining a sense of territory that allows the private space to be opened and expanded for occasional needs. Such a shared house typology changes the way of thinking in conventional apartment living, where the common space and circulation are restricted and limited by narrow corridors. Instead, having a series of in between space would transform the conventional circulation into a quality, spacious and multi-functional shared area with much more visual richness and living options. The proposed designs suggest a new way of student living in a higher density built environment in the inner city of Wellington. Better quality of life in such compact living environments can be achieved with hyper efficiency, shared space and blurred boundaries. 


\section{BIBLIOGRAPHY}

Andrea, H. (2012). Small Moves: architectural strategies for the unit in social housing. In Soules, M. (Eds.), Future Social. Vancouver, Canada: Blueimprint

Dorgan, K. \& Evans, D. (2008). Mainstreaming Good Design in Affordable Housing. In Bell, B. \& Wakeford, K. (Eds.), Expanding Architecture: Design as Activism. New York, US: Metropolis Books.

El Nasser, H. (2002, December 15). Gated communities more popular, and not just for the rich. USA TODAY. Retrieved from: http://usatoday30. usatoday.com/news/nation/2002-12-15-gated-usat_x.htm

Goodchild, B. (1997). Housing and the Urban Environment, A guide to housing design, renewal and urban planning. Oxford: Blackwell Science Ltd.

Neuman Faia, J. D., \& Ap, L. (2013). Building type basics for College and University Facilities (2nd ed.) US: Wiley.
Özsoy, A., \& GÖkmen, G. (2005). Space Use, Dwelling Layout and Housing Quality: An Example of Low-Cost Housing in Istanbul. In R. G. Mira, D. L. Uzzell, J. E. Real \& J. Romay (Eds.), Housing, Space and Quality of Life (pp. 1728). Hants, England: Ashgate.

Property problems: Gated communities surprise new migrants. (2004, February 7). The New Zealand Herald. Retrieved from: http://www. nzherald.co.nz/business/news/article.cfm?c_id=3\&objectid=3547542

Richardson, P. (2011). Nano House. New York, US: Thames \& Hudson Inc.

Small home, big picture. (2009). Cube project QB2. Retrieved from: http:// www.cubeproject.org.uk/

Stwart, M. (2004). In Koolhass, R., \& McGetrick, B. (Eds.), Content: triumph of realization. (pp. 224). Köln, Germany: Taschen. 
Thorne, R. (1992). ANOTHER SIDE TO BEING A CLEVER COUNTRY: Working and Housing Environment for University Students. Sydney, Australia: University of Sydney.

Wellington City Council. Central Area Building Heights. Retrieved from: http://wellington.govt.nz/ /media/your-council/plans-policies-andbylaws/district-plan/volume03/files/v3map32.pdf 
